\title{
NEUROCOGNITIVE AND DYSFUNCTIONAL BELIEF CANDIDATE ENDOPHENOTYPES OF OBSESSIVE-COMPULSIVE AND \\ RELATED DISORDERS
}

\author{
by
}

Stephanie E. Taillefer

Master of Arts in Psychology, Ryerson University, 2012

Bachelor of Science in Psychology, Queen's University, 2009

A dissertation presented to Ryerson University

In partial fulfillment of the requirements for the degree of

Doctor of Philosophy in the Program of Psychology

Toronto, Ontario, Canada, 2019

(C) Stephanie E. Taillefer 2019 


\section{Author's Declaration Page}

I hereby declare that I am the sole author of this dissertation. This is a true copy of the dissertation, including any required final revisions, as accepted by my examiners.

I authorize Ryerson University to lend this dissertation to other institutions or individuals for the purpose of scholarly research

I further authorize Ryerson University to reproduce this dissertation by photocopying or by other means, in total or in part, at the request of other institutions or individuals for the purpose of scholarly research.

I understand that my dissertation may be made electronically available to the public. 


\begin{abstract}
Neurocognitive and Dysfunctional Belief Candidate Endophenotypes of Obsessive-Compulsive and Related Disorders

Stephanie E. Taillefer

Doctor of Philosophy in the Program of Psychology, 2019

Ryerson University

Background: The current dissertation examined neurocognitive and dysfunctional belief candidate endophenotypes (CEs) across the obsessive compulsive spectrum to elucidate general versus specific factors. This study included CEs from two etiological perspectives well established in the literature. Secondary analyses examined several CEs multidimensionally and examined the relationship between CEs and of QOL. Methods: A total of 77 participants took part in this study, divided into four groups; OCD $(n=21)$, Hoarding Disorder (HD; $n=16)$, Grooming Disorders which included both Trichotillomania and Excoriation Disorder (GD; $n=$ $18)$, and control participants $(n=22)$. Participants completed a clinical interview and battery of neurocognitive tasks and questionnaires. Results: Those with HD performed worse than controls on measures of response inhibition and set-shifting. OCD continued to predict significant variance in number sequencing. Examination of dysfunctional belief CEs revealed specificity of Responsibility/Threat beliefs and Importance/Control of Thoughts beliefs to OCD. Perfectionism/Intolerance of Uncertainty appear to be broad CEs; however, differing specificity emerged depending on the measure utilized to measure the construct. Self-report indecision revealed specificity to OCD and HD. Differing patterns of QOL impairments emerged across the spectrum. A better understanding of CEs specificity has implications for diagnostic classification, etiology, course, and treatment.
\end{abstract}




\section{Acknowledgements}

First and foremost, I would like to thank my supervisor, Dr. Kristin Vickers for your unwavering support and mentorship throughout this process. In addition to your skill and wisdom, I appreciate your patience, kindness, helpfulness and openness. These qualities make you an outstanding supervisor, and I feel lucky to have had you at the helm throughout this journey.

I would also like to thank the members of my dissertation committee, Dr. Margaret (Peggy) Richter and Dr. Tisha Ornstein. Peggy, you are the most talented clinician I have had the privilege to learn from, and this clinical skill and understanding allows you to formulate cuttingedge research questions that improve the quality of life for so many of your patients. Thank you for taking me under your wing. My experience with you at Sunnybrook added a level of richness and purpose to my graduate school experience that I am forever grateful for. Tisha, I am grateful for your wisdom and guidance, and am thankful to you for welcoming me into your research space. I would also like to thank you for your insightful questions and suggestions. I have learned so much from you and I so appreciate your research acumen as it guided my research. I would also like to thank my external reviewer, Dr. Steven Taylor, for his careful and insightful review of the dissertation. His suggestions assisted with conceptualization and, ultimately, produced a far superior document.

To my family and friends who have supported me over these seven years of postsecondary education, thank you for your patience and support. I would like to thank Jeanine Lane for her help with this project and her unwavering support for me as a colleague and friend. Your time in the trenches with me is appreciated! To Duncan, thank you for being my rock, 
particularly over these final few years. To my mother and father, thank you for instilling a love of education and pursuit of knowledge. Thank you for always being a phone call away. 


\section{Dedication}

I dedicate this dissertation to Torch, my black and white soul mate, who lay at my feet for the entire eight year process. 


\section{Table of Contents}

Author's Declaration Page................................................. ii

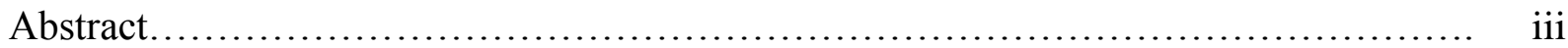

Acknowledgements..................................................... iv

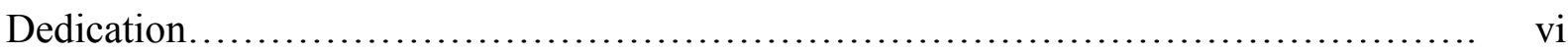

Table of Contents............................................................ vii

List of Tables.........................................................

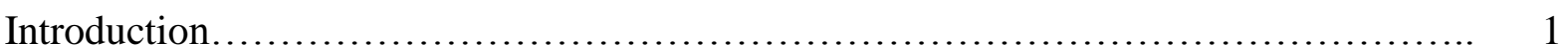

Introduction to the OCRDs................................................... 2

Comorbidity....................................................... 7

First-line Treatment of the OCRDs..................................... 9

Neurobiology of OCRDs.............................................. 11

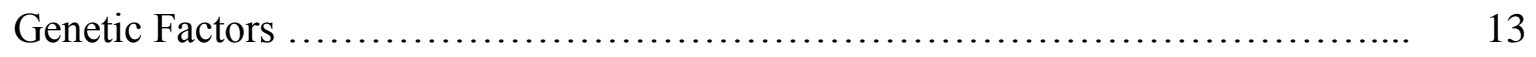

Endophenotypes.................................................... 17

Candidate Endophenotypes of OCD .................................... 19

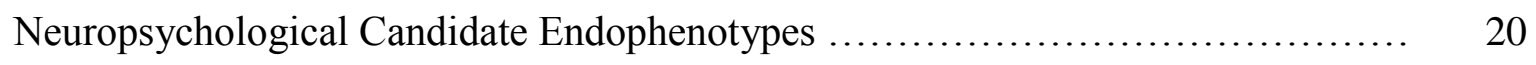

Inhibitory Dysfunction.......................................... 22 
Cognitive Flexibility.

Memory

Decision Making.

Dysfunctional Beliefs as Candidate Endophenotypes

Impulsivity.

Neurocognition, Functional Impairment, and Quality of Life.

Current Study.

Clinical Implications.

Method.

Participants.

Power.

Measures

Procedure.

Statistical Analysis.

70

Results

77

Demographic and Clinical Characteristics. 
Neurocognitive Results.

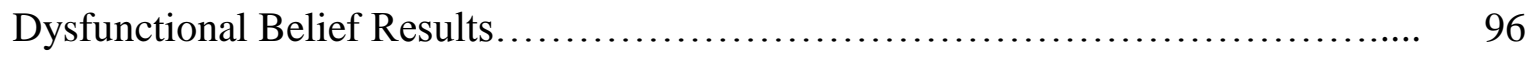

Associated Construct Results.................................................... 104

Quality of Life Results.................................................... 107

Results Summary of Significant Findings..................................... 114

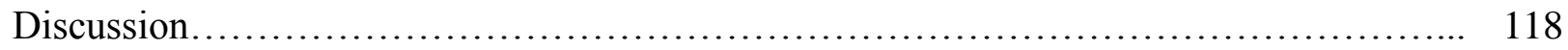

Interpretation of Results.............................................. 120

Limitations............................................................ 131

Broader Implications ............................................................ 137

Future Directions.......................................................... 138

Conclusions................................................................... 141

Reference List................................................................... 142 


\section{List of Tables}

Table 1 Sociodemographic characteristics of the sample......................... 79

Table 2 Clinical characteristics of the sample................................. 82

Table 3 Performance on neurocognitive measures of patients with obsessivecompulsive disorder (OCD), hoarding disorder (HD), a grooming disorder

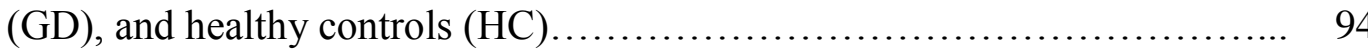

Table 4 Dysfunctional belief measures of patients with obsessive-compulsive disorder (OCD), hoarding disorder (HD), a grooming disorder (GD), and healthy controls

$(\mathrm{HC})$

Table 5 Associated construct measures of patients with obsessive-compulsive disorder (OCD), hoarding disorder (HD), a grooming disorder (GD), and healthy controls $(\mathrm{HC})$

Table 6 Quality of life measures of patients with obsessive-compulsive disorder (OCD), hoarding disorder (HD), a grooming disorder (GD), and healthy controls (HC)

Table 7 Relationship between candidate endophenotypes and quality of life expressed as Pearson's correlations. 


\section{Introduction}

The Diagnostic and Statistical Manual of Mental Disorders Fifth Edition (DSM-5; American Psychiatric Association, 2013) recently reclassified Obsessive-Compulsive Disorder (OCD) into its own spectrum of disorders called Obsessive-Compulsive and Related Disorders (OCRDs). This change is a substantial shift from its previous designation as an anxiety disorder in earlier versions of the DSM. OCRDs include OCD, Hoarding Disorder (HD), Trichotillomania (hair-pulling; TTM), Excoriation (skin picking) Disorder (ED), Body Dysmorphic Disorder (BDD), Substance/Medication-Induced OCRD, OCRD due to another medical condition, and other specified and unspecified OCRDs. This shift in conceptualization was an attempt to group disorders together that were characterized by repetitive thoughts and/or behaviour (American Psychiatric Association, 2013). Although there is not a consensus on the conceptual or empirical grounds for this shift (e.g., Storch, Abramowitz, \& Goodman, 2008), the change reflects increasing evidence that these disorders more closely share phenomenology, comorbidity, treatment response, and neurobiology (e.g., genetic factors, neurochemistry) than do other anxiety or impulse control disorders (e.g., Stein et al., 2010; Zohar, 2006).

Endophenotypes are considered vulnerability traits, defined as intermediate phenotypes linking "the pathway between disease (phenotype) and distal genotype" (Gottesman \& Gould, 2003, p. 636), and provide a way to examine the etiology of complex psychiatric disorders (Taylor, 2012). Neurocognitive functioning and, more recently, dysfunctional beliefs, have been posited to be candidate endophenotypes (CEs) in OCD. The overarching goal of the current dissertation is to examine neurocognitive functioning and dysfunctional beliefs across the OCRDs to elucidate general versus specific factors associated with disorder. Previous work is reviewed, and recommendations made in critical reviews were incorporated, where possible, into 
the current research design. In an exploratory fashion, this dissertation also examines $\mathrm{CE}$ predictors of QOL and examines several multidimensional constructs (e.g., perfectionism, intolerance of uncertainty (IU), impulsivity) using multiple measures. The recommendation of administering multiple tests within a study to measure a multidimensional construct has been highlighted by several reviews (Abramovitch, Mittelman. Tankersley, Abramowitz, \& Schweiger, 2015; Lezak, Howieson, Bigler, \& Tranel, 2012).

\section{Introduction to the OCRDs}

OCD affects approximately 2-3\% of the population (Kessler et al., 2005, Weissman et al., 1994). It is defined by the presence of obsessions (repetitive thoughts/images/impulses that are unwanted, intrusive and distressing), and/or compulsions (repetitive behaviours or mental acts) that are time consuming and result in significant distress or impairment. Common examples of obsessions include thoughts about contamination or thoughts about symmetry, while common compulsions include hand washing, counting, or arranging. The average age of onset of symptoms shows a bimodal distribution (10 years and early adulthood), with males being more likely to develop OCD during childhood/adolescence and females developing OCD more frequently in early adulthood (Dell'Osso et al., 2016; Grant, Chamberlain, \& Odlaug, 2014). The gender distribution is approximately equal in males and females, and the typical untreated course is that of chronic waxing and waning symptoms that often persist into middle-age (Grant et al., 2014). OCD is associated with significant impairment and decreased work attendance and productivity (e.g., Ruscio, Stein, Chiu, \& Kessler, 2010; Sorensen, Kirkeby, \& Thomsen, 2004). While economic cost estimates of OCD in Canada specifically are limited, a study in Europe in 2012 estimated that OCD costs 2272 million euro (approximately 3.6 billion Canadian dollars) in direct and indirect costs (Olesen, Gustavsson, Svensson, Wittchen, \& Jonsson, 2012). In the 
United States, estimates indicate that approximately 10.6 billion dollars (USD) are spent annually on OCD treatment alone (Eaton et al., 2008). While more recent estimates are difficult to find, it is likely that both of these figures are an underestimate in 2018 due to the cost of inflation.

HD is defined by persistent difficulty discarding or parting with possessions due to a perceived need to save the items and distress associated with discarding them, resulting in living spaces that are cluttered to the point where the room can no longer be used for its intended purpose (American Psychiatric Association, 2013). DSM-5 includes an acquisition specifier, to indicate whether excessive acquisition is currently present through compulsive buying or the acquisition of free things. The average median age of symptom onset is between $10-20$ years; however, the average median age of a diagnosis was between 20 and 30 years (Dozier, Porter, \& Ayers, 2015). For approximately $25 \%$ of individuals, they reported later onset, after the age of 40 (Dozier et al., 2015). It affects slightly more males than females, and the course of illness is chronic without treatment, with symptoms increasing in severity over time (Dozier et al., 2015; Grant et al., 2014).

The classification of hoarding behaviour has evolved over time. DSM-IV-TR listed hoarding behaviour as one of eight criteria for obsessive-compulsive personality disorder (OCPD). This conceptualization dates back to the psychoanalytic concept of anal personality (Freud, 1908), originally described as orderliness, obstinacy, and parsimony. While hoarding does not appear in DSM-IV-TR as a symptom of OCD, examination of the differential diagnosis section in DSM-IV-TR between OCPD and OCD states '...A diagnosis of OCD should be considered especially when hoarding is extreme (e.g., accumulated stacks of worthless objects present a fire hazard and make it difficult for others to walk through the house' (p. 728). 
Additionally, hoarding behaviour is included as a symptom in many of the gold-standard OCD structured interviews and questionnaires (e.g., Yale-Brown Obsessive-Compulsive Scale, Goodman et al., 1989; Obsessive-Compulsive Inventory-Revised, Foa et al., 2002). Thus, the inconsistency and ambiguity of the classification of hoarding in DSM-IV-TR has led some to conceptualize hoarding as a symptom of OCD (e.g., Ong, Pang, Sagayadevan, Chong, \& Subramiam, 2015; Van Ameringen, Patterson, \& Simpson, 2014), while others have conceptualized hoarding as a characterological trait best captured in OCPD (Abraham, 1921; Freud, 1908; Jones, 1918). For a review of the classification of hoarding disorder, see MataixCols et al. (2010).

Recent research, however, suggests that hoarding is best understood as a distinct disorder, leading to its reclassification in DSM-5. Specifically, studies suggest that more than $80 \%$ of individuals with HD do not meet diagnostic criteria for OCD (Mataix-Cols et al., 2010), which corroborates cluster and factor analysis studies indicating that OCD and HD load on to different factors (Bloch, Landeros-Weisenberger, Rosario, Pittenger, \& Leckman, 2008). The North American lifetime prevalence rate of HD ranges from 2- 5\% (Ruscio et al., 2010; Samuels et al., 2008). HD is associated with family, work, and social disability, in addition to safety concerns including fires, falls, poor sanitation, and health risks from clutter (Damecour \& Charron, 1998; Steketee, Frost, \& Kim, 2001). Motivation for change in hoarding disorder is problematic and thought to be lower than in related disorders, primarily due to the ego-syntonic nature of hoarding symptoms and associated lack of insight (Christensen \& Greist, 2001; Steketee, Frost, Wincze, Greene, \& Douglass, 2000; Steketee et al., 2001).

TTM is characterized by repetitive hair pulling, resulting in noticeable hair loss, coupled with repeated attempts to decrease or stop pulling, and clinically significant distress or role 
impairment (American Psychiatric Association, 2013). This disorder was previously classified as an impulse control disorder not otherwise specified, prior to being incorporated into the OCRDs in DSM-5. TTM has a lifetime prevalence rate of approximately 0.6-3\% (Christenson, Pyle, \& Mitchell, 1991; Grant, Levine, Kim, \& Potenza, 2005). Rituals around the pulled hair are common, and include examining, manipulating, biting, chewing, or swallowing the hair (Christenson et al., 1991; Mansueto, 1991). The functional impact of the disorder can be severe, with individuals reporting interference in home management and ability to maintain close relationships, leading to avoidance of haircuts, sports, sexual intimacy, or public activities more broadly (Sternberger, Thomas, Mansueto, \& Carter, 2000; Woods et al., 2006).

ED is characterized by repetitive skin picking resulting in tissue damage, repeated unsuccessful attempts to decrease or stop skin picking, and clinically significant distress or impairment (American Psychiatric Association, 2013). This disorder was historically classified as an impulse control disorder, not elsewhere classified prior to DSM-5. It has a lifetime prevalence rate of approximately 1.4-5.4\% (Hayes, Storch, \& Berlanga, 2009; Keuthen, Koran, Aboujaoude, Large, \& Serpe, 2010). Picking sites on the body can be varied, with the face cited as the most common site. Other frequent picking sites include fingers, torso, arms, legs, back, pubic area, and feet (Bloch, Elliott, Thompson, \& Koran, 2001; Flessner \& Woods, 2006; Grant, Odlaug, \& Kim, 2007; Odlaug \& Grant, 2008a; Wilhelm, Otto, Lohr, \& Deckersbach, 1999). ED often results in significant scarring and infections (Arnold et al., 1998; Gupta, Gupta, \& Haberman, 1986; Odlaug \& Grant, 2008a, b), which, when most severe, can be life threatening (O’Sullivan, Phillips, Keuthen, \& Wilhelm, 1999).

TTM and ED have both been conceptualized as body-focused repetitive behaviours resulting in distress and impairment. In both disorders, research suggests that pulling or picking 
serves to regulate affective states and arousal (Neziroglu, Rabinowitz, \& Breytman, 2008).

Research has documented that many individuals with TTM and ED experience short term relief or pleasure as a result of picking (Bloch et al., 2001). Often, impulses or urges precede the pulling or picking, which can be in response to external cues (e.g., bedroom, mirrors) or internal cues (e.g., anxiety, thoughts). Due to their phenomenological similarities (e.g., Lochner, Simeon, Niehaus, \& Stein, 2002; Odlaug \& Grant, 2008a, b), and the findings that TTM and ED have more similarities with each other than other OCRDs (e.g., Snorrason, Belleau, \& Woods, 2012), studies have routinely collapsed the TTM and ED groups into a general GD group for analysis (Flessner, Woods, Franklin, Keuthen, \& Piacentini, 2009; Grant \& Christenson, 2007; Hanna, Fischer, Chadha, Himle, \& Van Etten, 2005), as will occur in the current dissertation. Support for this grouping comes from the first twin study to include all of the OCRDs in the same analyses (Monzani, Rijsdijk, Harris, \& Mataix-Cols, 2014); in this work, one factor emerged that was exclusively related to TTM and ED, suggesting that these disorders may, in fact, be different phenotypic expressions of the same disorder (Monzani et al., 2014). In terms of age of onset, gender, and course, the average age of onset of symptoms in the grooming disorders is 12-13 years (Grant et al., 2014). The gender distribution suggests females are more likely to be affected, and the course waxes and wanes into adulthood (Grant et al., 2014).

$\mathrm{BDD}$ is characterized by an intrusive preoccupation with imagined or exaggerated imperfections in appearance that result in significant distress and impairment in functioning (American Psychiatric Association, 2013). BDD is also associated with behavioural rituals, aimed at examining, improving, or hiding the perceived flaw, such as mirror checking, reassurance seeking, or elaborate makeup routines (Phillips, Menard, Fay, \& Weisberg, 2005). DSM-5 includes a muscle dysmorphia specifier, which reflects a presentation in which the 
individual is excessively concerned with being muscular. BDD was historically conceptualized as a Somatoform Disorder, ${ }^{1}$ but was reclassified as an OCRD in DSM-5. BDD is associated with avoidance of seeking treatment, high levels of suicidality with lifetime suicide attempts ranging from 24-28\%, social isolation, and high levels of psychiatric hospitalization (Buhlmann et al., 2010; Conroy et al., 2008; Perugi et al., 1997; Phillips, McElroy, Keck, Pope, \& Hudson, 1993; Phillips et al., 2005; Phillips \& Diaz, 1997; Phillips \& Menard, 2006; Veale, Boocock, Gournay, \& Dryden, 1996; Veale, Gournay, Dryden, Boocock, \& Shah, 1996). This level of suicide attempts is among the highest of all psychiatric disorders, along with bipolar and unipolar depression (disorders in which rates of suicide attempts range from 25\%-56\%) (Jamison, 2000; Malone, Haas, Sweeney, \& Mann, 1995; Slama et al., 2004). BDD has a lifetime prevalence rate of approximately $1.7-2.4 \%$ in the population (Koran, Abujaoude, Large, \& Serpe, 2008; Rief, Buhlmann, Wilhelm, Borkenhagen, \& Brahler, 2006), and similar to hoarding disorder, is associated with poor insight (Eisen, Phillips, Cole, \& Rasmussen, 2004). In terms of age of onset, gender, and course, the average age of onset of symptoms is approximately 17 years of age (Grant et al., 2014. The gender distribution is approximately equal in males and females, and the typical course is chronic without treatment (Grant et al., 2014).

\section{Comorbidity}

Comorbidity is defined as "the presence of more than one distinct condition in an individual" (Valderas et al., 2009, p. 358). Literature differentiates between current comorbidity (more than one condition in an individual co-occurring) and lifetime comorbidity (more than one condition in the same individual over the course of their lifetime, not necessarily co-occurring at the same

\footnotetext{
${ }^{1}$ A Somatoform Disorder is defined as "the presence of physical symptoms that suggest a general medical condition and are not fully explained by a general medical condition" (American Psychiatric Association, 2000, pg. 485).
} 
time). Understanding and describing comorbidity has important implications for disorder course, prognosis, and etiology. Four bivariate models of etiological association between disorders have been described; direct causation, associated risk factors, heterogeneity, and independence (Rhee et al., 2004). In direct causation, one disorder may cause the other. In associated risk, both disorders share common risk factors. In heterogeneity, risk factors between the two disorders are not related, but can cause either disorder. In independence, symptoms of each disorder correspond to a third disease (for a review, see Valderas, Starfield, Sibbald, Salisbury, \& Roland, 2009). Research fitting quantitative models to comorbidity has found that a multivariate liability spectrum model best fits, such that there are common liability factors (associated risk factors) in multiple disorders (Krueger \& Markon, 2006). Further discussion of the intricacies of the comorbidity issue is beyond the scope of the current dissertation, but for a review, see Valderas et al. (2009).

As with most psychiatric disorders, comorbidity between the OCRDs and across psychiatric classification groups (e.g., OCRDs with depressive disorders) is quite common. Lifetime comorbidity prevalence rates from the National Comorbidity Survey Replication Study found that for those with OCD, an anxiety disorder (75.8\%) was the most common comorbid disorder, followed by mood disorders (63.3\%), impulse control disorders (55.9\%) and substance use disorders (38.6\%) (Ruscio et al., 2010). Comorbidity within the OCRDs is also quite common, with an estimated $67 \%$ of individuals with one OCRD having a lifetime history of at least one other comorbid OCRD (Lochner et al., 2014). In individuals with current OCD, approximately 18\% had current comorbid HD (Frost, Steketee, \& Tolin, 2011), 10-17\% had current comorbid GD (Odlaug \& Grant, 2008a; Woods et al., 2006), and 33\% had current comorbid BDD (Gunstad \& Phillips, 2003; Phillips et al., 2005). Comorbidity between TTM and 
ED was found in approximately $38.8 \%$ of individuals presenting with a grooming disorder (Odlaug \& Grant, 2008b).

Comorbidity among the OCRDs raises important questions regarding treatment decisions (e.g., to treat disorders sequentially versus concurrently), inclusion criteria in research studies, study recruitment, and the generalizability of research findings. It also raises important questions about the etiological association between disorders. DSM-5's decision to classify OCRDs together suggests that while distinct, conditions in this category are etiologically related. Thus, it could be expected that disorders within the OCRD category would share more general etiological factors (disorder nonspecific). However, as the lifetime prevalence rates from the National Comorbidity Survey Replication Study (Ruscio et al., 2010) highlight, OCD shares etiologic factors with other classes of disorders (e.g., anxiety and mood disorders). This will be discussed in greater detail below.

\section{First-line Treatment of the OCRDs}

The Clinical Practice Guidelines published by the American Psychiatric Association (2007) and the Canadian Clinical Practice Guidelines for the Management of Anxiety, Posttraumatic Stress, and Obsessive-Compulsive Disorders (2014) currently recognize cognitivebehavioural therapy (CBT) as a first-line treatment for OCD. Similarly, Expert Consensus Treatment Guidelines (2016) for body-focused repetitive behaviours (including TTM and ED) recommend CBT as first-line treatment. While no such guidelines currently exist for HD, examination of the results from randomized control trials reveal that CBT is also efficacious for HD (e.g., Steketee, Frost, Tolin, Rasmussen, \& Brown, 2010). CBT is considered a time-limited and goal-oriented treatment approach to therapy. CBT across the OCRDs targets the maladaptive thoughts and behaviours that are hypothesized to maintain the disorder. CBT response rates 
(measured according to clinically significant change, a conservative estimate of high end state function and reliable change) in the OCRDs range from $63 \%$ of OCD treatment completers (Abramowitz, Franklin, Schwartz, \& Furr, 2003) to 41\% of hoarding completers (Steketee et al., 2010).

In addition to psychological treatment, pharmacological treatment is also recommended by the practice guidelines cited previously (American Psychiatric Association, 2004; Katzman et al., 2014). Specifically, selective serotonin reuptake inhibitors (SSRIs) are the recommended first-line pharmacological intervention for OCD (for a review see Kellner, 2010; Richter \& Ramos, 2018). For those who fail to adequately respond to a both a first and second round of an SSRI, second-line drugs recommended include the tricyclic antidepressant, clomipramine, or the serotonin norepinephrine reuptake inhibitor (SNRI), venlafaxine (Skapinakis et al., 2016). SSRIs are also considered the first-line treatment for HD (for a review see Brakoulias, Eslick, \& Starcevic, 2015), and BDD (Ipser, Sander, \& Stein, 2009; Williams, Hadjistavropoulos, \& Sharpe, 2006). Research thus far into pharmacotherapy for the GDs has largely relied on studies with small sample sizes, and results have been largely mixed. For TTM, double blind trials revealed promising results for the tricyclic clomipramine (Swedo, Leonard, \& Rapoport, 1989), the opioid antagonist naltrexone (O’Sullivan \& Christenson, 1999), the glutamatergic agent Nacetylcysteine (Grant, Odlaug, \& Kim, 2009), and the atypical antipsychotic olanzapine (Van Ameringen, Patterson, \& Simpson, 2014). For ED, there has been some support for the SSRI fluoxetine (Bloch et al., 2001; Simeon et al., 1997). Despite the efficacy of first-line treatments for OCD, approximately $40-70 \%$ of patients fail to adequately respond to first-line interventions (Eddy, Dutra, Bradley, \& Western, 2004; Pallanti et al., 2002). Thus, a better understanding of 
the development and maintenance factors of the disorders is important to point towards novel treatment avenues and maximize existing treatment efficacy.

\section{Neurobiology of Obsessive-Compulsive and Related Disorders}

Evidence of neural systems implicated in OCRDs comes from neuroimaging, neuropsychological, and pharmacological studies. Two pathways in the fronto-striatal circuits of the brain have been most consistently implicated in OCD, including the orbito-fronto-striatal circuit (referred to as the fronto-striatal model of obsessive-compulsive disorder; FSMOCD) and the dorsolateral prefrontal-striatal circuit (DLPFC). These fronto-striatal circuits are neural pathways connecting the brain's frontal lobe regions with the basal ganglia. Structures implicated in these circuits include the dorsolateral prefrontal cortex, posterior parietal cortex, thalamus, and basal ganglia (see Menzies et al., 2008 for a review). Fronto-striatal circuits have a looped structure, with cortical input communicating with the striatum, which loops back to the cortex through the thalamus (Alexander, Crutcher, \& DeLong, 1990; Alexander, DeLong, \& Strick, 1986). These fronto-striatal circuits have been implicated in animal models of repetitive behaviour (Ridley, 1994), and their proper functioning is thought to be imperative to goaldirected behavioural regulation, such as problem solving, planning, and memory (Freyer et al., 2011; Menzies et al., 2008). Research suggests increased activity in the anterior cingulate in OCD (e.g., Del Casale et al., 2011, Machlin et al., 1991) and altered activity in the caudate (Machlin et al., 1991). Newer imaging research using functional MRI continues to support the role of the fronto-striato-thalamic model in OCD (Göttlich, Kramer, Kordon, Hohagen, \& Zurowski, 2015; Zhang et al., 2017). Additionally, meta-analyses of volumetric differences found grey matter volume deficits in the anterior cingulate cortex, orbitofrontal cortex, and parietofrontal regions, and volumetric increases in the thalamus (Radua \& Mataix-Cols, 2009; 
Rotge, Guehl, \& Diharreguy, 2009, \& Rotge et al., 2010), providing additional evidence for the implication of this circuit in OCD.

$\mathrm{HD}$ is also thought to be associated with activation in similar brain structures as OCD, with involvement of the fronto-limbic system, including the dorsolateral and ventral medial prefrontal cortex, cingulate, striatum, and medial temporal cortex (Saxena, 2008). Imaging studies in the GDs are relatively scarce; however, initial research suggests mixed support for the existence of volumetric decreases in TTM patients versus controls in frontal regions, the putamen, and cerebellum (Chamberlain et al., 2008; Keuthen, Makris, \& Schlerf, 2007; O'Sullivan et al., 1997; Stein, Forde, Anderson, \& Walker, 1997). While the neurobiology of ED is very much in its infancy, Odlaug et al. (2016), using fMRI, found functional differences in striatal circuitry and right medial frontal regions during a task of executive planning, compared to healthy controls. Structural MRI failed to find a statistically significant difference in basal ganglia volume between those with ED and healthy controls; however, the severity of skin picking symptoms was negatively correlated with cortical thickness in the left supramarginal gyrus (Harries et al., 2017). Examination of white matter in ED implicates the disorganization of white-matter tracts in the anterior cingulate cortices (Grant et al., 2013). Similar brain regions and networks appear to be implicated in BDD and OCD, with caudate and white matter volume implicated in both, although the directionality appears different. Previous findings in OCD describe a rightward shift in caudate asymmetry and reduced total white matter, while in BDD a leftward shift and increased white matter are implicated (Rauch, Shin, \& Wright, 2003; Saxena, Bota, \& Brody, 2001).

Neurochemistry involvement in the OCRDs has traditionally focused on the neurotransmitter serotonin. This interest was largely based on the finding that individuals with 
OCD responded to clomipramine preferentially over other tricyclics, indicating a selective efficacy for serotonergic medication (Zohar \& Insel, 1987). Serotonin has also been implicated in the related disorders; HD (Brakoulias et al., 2015), GD (Swedo, Rapoport, \& Leonard, 1991), and BDD (Barr, Goodman, \& Price, 1992; Marazziti, Dell'Osso, \& Presta, 1999; Phillips, Albertini, \& Rasmussen, 2002; Phillips \& Najjar, 2003). However, the serotonin hypothesis is likely an oversimplification. While SSRIs are the first line treatment in OCD, a portion of patients do not respond or only partially respond to SSRI pharmacotherapy. Thus, approximately $40-70 \%$ of patients fail to adequately respond to SSRI interventions alone (Pallanti et al., 2002), indicating that other neurotransmitters systems, such as dopamine and glutamate, may also be implicated (Bloch et al., 2006; Dold, Aigner, Lanzenberger, \& Kasper, 2013; Goodman, McDougle, \& Price, 1990; Grados, Atkins, Kovacikova, \& McVicar, 2015; McDougle, Goodman, \& Leckman, 1994; Pittenger, 2015; Pittenger, Bloch, \& Williams, 2012; Stein \& Hollander, 1992; Van Ameringen, Mancini, \& Oakman, 1999; Van Ameringen, Mancini, \& Patterson, 2006; Veale et al., 2014), particularly for the GDs (Grant et al., 2009; Grant, Odlaug, Chamberlain, \& Kim, 2010; Stein \& Hollander, 1992; Van Ameringen et al., 1999; 2006).

\section{Genetic Factors}

OCD has reliably demonstrated moderate heritability, with twin studies demonstrating concordance rates ranging from $80-87 \%$ in monozygotic twins and $47-50 \%$ in dizygotic twins (Cary \& Gottesman, 1981; Inouye, 1965). Familial studies also point to a genetic component in OCD, with OCD being 5 to 7 times more common in first-degree relatives of individuals with OCD than in first-degree relatives of controls (Nestadt et al., 2000; Pauls, Alsobrook, Goodman, Rasmussen, \& Leckman, 1995). More contemporary behavioral-genetic studies suggest that 26$47 \%$ and $27-65 \%$ of variance is accounted for by genetics, in adults and children/adolescents, 
respectively (Eley, Lichtenstein, \& Moffitt, 2003; Hudziak et al., 2004; Hur \& Jeong, 2008;

Jonnal, Gardner, Prescott, \& Kendler, 2000; van Grootheest, Bartels et al., 2007; van Grootheest, Cath, Beekman, \& Boomsma, 2007; van Grootheest et al., 2008). However, despite considerable evidence pointing to the heritability of this disorder, the search for genes has been fraught with inconsistent findings resulting in minimal replication success for both chromosomal and candidate gene findings (Pauls, 2010; Sklar, 2002).

Unlike simple Mendelian genetics, where one gene produces one phenotype, psychiatric disorders are complex genetic disorders, where multiple genes are responsible for phenotypic expression. Thus, each gene confers only a small risk and is part of a biopsychosocial setting that includes both genetic and environmental factors that influence phenotypic expression. As each gene likely confers only small to moderate risk, the aforementioned replication difficulties are likely due, in part, to studies lacking sufficient power and the complex etiologic relationship with environmental factors. The first comprehensive meta-analysis of candidate gene studies sought to improve aforementioned issues related to power, and revealed that polymorphisms in serotonin modulation and catecholamine regulation (in males) were related to OCD, with trends for two dopamine polymorphisms and one glutamate related polymorphism (Taylor, 2013).

Two genome-wide association studies (GWAS) have been completed in OCD (Mattheisen et al., 2015; Stewart et al., 2012). The first GWAS revealed one single nucleotide polymorphism (SNP) achieved genome-wise significance in the trio analysis. This SNP is thought to be related to the BTBD3 gene, a transcription factor. However, this SNP lost genomewise significant when combined with case-control samples. Case-control analysis revealed two SNPs associated with the DLGAPI gene, important in glutamate signaling (Stewart et al., 2012). The second GWAS failed to find genome-wide significance but noted a signal on chromosome 9 
near $P T P R D$, thought to be implicated in the differentiation of glutamatergic synapses, and found trending significance levels for genes associated with DLGAPl (Mattheisen et al., 2015). As both GWAS studies produced slightly differing results, a meta-analysis of both GWAS studies was conducted (Arnold et al., 2017). While no SNPs reached statistical significance, strong signals were found for several genes implicated in previous GWAS, including DLGAP1 and PTPRD, with GRIK2, FAIM2 and CDH2O.

Contemporary twin research has sought to examine the relative contribution of genetic and environmental etiological factors in OCD. Sources of variation in complex traits include additive genetic effects (genes are independent and additively combine), nonadditive genetic effects (interactions between genes), shared environment (experiences shared by both twins), and nonshared environment (experiences specific to one twin). Evidence suggests a primarily additive model in OCD, with additive genetic effects accounting for $29 \%$ of variance (Bolton, Rijsdijk, O’Connor, Perrin, \& Eley, 2009; Tambs et al., 2009). This finding was confirmed by meta-analyses of twin (Taylor, 2011) and molecular genetic association studies (Taylor, 2013). Examination of shared and nonshared environmental factors found that $51 \%$ of the variance in OCD symptoms was accounted for by the nonshared environmental factors, while the shared environment accounted for $0 \%$ of the variance (Taylor, Jang, \& Asmundson, 2010). The finding that shared environment accounted for little of the variance (0-7\%) has occurred in multiple twin studies (Eley et al., 2003; Hudziak et al., 2004; Hur \& Jeong, 2008; Jonnal et al., 2000; van Grootheest, Bartels et al., 2007; van Grootheest, Cath et al., 2007; van Grootheest et al., 2008), and suggests that the environmental contribution is largely nonshared.

Twin studies have identified that in the etiology of psychiatric disorders, both nonspecific factors (etiological factors of many disorders) and disorder-specific factors (etiological factors 
specific to the diagnosis) are implicated (Kendler \& Prescott, 2000). Indeed, results suggest that OCD symptoms arise from both general and specific genetic and environmental factors (Taylor et al., 2010). Examples of general environmental factors include perinatal complications, traumatic life events, and streptococcal infection (Grisham et al., 2011; Moffitt, Caspi, \& Rutter, 2005; Murphy, Timpano, Wheaton, Greenberg, \& Miguel, 2010). Examples of specific environmental factors include appearance-related bullying in BDD (Buhlmann, Cook, Fama, \& Wilhelm, 2007; Phillips, 2009) and trauma-related loss in HD (Landau, Lervolino, \& Pertusa, 2011). Preliminary research suggests that environmental influences are largely disorder specific (Monzani et al., 2014).

While it is believed that the heritability of the related disorders is similar, and perhaps slightly higher in hoarding disorder (Mathews et al., 2007), the search for genes in the related disorders is very much in its infancy. The inclusion of HD, GDs, and BDD within the same category in DSM-5, OCRDs, suggests that while these are now considered distinct conditions, the disorders are suspected to be etiologically related. Thus, it could be expected that disorders in the OCRD category would share general etiological factors; however, as aforementioned OCD shares features with other classes of disorders as well (e.g., mood, anxiety), which raises the question about the extent to which general and specific etiological factors contribute to a specific disorder or class of disorders. This has led some to ponder etiological-based disorder classification as an alternative to the current symptom-based classification currently utilized (e.g., Taylor et al., 2010).

In the first multivariate twin study to include all the OCRDs in the same analysis, results indicated moderate heritability for all OCRDs: OCD (48\%), HD (51\%), TTM (32\%), ED (47\%), and BDD (43\%). Similar to the aforementioned OCD findings, in the related disorders, 
nonshared environmental factors accounted for the remaining variance, with an insignificant contribution from the shared environment (Monzani et al., 2014). This multivariate twin study also allowed for examination of the etiological architecture of the OCRDs. Results revealed two common liability factors. The first factor had a substantial genetic loading (63\%) and loaded on to all of the OCRDs; the second factor also had a substantial genetic loading (74\%) but was specific to TTM and ED (Monzani et al., 2014). This finding suggests that the first factor is a general etiological factor that confers risk to all OCRDs, while the second factor is specific to TTM and ED.

\section{Endophenotypes}

One approach in the search for candidate genes is to focus on vulnerability traits (Leboyer et al., 1998). Endophenotypes are defined as intermediary phenotypes that connect "the pathway between disease (phenotype) and distal genotype” (Gottesman \& Gould, 2003, p. 636). It is hypothesized that the genetic contributors of endophenotypes may, in fact, be less complex than the illness phenotype (Gottesman \& Gould, 2003). To be considered an endophenotype, a trait must exhibit several properties (Cannon \& Keller, 2006): 1) Endophenotypes should be heritable; 2) Endophenotypes should be associated with the etiology of the disorder rather than being a symptom of a disorder; 3) A complex disorder is composed of numerous endophenotypes; 4) Endophenotypes should vary continuously in the general population; 5) The measurement of endophenotypes should be considered across several levels of analysis; and 6)

Genetically related disorders should have some endophenotypic overlap. Other similar definitions have been posited by Gottesman and Gould (2003) and Preston and Winberger (2005). 
More recent research, however, noted that the aforementioned definitions of endophenotype "do not discriminate between liability-index and a mediational model for endophenotypes" (Kendler \& Neale, 2010, p.789). The traditional conceptualization of an endophenotype as conceptualized by Gottesman and Shields (1972) posited that an endophenotype is a risk indicator, in which shared genetic variance increases risk for both psychopathology and endophenotype. In an expanded conceptualization of endophenotype, the mediational model posits that endophenotypes are intermediary between environmental contributors and psychopathology, such that genetic variance causes variation in the endophenotype, which causes variation in psychopathology (Kendler \& Neale, 2010). This distinction has important implications for the proposed etiology of psychopathology and predicts different treatment targets. For example, the mediating variable model suggests that treatment could target the endophenotype, such that reducing levels of the endophenotype would lower the risk of psychopathology (Kendler \& Neale, 2010). The risk indicator model, by contrast, suggests that the treatment target would be to correct the genetic liability (Kendler \& Neale, 2010).

Contemporary criteria for candidate endophenotypes (CE) set out by Taylor (2012, p. 259), including conceptualizations from Cannon and Keller, 2006; Gottesman and Gould, 2003; and Kendler and Neale, 2010 include:

The CE is associated with causes of the disorder; 2)The CE is trait-like; it is stable within individuals and varies quantitatively in the general population; 3) If the disorder is heritable, then so is the $\mathrm{CE}$, and they have a shared genetic basis; 4) If the disorder is heritable, then the $\mathrm{CE}$ and disorder should co-segregate and, for probands diagnosed with the disorder, their first degree relatives should have a higher risk of 
developing the disorder compared to the general population; 5) If the disorder is influenced by environmental factors, then so is the CE, and they should have a common environmental etiology.

While these contemporary criteria are similar to the aforementioned historical conceptualization of an endophenotype (e.g., Gottesman and Shields, 1972), they importantly allow for a mediational model and do not imply that endophenotypes are necessarily etiologically related to a disorder. With the utilization of more flexible criteria, the CE may be a marker of a causal mechanism without itself having causal influence.

\section{Candidate endophenotypes for OCD}

The search for CEs in OCD is intimately linked to underlying etiological models of disorder development. As previously reviewed, aberrant frontal-thalamo-striatal circuitry has long been implicated in OCD. Arising from this, the search for CEs has included markers of dysregulation of this circuit, including neurocognitive markers (e.g., deficits in executive functioning), neuroimaging markers, and neurotransmitter markers (e.g., Aouizerate et al., 2004; Cavedini, Gorini, \& Bellodi, 2006; Menzies et al., 2008). The cognitive theoretical perspective for the etiology of OCD posits that learning experiences influence dysfunctional beliefs, which lead to OCD in the presence of an environmental stressor (e.g., Clark, 2004; Frost \& Steketee, 2002; Rachman, 1997). This approach has also generated potentially important CEs.

While the two aforementioned theoretical perspectives have robust empirical support, there are other well-supported etiological perspectives (e.g., sociocultural factors; Dhuri \& Parkar, 2014; Frost, Hahart, \& Rosenblate, 1994). Indeed, recent behavioural-genetic work suggests that current models fail to capture the nuanced architecture informed by twin studies, suggesting the need for a more comprehensive biopsychosocial approach (Taylor, 2011). While 
it would be ideal to explore neurocognitive and dysfunctional belief CEs in conjunction with other putative CEs in a truly biopsychosocial approach, a study of this magnitude is beyond the scope and practical limitations of the current investigation, and likely necessitates the collaboration across sites. As such, the current dissertation focused specifically on neurocognitive and dysfunctional beliefs, as these have been suggested to be amongst the most robust endophenotypes, they are more readily translatable to clinical use (e.g., do not require sophisticated technology and are less costly), and have not been explored broadly across the OCRDs. These two bodies of literature are reviewed below. For a review of neurotransmitter, EEG, and neuroimaging candidate endophenotypes in OCD, see Taylor (2012). This dissertation will contribute to knowledge by taking a dimensional approach, looking at similarities/differences across the OCRDs to better understand the relationships and shared/specific underlying factors.

\section{Neuropsychological Candidate Endophenotypes.}

It is hypothesized that neurocognitive tasks are good endophenotypes, as the genes implicated in psychiatric disorders likely impact neural systems that mediate neurocognitive functioning (Cannon, Gasperoni, van Erp, \& Rosso, 2001). As reviewed above, the pathway of the brain most consistently implicated in these disorders is the orbito-fronto-striatal circuit, which is further supported by cognitive impairments in these domains (Aouizerate et al., 2004; Cavedini et al., 2006; Menzies et al., 2008).

Despite substantial work examining neurocognition in OCD, this field of study has been fraught with inconsistent findings. Indeed, a review of neuropsychological performance in over 50 OCD studies concluded that "results of previous studies do not present a clear and specific neuropsychological profile" (Kuelz, Hohagen, \& Vonderholzer, 2004, p. 230). A review and 
meta-analysis of the current state of the literature has highlighted that neuropsychological factors, clinical factors, and general factors are negatively influencing the replicability of findings (Abramovitch et al. 2015; Kuelz et al., 2004), as confounding variables and methodological limitations make it difficult to compare across studies (Abramovitch et al., 2015). General factors include a failure to correct for multiple comparisons in $82 \%$ of studies reviewed (Abramovitch et al., 2015). This is particularly problematic in neuropsychology research where tasks have numerous dependent variables, effect sizes tend to be small, and power is low. Recruitment was cited as another important factor limiting replicability and generalizability, via selection bias of those recruited from speciality clinics versus the community. Often those recruited from a speciality clinic are treatment seeking, but also present with more severe symptomatology and a more complex clinical picture (McConaughy \& Achenbach, 1994).

Clinical factors that have contributed to the replicability problem include not reporting symptom severity or not examining how severity is linked to performance, failure to control for depressive symptoms, the heterogeneous nature of OCD, the historical inclusion of hoarding as a symptom of OCD, and varying medication status of sample participants. Neuropsychological factors that have been cited to influence the mixed findings in the field include differing education levels of sample participants, a lack of validation of tests in non-English speaking countries, and the use of differing tests to assess a single construct (Abramovitch et al. 2015; Kuelz et al., 2004).

Thus, an attempt to summarize the previous literature on proposed cognitive endophenotypes of the OCRDs is provided below, but the review is fraught with inconsistent 
findings. Where possible, results of review studies and meta-analytic data are included to provide summaries of discrepant findings.

\section{Inhibitory dysfunction.}

Inhibitory dysfunction has been posited to be a particularly promising endophenotype of OCD. Given the illness phenotypes of the OCRDs, which include difficulties suppressing inappropriate repetitive behaviours (e.g., hair pulling, skin picking, and compulsive behaviours), it has been suggested that there may be an underlying dysregulation in inhibitory control processes (Chamberlain, Blackwell, Fineberg, Robbins, \& Sahakain, 2005). Additionally, neuroimaging studies indicate that inhibitory dysfunction (e.g., selecting information from competing responses; selective attention) is associated with similar brain regions as OCD, including activation of the inferior frontal gyrus, anterior insula, anterior cingulate cortex, dorsolateral prefrontal cortex, and parietal regions (Nee, Wager, \& Jonides, 2007; Sebastian et al., 2013; Wager et al., 2005).

\section{Response inhibition.}

Response inhibition, a measure of executive control, is defined as the ability to suppress a behaviour that is no longer adaptive, appropriate, or required in accordance with changing situational demands (Aron, Fletcher, Bullmore, Sahakian, \& Robbins, 2003; Chambers, Garavan, \& Bellgrove, 2009; Logan, Cowan, \& Davis, 1984). Response inhibition is not a singular construct but rather consists of interference control, action restraint, and action cancellation. Interference control is defined as the cognitive ability to prevent interference due to competing stimuli (e.g., focusing on one stimuli while ignoring others, such as focusing on colour while ignoring number; Nigg, 2000). Action restraint is defined as "the ability to withhold a strong response tendency" while action cancellation is defined as "the ability to cancel an ongoing 
action" (Schachar et al., 2007, p. 229). Response inhibition is most commonly measured utilizing either the Stop Signal Task, which measures one's ability to inhibit an ongoing speeded motor response (Lipszyc \& Shachar, 2010), or the Go/No Go Task, in which individuals make a motor response as quickly as possible in response to a target cue, but withhold that response when a non-target cue is presented. The Go/No Go Task has been hypothesized to measure action restraint, while the Stop Signal Task is hypothesized to measure action cancellation (Schachar et al., 2007).

Additional evidence of response inhibition as a promising endophenotype comes from the finding that unaffected first-degree relatives of individuals with OCD displayed a similar impairment in response inhibition (Chamberlain, Fineberg, Blackwell, Robbins, \& Sahakian, 2006; Menzies et al., 2007). Furthermore, in both those with OCD and their unaffected firstdegree relatives, this deficit in response inhibition is associated with reduced volume of grey matter in the orbitofrontal region (Menzies et al., 2007).

A synthesis of the literature suggests that individuals with OCD display impaired performance on the Stop Signal Task (Chamberlain, Fineberg, Menzies et al., 2007; Johannes et al., 2001; Krikorian, Zimmerman, \& Fleck, 2004; Penades et al., 2007; Woolley et al., 2008); however, not all studies have found such an association (Boone, Ananth, Philpott, Kaur, \& Djenderedjian, 1991; Kalanthroff et al., 2016; Krishna et al., 2011; Rao, Reddy, Kumar, Kandavel, \& Chandrashekar, 2008). Nevertheless, a recent meta-analysis examining response inhibition across a variety of psychopathologies reported that individuals with OCD display a deficit in action cancellation (as measured by the Stop Signal Task) with a medium effect size (Hedge's g = 0.77) (Lipszyc \& Schachar, 2010) and a deficit in action restraint (as measured by the Go/No Go Task) with a small effect size (Hedge's $g=0.37$ ) compared to nonpsychiatric 
controls (Wright, Lipszyc, Dupuis, Thayaparajah, \& Schachar, 2014). Taking the evidence together, the authors suggest that action cancellation, as opposed to action restraint deficits, may be the most relevant to OCD (Wright et al., 2014).

With respect to the other OCRDS, the majority of studies find impaired response inhibition in ED and TTM (Grant et al., 2011; Odlaug, Chamberlain, \& Grant, 2010; Odlaug, Chamberlain, Schreiber, \& Grant, 2013), with some indications that individuals with TTM may be more impaired than individuals with OCD in action cancellation (Chamberlain et al., 2006). However, not all studies have found a deficit among the body-focused disorders (Grant, Odlaug, \& Chamberlain, 2011; Snorrason, Smari, \& Olafsson, 2011).

Preliminary results examining the association between response inhibition and HD are mixed, with some studies finding that those with HD perform worse on measures of response inhibition (Morein-Zamir et al., 2014; Rasmussen, 2012) compared to nonpsychiatric controls, while other studies have found no such association (Blom et al., 2011; Grisham, Norberg, Williams, Certoma, \& Kadib, 2010; Tolin, Witt, \& Stevens, 2014). To date, research on response inhibition in BDD is scarce; however, preliminary evidence indicates impairment in response inhibition compared to controls (Jefferies-Sewell, Chamberlain, Fineberg, \& Laws, 2017).

\section{Interference control.}

Interference control is defined as "suppressing a stimulus that pulls for a competing response so as to carry out a primary response" (Nigg, 2000, p. 222). This construct is most often measured using the Stroop Colour and Word Test (Golden, 1976). During this task, on the interference trial, participants read aloud colour names printed in another ink colour. While this task measures interference control, it also taps selective attention and cognitive flexibility. The bulk of published literature on the Stroop suggest that those with OCD demonstrate worse 
performance compared to non-psychiatric controls, marked by increased reaction times during incongruent trials (Bannon, Gonsalvez, Croft, \& Boyce, 2002; Penades et al., 2007; Nabeyama et al., 2008; Nakao et al., 2009; Schlosser et al., 2010).

Research on interference control in the related disorders is very much in its infancy. Preliminary research suggests deficits in interference control in individuals with TTM (Bohne et al., 2005; Stanley, Hannay, \& Breckenridge, 1997). Findings from studies on interference control in HD patients have been mixed, with some studies finding those with HD have worse performance on the Stroop (de Kort, 2012; Mackin, Arean. Delucchi, \& Mathews, 2011), while others have found no such performance deficit (Sumner, Noack, Filoteo, Maddox, \& Saxena, 2016; Tolin, Meunier, Frost, \& Steketee, 2011). Research examining interference control in BDD has not found difference on interference trials in those with BDD compared to nonpsychiatric controls (Bailey, 2002).

\section{Cognitive flexibility.}

\section{Set-Shifting.}

Set-shifting is a measure of cognitive flexibility that is thought to correspond to an individual's ability to shift attention when environmental incentives change. Given that individuals with OCRDs often perseverate in their thoughts and behaviour, set-shifting has been hypothesized as a potentially important endophenotype. Although set-shifting is commonly associated with the frontal regions of the brain, attributing difficulties to a particular brain region is overly simplistic, as the ability to switch attention most likely involves the interaction of multiple brain regions (Stuss \& Benson, 1986).

The Wisconsin Card Sorting Task (WCST; Heaton, Chelune, Talley, Kay, \& Curtiss, 1993) is one of the most widely used measures of set-shifting, in which participants must identify 
a rule and change their response when the rule changes. Thus, it is believed that the WCST assesses the inability to inhibit a previously correct response, leading to behavioural perseveration (Sanz, Molina, \& Calcedo, 2001). Other measures often used to study set-shifting include the Object Alternation Task (OAT; Freedman, 1990), in which participants have to learn a contingency to find a hidden coin under one of two cups, and the Intra-dimensional/Extradimensional Set Shift Task (ID/ED; Cambridge Neuropsychological Test Automated Battery CANTAB, 2006), which is a computerized analogue of the WCST in which participants must learn which stimulus shape is correct based on shifting rules.

While some studies have reported impaired set-shifting in OCD (Boone et al., 1991; Hymas, Lees, Bolton, Epps, \& Head, 1991; Lucey et al., 1997; Okasha et al., 2000), a comparable number of negative findings have emerged (Abbruzzese, Bellodi, Ferri, \& Scarone, 1995; Abbruzzese, Ferri, \& Scarone, 1995, 1997; Deckersbach, Otto, Savage, Baer, \& Jenike, 2000; Gross-Isseroff et al., 1996; Laniti, 2005; Moritz et al., 2001, 2002; Zielinski, Taylor, \& Juzwin, 1991). A meta-analytic review of WCST performance in OCD, which examined three WCST outcome variables (categories completed, perseverative errors, total errors), found small to moderate effect sizes indicating impaired performance in those with OCD, compared to controls (mean effects : $0.23,0.25$, and 0.32 respectively). However, when overall psychomotor speed was included, the WCST was not disproportionately impaired, potentially indicating a more generalized cognitive impairment (Henry, 2006). A subsequent meta-analysis that examined set-shifting across measures found a medium effect size, indicating that those with OCD are impaired on measures of set-shifting compared to non-psychiatric controls (Abramovitch, Abramowitz, \& Mittelman, 2013). 
Examination of set-shifting in the OCRDs revealed similar discrepancies in HD, with most (Ayers et al., 2013; McMillan, 2013; Morein-Zamir et al., 2014), but not all (Lawrence et al., 2006; Tolin, Villavicencio, Umback, \& Kurtz, 2011) studies finding those with HD are impaired on the WCST. Indeed, failure to maintain set (failure to continue with a successful rule prior to the rule change) significantly predicts hoarding symptom severity (McMillan, Rees, \& Destell, 2013). However, when examined using the IE-ED, no differences between hoarding patients and controls have emerged (de Kort, 2012; Grisham, Norberg, Williams, Certoma, \& Kadib, 2010).

The majority of studies have found no set-shifting impairment in ED and TTM patients, using either the WCST or the ID/ED (Bohne et al., 2005; Chamberlain et al., 2006; Grant et al., 2011; Odlaug et al., 2010); however, Bohne et al. (2005) found increased perseveration in TTM patients on the Object Alternation Task compared to non-psychiatric controls, and an additional study found impaired set-shifting on the ID/ED compared to nonpsychiatric controls (Odlaug et al., 2013). The one study to date to examine set shifting in BDD found no significant difference between the set shifting performance of BDD patients and nonpsychiatric controls (Laniti, 2005).

\section{Memory.}

Based on the phenomenology of OCD and HD, memory impairment has been another hypothesized endophenotype of importance. In particular, for a subset of individuals with OCD, repeated checking is often seen as a compulsion. Checking compulsions are often reported by patients to be due to issues with their memory and/or lack of confidence in their memory (e.g., repeatedly checking to see if the stove was turned off; Sher, Frost, \& Otto, 1983). Decreased levels of memory confidence in OCD has been reliably reported (Hermans, Engelen, Grouwels, et al., 2008; MacDonald, Antony, MacLeod, \& Richter, 1997; Moritz \& Jaeger, 2018). 
Interestingly, repeated checking is associated with less vivid memories of the checking event (van den Hout \& Kindt, 2003). However, OCD is a heterogeneous disorder, and how memory may be implicated in the other symptom clusters remains an important research question.

Similarly, memory has been implicated in HD. According to the cognitive-behavioural theory of HD, memory plays an important role in the disorder. Specifically, it has been hypothesized that individuals with hoarding disorder have cognitions about memory that contribute to the development and maintenance of symptoms, including lack of confidence in their ability to remember important information and overestimating the importance of remembering details (Frost \& Hartl, 1996). In support of this hypothesis, both research findings and clinical anecdotes suggest that those with HD report significantly less confidence in their memory and more catastrophic interpretations regarding forgetting (e.g., 'I won't remember an important event or person'), resulting in a strong desire to keep possessions in sight, and greater use of memory aids compared to nonpsychiatric controls (Hartl, Duffany, Allen, Steketee, \& Frost, 2005; Hartl et al., 2004). However, whether there is a true memory impairment, or whether these phenomena are a function of memory confidence is an important area of future research. Neurocognitive findings related to memory are reviewed below.

Nonverbal memory.

Nonverbal memory is memory for information and events that are not language encoded (e.g., visual memory). Nonverbal memory has most commonly been assessed in the OCRDs using the Rey Osterrieth Complex Figure Test (RCFT; Osterrieth, 1944) or the Benton Visual Retention Test (BVRT; Benton, 1974). During the RCFT, a complex line drawing is presented. The participant first copies the image, and then is asked to reproduce the image from memory 
immediately after copying and after a delay. During the BVRT, 10 designs are presented to the participant, and they are asked to reproduce them from memory.

The relationship between OCD and nonverbal memory is complex, and results from individual studies have been mixed, with some studies finding deficits in OCD patients compared to nonpsychiatric controls (Deckersbach et al., 2000; Lacerda et al., 2003; Savage et al., 1999), while other studies report intact performance (Bédard, Joyal, Godbout, \& Chantal, 2009; Simpson et al., 2006) across a variety of nonverbal memory tasks, including the RCFT, the Tactual Performance Test, and the Benton Visual Retention Test. Part of the discrepancy in findings may be due to the fact that nonverbal memory is not a unitary construct, and successful completion of the tasks utilized to measure nonverbal memory (e.g., RCFT) requires multiple processes. A review of nonverbal memory in OCD revealed that individuals with OCD performed significantly worse than healthy controls, making more errors and using poor organizational strategies (Kuelz et al., 2004). There is some indication that memory dysfunction may result from the inability of OCD participants to utilize organizational strategies that improve recall (Deckersbach et al., 2000; Savage et al., 1999, 2000). Thus, it has been hypothesized that memory may be intact in $\mathrm{OCD}$, but deficits in the ability to use organizational strategies may result in encoding and information recall difficulties. However, these results have not consistently been replicated (e.g., Simpson et al., 2006). Nevertheless, a recent meta-analysis revealed a large effect size when examining non-verbal memory impairment in OCD compared to controls (Cohen's $d=.76$; Abramovitch et al., 2013).

The Spatial Working Memory Task (SWM) from CANTAB (Cambridge Cognition Ltd, 2006) examines retention and manipulation of visuospatial information. Specifically, participants are asked to search coloured boxes on a computer screen to see if a token is hidden behind the 
boxes. The token is never hidden in the same place twice, thus requiring participants to remember where the token has previously been placed. Results on this task for individuals with OCD have also been mixed, with some studies finding that participants with OCD make more errors (Chamberlain et al., 2007; Dittrich et al., 2010; Dittrich, Johansen, Landro, \& Fineberg, 2011; Nedeljkovic et al., 2009; Purcell, Maruff, Kryios, \& Pantelis, 1998a, b) while other studies have found no significant difference between those with OCD and controls (Barnett et al., 1999; Nielen \& den Boer, 2003; Simpson et al., 2006). Based on the theoretical importance of memory for individuals with OCD and primary checking symptoms, Nedelikovic et al. (2009) examined SWM performance as it related to OCD symptom subtype. They found inflated error rates in checkers and those with mixed compulsions. A subsequent study investigating nonverbal memory in OCD subtypes found impairment in spatial recognition memory whereas spatial working memory (performance accuracy) was intact. Subtype analyses revealed recognition accuracy and recognition time were both impaired only in those with contamination symptoms (Dittrich et al., 2011).

Neurocognitive studies find some support for a nonverbal memory impairment in HD, although the picture is not clear, with some studies finding poorer recall in HD compared to controls (Hartl et al., 2004; Testa, Pantellis, \& Fontanelle, 2011), while others have not (Sumner et al., 2016; Tolin et al., 2011). Thus, it is possible that, similar to the findings in OCD, mixed findings could be a result of poorer organizational strategy (Tolin et al., 2011) and not a memory deficit, per se.

Very few studies have examined nonverbal memory in the other OCRDs. Preliminary results in TTM are mixed, with some studies (but not all; e.g., Bohne et al., 2005) finding impairment in nonverbal memory in TTM patients compared to nonpsychiatric controls 
(Chamberlain, Fineberg, Blackwell et al., 2007; Keuthen, Savage, O’Sullivan, Brown, \& Shera, 1996). Preliminary research suggests impairment in nonverbal memory for those with BDD, with results demonstrating significantly poorer recall than controls (Deckersbach et al., 2000; Dunai, Labuschagne, Castle, Kyrios, \& Rossell, 2010) and more errors on the SWM task, particularly with increased task difficulty (Dunai, et al., 2010). The one study examining memory in ED found that those with excoriation showed deficits in non-verbal memory (Nielsen. Fruensgaard, $\&$ Hjortsho, 1980); however, due to the paucity of studies, this remains an area for future research.

\section{Verbal memory.}

Verbal memory refers to memory of words, or that which is encoded by language. The California Verbal Learning Test (CVLT; Delis, Kramer, Kaplan, \& Ober, 1987) is one of the most common measures of verbal memory. Participants are read a list of 16 words from four categories. This list is read to participants on five sequential trials, with the participant recalling as many items as possible after each administration. Again, results on this and similar tasks have been mixed, with some studies finding impairment in verbal memory in OCD (Cabrera, McNally, \& Savage, 2001; Cohen et al., 1996; Deckersbach et al., 2000; Savage et al., 2000), while others have not (Bédard et al., 2009; Bohne et al., 2005; Boone et al., 1991; Burdick, Robinson, Malhotra, \& Szeszko, 2008; Christensen, Kim, Dysken, \& Hoover, 1992; deGeus, Denys, Sitskoom, \& Westenberg, 2007; Martin, Wiggs, Altemus, Rubenstein, \& Murphy, 1995; Mataix-Cols et al.,1999 Zielinski et al., 1991). A recent meta-analysis found only a small effect size for decreased verbal memory in OCD patients compared to controls, Cohen's $\mathrm{d}=.33$ (Abramovitch et al., 2013). 
One hypothesis for the mixed findings is that results may depend on whether or not the task benefits from semantic clustering (e.g., grouping words of similar categories to assist with retention and recall). Providing preliminary support for this hypothesis, Cabrera et al. (2001) found that individuals with OCD showed a selective deficit for integration of semantic units in a sentence, but their memory for the sentences did not differ from healthy controls. However, this is not supported by negative findings on the CVLT, a task that includes semantic clustering (e.g., Bedard et al., 2009; Burdick et al., 2008; de Geus et al., 2007). Alternatively, knowing that organizational aspects of memory are closely linked to executive functioning (Savage, 1997), it has been hypothesized that deficits on memory tasks may actually reflect executive dysfunction.

Examination of verbal memory in the related disorders revealed mixed findings of a verbal memory deficit for HD patients compared to controls, with one study indicating verbal memory impairment (Hartl et al., 2004), while subsequent studies found no significant association (Fitch, 2011; Sumner et al., 2016; Tolin et al., 2011). Preliminary evidence suggests that those with TTM do not exhibit verbal memory impairments (Bohne et al., 2005; Keuthen et al., 1996), but there is some indication of impairment in verbal memory for those with BDD (Deckersbach et al., 2000; Dunai et al., 2010). The one study to examine verbal memory in ED found impairment in verbal learning relative to controls (Nielsen et al., 1980).

\section{Decision-Making.}

Decision-making is another proposed endophenotype that has a hypothesized importance in OCD (Cavedini et al., 2002; Sachdev \& Malhi, 2005). This is based on both self-report indecisiveness as a reported clinical symptom, and the fact that decision-making may also rely on executive functioning and the ability to evaluate and manipulate environmental information, which is thought to be impaired in OCD (Dittrich \& Johansen, 2013). Decision-making also has 
hypothesized importance for HD. Difficulty deciding what to discard is a hallmark of the disorder (Frost \& Hartl, 1996). Additionally, decision-making is explicitly targeted in the goldstandard cognitive-behavioural interventions for HD (e.g., Steketee \& Frost, 2003; Steketee et al., 2010).

Despite the phenomenological underpinnings, results thus far from studies on decisionmaking paradigms in OCD have been decidedly mixed, with some findings indicating that individuals with OCD are significantly impaired (Cavedini et al., 2002; Kocak, Nalcaci, Ozguven, Nalcaci, \& Ergene, 2010; Viswanath, Reddy, Kumar, Kandavel, \& Chandrashekar, 2009), while an equal number of others report no statistically significant difference between individuals with OCD and healthy controls on measures of decision-making (Lawrence et al., 2006; Nielen, Veltman, De Jong, Mulder, \& den Boer, 2002; Watkins et al., 2005). Mixed findings also emerge when examining unaffected first degree relatives of individuals with OCD. To illustrate, Cavedini, Zorzi, Piccinni, Cavallini, \& Bellodi, (2010) found that first-degree relatives demonstrated statistically significant impairment in decision-making compared to controls, while Chamberlain et al. (2006) found no such impairment. Nevertheless, Sachdev and Malhi (2005) found that OCD patients were significantly more impaired on decision-making tasks compared to patients with schizophrenia, or panic disorder. Such findings led Chamberlain et al. (2005) to identify decision-making as an important area of future research in OCD, with the recommendation that a variety of decision-making measures be used.

Part of the discrepancy in decision-making findings may relate to conceptualizing decision-making as a unitary construct when, in fact, decision-making is likely comprised of many different facets, including organizational strategies, impulsivity, risk perception, and ambiguity (Dittrich \& Johansen, 2013; Olley, Malhi, \& Sachdev, 2007; Starcke, Tuschen- 
Caffier, Markowitsch, \& Brand, 2010; Stern et al., 2013). In addition, the differing measures utilized to measure decision-making, and the measures' lack of ecological validity, could also be responsible for replication difficulties. Gambling tasks are the most commonly utilized measures of objective decision-making; however, it is possible that decision-making tasks primarily involving risk taking may not accurately reflect how individuals make decisions in daily life.

Despite the hypothesized importance of decision-making in HD based on the phenomenology of the disorder (e.g., difficulty making decisions about possessions to discard), evidence of decision-making deficits using the gambling tasks has been unconvincing, with the majority of studies finding no difference between those with HD and healthy controls (Blom et al., 2011; Grisham, Brown, Savage, Steketee, \& Barlow, 2007; Grisham et al., 2010; Tolin \& Villavicencio, 2011). This has led some to hypothesize that decision-making deficits in HD may only appear when making personally relevant decisions (Grisham et al., 2010). However, when examining decision-making using the latency to make a decision (e.g., how long a decision takes), results suggest that those with HD take significantly longer to make decisions (Tolin, Kiehl, Worhunsky, Book, \& Maltby, 2009; Tolin et al., 2012). Additionally, those with HD consistently self-report indecisiveness on the Indecisiveness Scale (Frost \& Shows, 1993) relative to controls across the four reported studies (Grisham et al., 2010; Steketee et al., 2003; Tolin \& Villavicencio, 2011; Wincze, Steketee, \& Frost, 2007). A recent review of cognitive performance in HD highlighted that although results thus far on decision-making paradigms have not supported indecisiveness as a core feature of HD, extant studies do not specifically address the indecision about possessions one might hoard that was reported by Frost and Hartl (1996) (Woody, Kellman-McFarlane, \& Welsted, 2014). Thus, it is possible that decision-making deficits may be exclusive to decisions regarding personal possessions. Therefore, it is possible 
that the extant studies using gambling paradigms have not directly measured this aspect of decision-making.

Decision-making in the other related disorders has not been well studied. One study suggested no decision-making impairment in TTM patients relative to controls (Chamberlain et al., 2007). To the author's knowledge, decision-making has not been studied in ED or BDD and remains an area for future research.

\section{Dysfunctional Beliefs as Candidate Endophenotypes}

As aforementioned, the second theoretical perspective for the etiology of OCD posits that learning experiences influence dysfunctional beliefs, which lead to OCD in the presence of an environmental stressor (e.g., Clark, 2004; Frost \& Steketee, 2002; Rachman, 1997). In other words, learning models of the OCRDs conceptualize dysfunctional beliefs as responsible for the development and maintenance of the disorders under certain environmental situations.

Contemporary research suggests that dysfunctional beliefs are promising CEs for OCD, as they fit the endophenotype criteria aforementioned (e.g., Taylor, Abramowitz, McKay, \& Cutier, 2012). Specifically, research examining dysfunctional beliefs in OCD suggests that dysfunctional beliefs are associated with the cause rather than consequence of OCD symptoms (e.g., Taylor et al., 2012; Taylor \& Jang, 2011). Dysfunctional belief scores are correlated with OCD symptom severity in clinical and non-clinical samples (Abramowitz, Lackey, \& Wheaton, 2009; OCCWG, 2005; Taylor et al., 2012). Twin studies suggest a shared genetic and environmental influence between dysfunctional beliefs and OCD symptoms (Taylor \& Jang, 2011). Importantly, much like the neurocognitive findings presented above, relatives of OCD probands show higher levels of dysfunctional beliefs than do relatives of controls (Rector, Cassin, Richter, \& Burroughs, 2009). Furthermore, in keeping with the mediational model of an 
endophenotype, reduction in dysfunctional beliefs (the endophenotype) through cognitive restructuring has been found to reduce OCD symptoms (Woody, Whitall, \& McLean, 2011); additionally, changes in dysfunctional beliefs precede change in OCD symptoms (Wilhelm, Berman, Keshaviah, Schwartz, \& Steketee, 2015). Similarly, changes in dysfunctional beliefs during the first six weeks of treatment predict OCD treatment response (Diedrich et al., 2016).

While there has been much work to understand genetic factors (e.g.,Mattheisen et al., 2015; Stewart et al., 2012), there is increasing research examining other heritable factors which are also, in part, genetically determined. For example, shared personality factors (e.g., neuroticism) may explain some of the familial loading for OCD. OCD patients score higher on neuroticism than controls, as do their first degree relatives (Samuels et al., 2000). Dysfunctional beliefs may also contribute to the high familial loading for OCD, as research suggests that first degree relatives of OCD patients also have higher levels of dysfunctional beliefs (Rector et al., 2009).

Additionally, there has been some preliminary research to suggest a relationship between dysfunctional beliefs and performance on neurocognitive tasks, such that those who endorsed OCD dysfunctional beliefs, compared to those with OCD who did not endorse dysfunctional beliefs, performed worse on the Wisconsin Card Sorting Test (Bradbury, Cassin, \& Rector, 2011). Moreover, perfectionism has been shown to be associated with attention, executive function and working memory, such that those high in negative perfectionism (defined as a drive to avoid negative consequences) do less well on tests of attention and executive function, while individuals who are high on positive perfectionism (defined as a drive for success) show superior performance on working memory tasks (Slade, Coppel, \& Townes, 2009). Additionally, 
perfectionism examined uni-dimensionally was associated with less risky decision-making in OCD (Boisseau, Thompson-Brenner, Pratt, Farchione, \& Barlow, 2013).

Six categories of dysfunctional beliefs have been reliably linked to OCD symptoms; inflated personal responsibility, overestimation of threat, perfectionism, IU, overimportance of thoughts, and need to control thoughts (e.g., Clark, 2004; Frost \& Steketee, 2002; Salkovskis, 1996; OCCWG, 1997; OCCWG, 2003). Inflated personal responsibility is the belief that one is able, and has the duty to prevent, negative outcomes; overestimation of threat is the belief that negative events will occur and that their occurrence would be catastrophic; perfectionism is the belief that mistakes are intolerable/ unacceptable; IU is negative emotional response to uncertain situations; overimportance of thoughts is the belief that having a thought is significant, or that thinking a thought makes it more likely to occur; need to control thoughts is the belief that thoughts can, and should, be controlled (e.g., Clark, 2004; Frost \& Steketee, 2002; Salkovskis, 1996; OCCWG, 1997; OCCWG, 2005; Taylor et al., 2010).

Dysfunctional beliefs in OCD have largely been examined using the Obsessive Beliefs Questionnaire, either the long-form (Obsessive Compulsive Cognitions Working Group, 1997) or the short-form (OCCWG, 2005) version, which both measure the six aforementioned categories of dysfunctional beliefs. The factor structure of the OBQ short-form that has been most reliably replicated consists of three factors; inflated responsibility and overestimation of threat (RT), perfectionism and intolerance of uncertainty (PC), and overimportance and need to control one's thoughts (ICT) (OCCWG, 2003).

Research using the OBQ (either the long or short form) has examined how these belief categories predict OCD symptoms. Structural equation modeling found that RT predicted all six of the main types of OCD symptoms (checking, hoarding, neutralizing, obsessing, ordering, and 
washing) in a large nonclinical sample (Taylor et al., 2010). RT has most often been associated with washing or checking compulsions in both nonclinical (Abramowitz et al., 2009; Myers, Fisher, \& Wells, 2008; Tolin, Woods et al., 2003) and clinical samples (Ecker \& Gönner, 2008).

Structural equation modeling found that PC predicted ordering compulsions in a large nonclinical sample (Taylor et al., 2010). Indeed, PC is most commonly linked to checking or ordering compulsions in both clinical (OCCWG, 2005; Julien et al., 2008; Tolin, Abramowitz, Brigidi, \& Foa, 2003; Tolin, Brady \& Hannan, 2008) and nonclinical (Abramowitz et al., 2009; Myers et al., 2008; Tolin, Woods et al., 2003) samples. One hypothesis is that these rituals (checking and ordering) are performed to gain a sense of certainty (e.g., Carr, 1974), while others have suggested that the relationship between ordering and PC is driven by perfectionistic beliefs about the need to perfectly perform (Frost \& Steketee, 2002).

ICT examined using structural equation modeling found that ICT predicted obsessing, neutralizing, and washing compulsions in a large nonclinical sample (Taylor et al., 2010). ICT has been most often associated with obsessing in both nonclincal (Abramowitz et al., 2009; Myers et al., 2008) and clinical samples (Julien et al., 2008; Tolin et al., 2008). Examining the theoretical basis of this association, researchers have hypothesized that neutralizing is often a cognitive ritual to undo thoughts (Frost \& Steketee, 2002), which may be more common in individuals that attach undo importance to unwanted thoughts (Taylor et al., 2010). The association between ICT and washing compulsions is consistent with research finding that the experimental induction of unwanted thoughts increased the urge to wash (Zhong \& Liljenquist, 2006).

Given the largely agreed upon etiologic importance in OCD, these are the dysfunctional belief CEs that will be examined across the OCRDs in the current study. The specificity of these 
belief domains across the OCRDs has yet to be established. If a subset of dysfunctional beliefs are a broad factor, they may help explain shared pathology, comorbidity, and treatment overlap among empirically supported treatments of OCRDs (Kreuger \& Eaton, 2015). Research using the OBQ has found that some belief domains appear to be specific to OCD (e.g., need to control one's thoughts), while others may be general factors associated with a variety of psychopathology (e.g., threat estimation and perfectionism) (Tolin et al., 2003).

Importantly, it is widely accepted that perfectionism is a multidimensional construct (e.g., Frost, Marten, Lahart, \& Rosenblate, 1990; Hewitt \& Flett, 1991). As such, perfectionism has been measured using a variety of instruments, with upwards of 15 self-report measures available (Stairs, Smith, Zapolski, Combs, \& Settles, 2012). The Frost Multidimensional Perfectionism Scale (FMPS; Frost et al., 1990) is one of the most widely used self-report measures of perfectionism. The FMPS has six subscales: concern over mistakes, doubts about actions, personal standards, parental expectations, parental criticism, and organization. Research using the FMPS has also shown an association with OCD (Frost et al., 1990). Total perfectionism, concern over mistakes, and doubts about actions were found to be elevated in OCD patients compared to controls (Frost and Steketee, 1997). Similarly, concern over mistakes was elevated in OCD patients compared to controls, and doubts about actions was also elevated in OCD patients compared to controls and depressed patients (Sassaroli, 2008).

Similarly, IU is a multidimensional construct measured using several scales. The most commonly utilized scale, the Intolerance of Uncertainty Scale, has both a long and short form version (IUS; Buhr \& Dugas, 2002; Freeston, Rheaume, Letarte, Dugas, \& Ladouceur, 1994). The IUS is a self-report measure used to assess how individuals tolerate uncertainty and ambiguous situations. While there has been some disagreement about factor structure, the 
strongest empirical support is for Sexton and Dugas's (2009) two-factor solution: Factor I 'Uncertainty Has Negative Behavioral and Self-Referent Implications' and Factor II 'Uncertainty Is Unfair and Spoils Everything.' Historically, this construct has been associated with generalized anxiety disorder (e.g., Dugas, Freeston, \& Ladouceur, 1997); however, recent research suggests IU may be a common factor across psychopathology (Boswell, ThompsonHollands, Farchione, \& Barlow, 2013) including, but not limited to, social anxiety disorder (Boelen and Reijntes, 2009), major depressive disorder (e.g., Yook, Kim, Suh, \& Lee, 2010), and OCD (Tolin et al., 2003). Research using the long-form of the IUS found that checking and repeating compulsions were associated with IUC (Tolin et al., 2003). IU measured using the IUS was found to fully mediate relationship between OCD and perfectionism (measured using the FMPS) (Reuther et al., 2013). Thus, it is possible that IUS may be implicated more closely with OCD than has historically been examined.

Research examining dysfunctional beliefs across the spectrum is not as well developed as in the OCD literature. Nevertheless, some similar themes have emerged. Research examining the six aforementioned belief domains across the OCRDs is reviewed below.

Perfectionism has also been associated with HD, GDs, and BDD. It is theorized that individuals with HD are overly inclusive when determining what is important to remember due to perfectionism (Frost \& Hartl, 1996). Research using the OBQ in hoarding or hoarding symptoms found a relationship between hoarding symptoms and PC (Myers et al., 2008). Further research supports this hypothesis, as PC beliefs were predictive of hoarding symptoms (Tolin et al., 2008). Furthermore, perfectionism (measured with the OBQ) was a significant predictor of negative treatment outcome in $\mathrm{HD}$ following $\mathrm{CBT}$, above and beyond pre-treatment symptom 
severity, such that higher levels of perfectionism were associated with non-response to CBT (Muroff, Steketee, Frost, \& Tolin, 2014).

Examination of dysfunctional beliefs in the GDs similarly implicated perfectionism (O’Connor et al., 2002). Rehm, Nedeljkovis, Thomas, and Moulding (2015) found that perfectionistic beliefs contributed to the onset and maintenance of hair pulling in TTM. An examination using the long-form $\mathrm{OBQ}$ found a significant relationship between perfectionism and TTM in an Italian sample (Bottesi et al., 2016). Furthermore, perfectionism has been intimately linked to the development and maintenance of BDD, in which individuals become extremely distressed by perceived physical flaws due to rigid perfectionistic thinking (Veale, 2004; Wilhelm, 2006). In keeping with this hypothesis, several studies showing higher levels of perfectionism in BDD patients relative to healthy controls using the FMPS (Buhlmann, Etcoff, \& Wilhelm, 2008) and a subscale of perfectionism from an eating disorder questionnaire (Schieber, Kollei, deZwann, Muller, \& Martin, 2013), related to both body/appearance, as well as general trait levels of perfectionism. For a review of perfectionism in OCRDs see Pinto et al. (2017). While perfectionism is theoretically and anecdotally linked to HD and the GDs, and there has been some research in the area, additional empirical research is necessary.

IU has also been associated with hoarding symptoms. Frost and Hartl (1996) reported that the most common reason cited by those with HD for having difficulty discarding items was the idea that they could be needed at a future time. Thus, the phenomenology of HD suggests IU may be an important construct in the development and maintenance of hoarding symptoms, such that those high in IU have difficulty tolerating the uncertainty of not knowing if something will be needed in the future and therefore do not discard items. Two studies that examined IU in hoarding using the IUS (either the long or short form) found that IU predicted hoarding 
symptoms when controlling for other covariates (e.g., hoarding-related beliefs; Oglesby et al., 2013; Wheaton, Abramowitz, Jacoby, Zwerling, \& Rodriguez, 2016). Additionally, comparison of those with HD to those with OCD, GAD, or healthy controls revealed that those with HD had significantly higher levels of IU compared to controls, and levels of IU comparable and not significantly different from those with OCD or GAD, although those with GAD had the highest levels (Wheaton et al., 2016). To the author's knowledge, IU has not been systematically examined in the other OCRDs.

Research examining RT and ICT in the related disorders is scarce. One study using the OBQ found that those with HD scored higher than controls on the OBQ and did not differ significantly from those with OCD on total score or subscales; however, that was no longer the case when OCD symptoms, mood, and hoarding behaviour were controlled for (Steketee, Frost, \& Kyrios, 2003). Additionally, overestimation of threat measured using the OBQ was found to be associated with HD symptoms in some, but not all studies (Julien et al., 2008; Abramowitz et al., 2009). Preliminary work also suggests an association between overestimation of threat and control of thoughts and TTM (Bottesi et al., 2016).

To summarize, the degree to which specific dysfunctional beliefs are narrow versus broad CEs is an area for future research. Perfectionism has been implicated across the OCRDs and, indeed, across a variety of psychopathology. IU has been implicated in OCD and HD, while less is known about the relationship between IUC and the GDs. The specificity of RT and ICT remain to be seen. It is likely that the other OCRDs have narrow (disorder specific) dysfunctional beliefs, as well. For example, beliefs about emotional attachment to possessions, memory for possessions, control over possessions, and responsibility for possessions may be dysfunctional beliefs unique to HD (Steketee et al., 2003). The current dissertation will examine six 
dysfunctional beliefs implicated in OCD across the spectrum, using the OBQ short-form (OBQ; Obsessive-Compulsive Working Group, 2003), the Intolerance of Uncertainty Scale long-form (IUS; Buhr \& Dugas, 2002), and the Multidimensional Perfectionism Scale (MPS-F; Frost et al., 1990). The inclusion of the multi-method assessment of perfectionism and IU comes from research indicating that these are not unitary constructs (e.g., Frost et al., 1990; Hewitt \& Flett, 1991; Lauriola et al., 2018). Given the preliminary evidence suggestive of these dysfunctional beliefs being broad etiological factors, these constructs were assessed in more depth.

Thus, in the current study, perfectionism and IC were examined multi-dimensionally, using several self-report measures. The importance of multi-dimensionally examining constructs is highlighted as it measures several factors of the construct to ensure aspects are not missed (Rasmussen, Brown, Steketee, \& Barlow, 2013). The recommendation of administering multiple tests to measure a multidimensional construct within a study has been emphasized by several reviews (Abramovitch et al., 2015; Lezak et al., 2012).

\section{Associated Constructs}

\section{Impulsivity}

Impulsivity is another transdiagnostic construct with hypothesized importance in the

OCRDs. Behavioural genetics and neuroimaging studies suggest that impulsivity is linked to dysregulation of fronto-striatal circuits (e.g., Fineberg et al., 2010; Van de Hueval, Van de Werf, Verhoef, deWit, \& Berendse, 2010) and is impacted by dopamine and serotonin neurotransmission (Evenden, 1999; Robbins, Gillan, Smith, deWit, \& Ershe, 2012). Additionally, twin studies suggest a genetic component to impulsivity, with heritability estimates converging around .45, indicating an additive genetic effect (Livesley, Jang, \& Vernon, 1998; Pedersen, Plomin, McClearn, \& Friberg, 1988; Scroczynski, Bergeman, \& Coccaro, 1999). 
Excessive impulsivity is a shared component among many psychiatric disorders, including attention deficit hyperactivity disorder (ADHD), substance use disorders, and personality disorders (e.g., Fineberg et al., 2010; Robbins et al., 2012). Impulsivity and the GDs have a welldocumented theoretical association, so much so that prior to the release of DSM-5, TTM was classified in the DSM-IV-TR under impulse-control disorders not elsewhere classified, and ED was listed as an example of an impulse-control disorder not otherwise specified (DSM-IV, APA, 2000). Thus, impulsivity has been posited to be a CE in a variety of disorders (Eisenberg et al., 2007; Congdon \& Canli, 2005; Lombardo et al., 2012).

Impulsivity is defined as "a range of actions that are poorly conceived, prematurely expressed, unduly risky, or inappropriate to the situation and that often result in undesirable consequences" (Daruna \& Barnes, 1993, p. 23). Part of the difficulty examining impulsivity, is that, much like the aforementioned dysfunction beliefs (e.g., perfectionism), it is not a unitary construct (Evenden, 1999). Thus, a variety of definitions and measurement have been employed, including behavioural inhibition (e.g., GoNoGo task, stop-signal), interference control (e.g., Stroop), response to punishment, and self-report (e.g., Barrett Impulsivity Scale) (Nigg, 2000). One method of measurement has been the self-report Barrett Impulsivity Scale-Version 11 (BIS-11; Patton, Stanford, \& Barratt, 1995). Of note, this scale has three subscales to reflect the multidimensional construct; motor impulsivity (act without forethought/inability to withhold a response), non-planning impulsivity (lack of planning), and cognitive impulsivity (difficulty paying attention). This measure has been described as "one of the most comprehensive and accepted models of impulsivity....based on the integration of medical, behavioural, psychological, and social models" (Rasmussen et al., 2013, p. 184). Out of the subscales of the 
BIS-11, motor impulsivity, is the only factor that maps on to the traditionally used definition of impulsivity defined above (Abramovitch \& McKay, 2016).

Historically, impulsivity and compulsivity (defined as "performance of repetitive and functionally impairing overt or covert behavior without adaptive function, performed in a habitual or stereotyped fashion, either according to rigid rules or as a means of avoiding perceived negative consequences" (Fineberg et al., 2014, pg. 2) have been thought to be opposing constructs; however, recent research suggests that impulsivity and compulsivity may be best conceptualized as orthogonal constructs. This distinction between impulsivity and compulsivity is highlighted (Abramovitch \& McKay, 2016, p. 395) as the:

early notion that OCD has been associated with behavioral impulsivity has been largely abandoned. This was primarily due to the work of Hollander (2005), outlining an impulsive-compulsive continuum supported by converging pharmacological, behavioral, and neurobiological findings pertaining to differences between impulsiveness and compulsivity (Hollander, 2005).

Thus, the findings that OCD patients score significantly higher than controls on the BIS-11 total score and cognitive impulsivity subscale (Boisseau et al., 2013; Stein, Hollander, Simeon, \& Cohen, 1994; Summerfeldt, Hood, Antony, Richter, \& Swinson, 2004), are thought to be an artifact of difficulties concentrating due to intrusive thoughts and attempts to control intrusive thoughts (Abramovitch \& McKay, 2016). This contemporary conceptualization aligns with recent research indicating that $\mathrm{OCD}$ symptoms are more related to compulsivity than impulsivity (Chamberlain, Leppink, Redden, \& Grant, 2016). While behavioural impulsivity may not be implicated in contemporary models of OCD, examination in the OCRDS reveals that impulsivity may, in fact, be of etiological importance in the related disorders. 
The relationship between impulsivity and HD has received some attention in the literature, largely due to the shared phenomenology of HD with impulse control disorders, particularly the symptoms of excessive acquisition (Steketee \& Frost, 2003). A study examining acquisition impulse control problems in those with HD (e.g., acquiring free items, buying, kleptomania) found that $78 \%$ of the sample met criteria for impulse control related difficulties. Examination of self-report impulsivity (measured using the BIS-11 or the UPPS Impulsive Behavior Scale; Whiteside \& Lynam, 2001) found that impulsivity predicted hoarding symptoms in non-clinical samples (Timpano et al., 2013). However, in a clinical sample, age accounted for the relationship between HD symptoms and impulsivity (impulsivity was measured using the UPPS Impulsive Behaviour Scale and neurocognitive tasks) (Rasmussen et al., 2013). Thus, the relationship between impulsivity and HD remains an empirical question.

As aforementioned, previous classification of the GDs has implicated impulsivity (e.g., impulse-control disorders not elsewhere classified; DSM-IV, APA, 2000). A review of genetic and neurobiological data suggests that impulsivity and compulsivity are important in the etiology of TTM (Flessner, Knopik, \& McGeary, 2012). In TTM, two types of hair pulling have been consistently identified; automatic pulling (outside one's awareness) and focused pulling (pulling in response to an urge) (Flessner \& Woods, 2006; Flessner et al., 2008; Flessner et al., 2009). Impulsivity has been identified as an important construct in focused pulling (Flessner, Knopik, \& McGeary, 2012), akin to efforts to obtain pleasure/gratification as seen in other impulse-control disorders (e.g., pathological gambling, substance-related disorders) (Grant et al., 2007; Lange, Kampov-Polevoy, \& Garbutt, 2010). Examination of facets of impulsivity in TTM using the BIS-11 revealed that cognitive impulsivity (Adams, 2012; Hayes et al., 2009) and motor impulsivity (Hayes et al., 2009) predicted ED symptom severity. 
Thus, impulsivity in the current study will be examined multi-dimensionally. Many of the aforementioned neuropsychological tasks tap into various elements of impulsivity (e.g., stopsignal measuring behavioural dyscontrol). In addition to neuropsychological tasks, the current study will examine impulsivity using the BIS-11 self-report measure. The importance of multidimensionally examining constructs is highlighted, as it measures several factors of the construct to ensure aspects are not missed (Rasmussen et al., 2013), e.g., non-planning impulsivity and cognitive impulsivity. In particular, there have been discrepant results among the subscales, highlighting the importance of measuring this construct multi-dimensionally.

\section{Neurocognition, Functional Impairment, and QOL}

As outlined above, the OCRDs are associated with substantial distress and impairment (American Psychiatric Association, 2013). A British epidemiological investigation conducted by Torres et al. (2006) determined that individuals with OCD had higher levels of unemployment, lower occupational and social status, lower income, and were less likely to be married than individuals with generalized anxiety disorder, depressive episodes, or panic disorder who were matched on education level. Examination of work performance revealed similar findings, with $77.2 \%$ of individuals with OCD indicating that their ability to work was impeded (Sorensen et al., 2004).

Examination of QOL and functional impairment in HD is very much in its infancy; the limited research to date suggests that those with HD report poorer subjective and objective QOL compared to OCD patients (Saxena et al., 2011), including feeling less safe, being more victimized by crime, and being less satisfied with their living arrangements. Additionally, those with HD display worse overall psychosocial functioning (Chakraborty et al., 2012; Matsunaga, Hayashida, \& Kiriike, 2010) and greater work disability (Diefenbach, DiMauro, Frost, Steketee, 
\& Tolin, 2013; Landau et al., 2011; Pertusa, Fullana, \& Singh, 2008) than controls, and greater family impairment compared to those with anxiety disorders (e.g., generalized anxiety disorder, panic disorder) or community controls (Tolin et al., 2011).

Preliminary research suggests that individuals with TTM and ED experience an overall decrease in QOL (Odlaug \& Grant, 2008a). Social and interpersonal functioning are particularly affected in TTM and ED, with deficits in the moderate to severe range (Flessner et al., 2009; Odlaug \& Grant, 2008a). Research examining QOL and functional impairment in BDD reveals chronic impairment across various domains (Phillips et al., 2005), specifically high levels of unemployment (Didie, Menard, Stern, \& Phillips, 2008; Phillips et al., 2005) and significant social impairment (Phillips et al., 2005, Veale et al., 1996).

To date, no studies to the author's knowledge have examined whether neurocognition may be related to disability and QOL in individuals with OCD or related disorders. However, literature in other disorders, namely schizophrenia, suggest that neurocognition (specifically working memory, verbal memory, and executive functioning) may be predictive of aspects of QOL (Green, Kern, \& Braff, 2000). Similarly, a study examining neurocognition as a predictor of QOL in chronic mental illness found that memory, executive functioning and motor skills were predictive of QOL domains (Fujii, Wylie, \& Nathan, 2004). Given these findings, and research indicating that neuropsychological performance predicts functional capacity, which in turn predicts daily functioning in other psychiatric conditions (Bowie, Reichenberg, Patterson, Heaton, \& Harvey, 2006), examination of the relationship between neurocognition and QOL in the OCRDs is warranted. 


\section{Current Study}

The current dissertation examines promising CEs (neurocognitive functioning and dysfunctional beliefs) across the OCRDs. Specifically, the present work will examine neurocognitive and dysfunctional belief CEs in OCD, HD, TTM, and ED compared to nonpsychiatric controls. A BDD group was not included in this study due to lack of access to this clinical population. In exploratory analysis, this dissertation also examines CEs as predictors of QOL, and examines several CEs known to be multidimensional constructs (e.g., perfectionism, IU, impulsivity) using multiple measures.

As aforementioned, two of the theoretical perspectives with robust empirical support from which the search for CEs has stemmed, include the coriticostriatal-thalamic model (see Menzies et al., 2008 for a review) and the learning model (Clark, 2004; Frost \& Steketee, 2002; Rachman, 1997). The current study examines CEs from both aforementioned theoretical perspectives. These etiological models have produced a significant number of CEs, the inclusion of which in the current study was not feasible. For a review of neurotransmitter, EEG, and neuroimaging candidate endophenotypes in OCD, see Taylor (2012). Thus, the current study focused specifically on neurocognitive and dysfunctional beliefs due to their robustness as endophenotypes in $\mathrm{OCD}$, their potential translatability to clinical use, and the limited data regarding these CEs across the OCRDs.

Examination of candidate endophenotypes across the OCRDs in the same study allows for preliminary investigation of how narrow or broad candidate endophenotypes are. DSM-5's conceptualization of the category of OCRDs suggests that conditions in this category are distinct, but etiologically related. Thus, it could be expected that disorders within the OCRD category would share more general etiological factors, and thus more general CEs, than other disorders. 
While there has been some suggestion of specific endophenotypes (e.g., set-shifting in OCD but not TTM, Chamberlain et al., 2006), examination of the specificity of endophenotypes is in its infancy. The importance of identifying broad versus specific candidate endophenotypes is highlighted in a review of OCD endophenotypes by Taylor (2012) which states to:

facilitate research into the etiology of OCD and other disorders, it is important to determine whether a given $[\mathrm{CE}]$ is most useful for studying etiologic factors specific to OCD or a particular type of OCD symptoms, or whether the [CE] is more useful for understanding broad-spectrum etiologic factors that shape many different kinds of psychopathology. (p. 260)

Similarly, the aforementioned review (Taylor, 2012) highlighted a gap in endophenotype literature that resulted from studying OCD in isolation from other disorders and spoke of the need to study OCD in relation to other disorders in order to identify broad etiologic factors.

While there has been considerable research examining neurocognitive functioning as a $\mathrm{CE}$ in $\mathrm{OCD}$, the examination in the related disorders is relatively novel, particularly for the grooming disorders. A seminal review of endophenotypes in OCD highlights the necessity of identifying many CEs to fully understand complex disorders (Taylor, 2012). Thus, in addition to examining existing theoretically established CEs, the current dissertation seeks to identify new endophenotypes. Specifically, dysfunctional beliefs known to be important in OCD (e.g., perfectionism) will be examined in the other OCRDs and impulsivity will be examined across the spectrum. The current dissertation also examines several CEs known to be multidimensional constructs (e.g., perfectionism, IU, impulsivity) using multiple measures. The importance of examining multidimensional constructs has been emphasized in several reviews to ensure aspects 
of the construct are not missed (e.g., Abramovitch et al., 2015; Lezak et al., 2012; Rasmussen et al., 2013).

Recommendations made in critical reviews were incorporated, where possible, into the current research design. The current study sought to address the limitations of previous research by addressing multiple comparisons using the Holm procedure (Holm, 1979), reporting symptom severity, measuring and statistically controlling for depressive symptoms, reporting age of onset, reporting recruitment source, and reporting education level. Nevertheless, the current study suffered from several common limitations in this line of work (e.g., limited power and inflated type 1 error despite correction for multiple comparison) that are highlighted throughout the results and discussion session. The power limitations of this study are such that there is an inherent bias toward affirming the null hypothesis. Thus, a non-significant finding could mean either: 1) that there is no effect; or 2) that the study was too underpowered to detect significant group differences, particularly for small effects (Cohen, 1962). This limitation is stressed here to allow the reader to interpret the results accordingly. It is hoped that this work will serve as pilot work, and that null findings will not deter future research.

The current dissertation aimed to address the following questions about CEs in the OCRDs: (1) What is the specificity of neurocognitive CEs across the OCRDs? What is the specificity of dysfunctional belief CEs across the OCRDs? (2) Is impulsivity correlated with the OCRDs? (3) What are the correlations between CEs and QOL? The following general hypotheses follow; 1) It is predicted that there are general endophenotypes underlying all of the OCRDs, and that there are some specific CEs linked to some OCRDs but not others; 2) 
Impulsivity will be associated with the related disorders, but not OCD; 3) CEs are associated with impairments in QOL. ${ }^{2}$

\section{Clinical Implications}

With endophenotypes increasingly recognized as both a valid and important approach to the understanding of complex disorders, a clearer understanding of CEs in the OCRDs may yield information about diagnostic classification, etiology and course. From an etiologic standpoint, examination of $\mathrm{CE}$ has the potential to facilitate the detection of genetic and environmental factors that contribute to disorder development and maintenance.

As aforementioned, CEs have implications for diagnostic classification. Diagnostic classification is particularly relevant given the recent reclassification of the OCRDs, and the controversy surrounding this reclassification (e.g., Bartz \& Hollander, 2006; Brakoulias et al., 2011; Mataix-Cols, Rosario-Campos, \& Leckman, 2005) in DSM-5. Some have suggested that an etiological-based disorder classification is an alternative to the current symptom-based classification currently utilized (e.g., Taylor et al., 2010). The classification of disorders is important, as the "categorization [of disorders] may well influence conceptualization of disorders, and ultimately potentially the way in which they are assessed and treated" (Stein et al., 2010, p. 496). For example, should general dysfunctional belief CEs be implicated across disorders, psychotherapy groups may be more likely to be transdiagnostic (e.g., Barlow, SauerZavala, \& Ellard, 2010, Unified Protocol for Transdiagnostic Treatment of Emotional Disorders), which has significant implications for treatment delivery, including accessibility and wait-times.

\footnotetext{
${ }^{2}$ The current dissertation could not test more specific a priori hypotheses due to the limitations of the current empirical literature. Specifically, as aforementioned in the literature review, the current literature base is not reliable enough or developed adequately to make precise predictions.
} 
Additionally, a better understanding of CEs in the OCRDs could lead to novel treatment targets. As CEs arise from etiological conceptual models (biological and cognitive), these endophenotypes could be areas for intervention. As aforementioned, the concept of endophenotypes has been expanded to include being a vulnerability factor (e.g., Taylor, 2012). The mediating variable model suggests that treatment could target the endophenotype, such that reducing levels of the endophenotype would lower the risk of psychopathology (Kendler \& Neale, 2010). Thus, if a candidate endophenotype is a risk factor, identification of candidate endophenotypes could contribute to prevention or early intervention work.

One such additional area of intervention arising from the biological etiological model includes cognitive remediation, which is a method of rehabilitating neurocognitive deficits. Although research into cognitive remediation in OCRDs is currently sparse, literature in other psychiatric disorders, particularly schizophrenia, has revealed significantly improved performance on the WCST with a large mean effect size (Cohen's $d=0.96$ ) following cognitive remediation (see Kurtz, Moberg, Gur, \& Gur, 2001 for a review). Similarly, preliminary research utilizing cognitive remediation in those with HD demonstrated a significant improvement in attention relative to a relaxation control condition following 24 sessions of cognitive remediation; however, no significant improvement emerged in memory, executive functioning, or hoarding severity (DiMauro, Genova, Tolin, \& Kurtz, 2014). Examination of cognitive remediation as an adjunct to behaviour therapy in a geriatric hoarding sample revealed significant reductions in hoarding severity, with a doubling of previously reported response rates, suggesting that cognitive rehabilitation with exposure therapy is a promising approach to treatment (Ayers et al., 2014). 
Similarly, an area of intervention arising from the cognitive model is the modification of maladaptive dysfunctional beliefs. Cognitive restructuring is already an important treatment component in the first-line cognitive-behavioural treatment of all of the OCRDs (e.g., Steketee et al., 2010; Teng, Woods, \& Twohig, 2006; van Minnen, Hoogduin, Keijsers, Hellenbrand, \& Hendriks, 2003; Wilhelm, Philips, Fama, Greenberg, \& Steketee, 2011). Examination of broad versus specific dysfunctional belief endophenotypes can assist clinicians in identifying and modifying these belief domains.

\section{Method}

\section{Participants}

A total of 77 participants took part in this study, divided into four groups; OCD $(n=21)$, HD $(n=16)$, Grooming Disorders (GD) which included both TTM and ED $(n=18)$, and control participants $(n=22)$. The final sample was $65 \%$ female, with a mean age of 39 years old $(\mathrm{SD}=$ 14.2). A BDD group was not included because of difficulties recruiting this clinical population due to low rates of treatment seeking behaviour (Conroy et al., 2008). Collapsing TTM and ED into GD is justified given their phenomenological similarities (e.g., Lochner et al., 2002; Odlaug \& Grant, 2008a,b), and the suggestion that TTM and ED have more similarities with each other than other OCRDs (e.g., Snorrason et al., 2012). Indeed, in the first twin study to include all of the OCRDs in the same analyses, one factor emerged that was exclusively related to TTM and ED, suggesting that these disorders may, in fact, be different phenotypic expressions of the same disorder (Monzani et al., 2014). Thus, many studies have routinely collapsed the TTM and ED groups into a general GD group for analysis (Flessner et al., 2009; Grant \& Christenson, 2007; Hanna et al., 2005). 
Patients were recruited from the Frederick W. Thompson Anxiety Disorders Centre at Sunnybrook Health Sciences Centre in Toronto, Canada, or were recruited from the community using flyers and online advertisements. Control participants were recruited from the community or from a postsecondary institution in Toronto, Canada. The breakdown of recruitment sources is as follows: Frederick W. Thompson Anxiety Disorders Centre at Sunnybrook Health Sciences Centre (clinical $n=44[\mathrm{OCD}=14, \mathrm{HD}=15, \mathrm{GD}=15]$, control $n=0$ ); Community (clinical $n=$ $10[\mathrm{OCD}=7, \mathrm{HD}=1, \mathrm{GD}=3]$, control $n=18$ ); and Ryerson University (clinical $n=1[\mathrm{OCD}=1$, $\mathrm{HD}=0, \mathrm{GD}=0]$, control $n=4)$. The Frederick W. Thompson Anxiety Disorders Centre at Sunnybrook Health Sciences Centre is an outpatient, specialty clinic that offers specific expertise in the treatment of OCRDs. A referral to the Frederick W. Thompson Anxiety Disorders Centre requires a referral from a family physician or psychiatrist. At the time of recruitment, referral criteria for this tertiary care, specialty clinic were as follows: patients must have a principal diagnosis of an OCRD and are either seeking diagnostic clarification, treatment recommendations, or evidence-based treatment including group CBT.

Participants who saw the advertising for the current study and were interested contacted the research team for an eligibility screen. Potential participants were screened using a telephone interview to determine eligibility. Inclusion criteria included 1) Principal diagnosis of OCD, HD or GD, or no Axis I diagnosis for a healthy control; 2) Minimum symptom duration of one-year for patients; 3) Aged $18-65$; 4) Ability to give informed consent; and 5) Stability on psychotropic medications for at least six weeks prior to participation for patients. Principal diagnosis was assessed by the Structured Clinical Interview-IV for the Diagnostic and Statistical Manual of Mental Disorders (DSM)-Fourth Edition Text Revision (SCID-IV; First, Spitzer, \& Gibbon, 1996) with an OCRDs insert assessing for DSM-5 criteria. This insert was created by 
one of the co-authors (PR), due to the fact that the available clinical interviews at the time of data collection did not assess the obsessive-compulsive spectrum; indeed, no gold standard instrument for assessing the related disorders was available prior to the Structured Clinical Interview for the Diagnostic and Statistical Manual of Mental Disorders (DSM)-Fifth Edition (SCID-5) (2016). Healthy control participants were included if they were free from any Axis 1 disorder.

Exclusion criteria included 1) not being fluent in English; 2) colour blindness; 3) comorbid schizophrenia, bipolar disorder, psychosis, or substance use disorders; and 4) past neurological disorders identified by history, such as traumatic brain injury, seizures, or stroke. Other co-morbid conditions, including depression and anxiety disorders, were not exclusionary due to the high rates of co-morbidity in this population (e.g., Ruscio et al.,2010), and the importance of generalizability of results. Individuals presenting with more than one OCRDs were grouped by primary diagnosis (diagnosis that the patient and assessing clinician believed was most currently impairing), but were not excluded from participation for aforementioned reasons (e.g., high rates of comorbidity within the OCRDs, with estimates of $67.1 \%$ having a lifetime history of at least one comorbid OCRD; Lochner et al., 2014). Participants were financially compensated for their participation. This study was approved by the Research Ethics Boards at Ryerson University and Sunnybrook Health Sciences Centre.

\section{Power}

A power analysis for the current study was complicated by 1) the use of both parametric and nonparametric statistics; 2) the use of the Holm's procedure (described below) that alters alpha so the nominal $p$ value for some analyses were smaller than .05 (as low as $p=.008$ for one analysis); and 3) the differing statistical tests utilized (correlation, $t$-test, ANOVA, chi square). Thus, the power analysis presented below does not apply to all (or most) analyses. Nevertheless, 
in an attempt to demonstrate one of the significant limitations of this study, a power analysis was conducted for ANOVA, based on the smallest effect size anticipated in the extant literature. According to a meta-analysis of neuropsychology in $\mathrm{OCD}$, small effect sizes were found for verbal memory measures (Abramovitch et al., 2013). Thus, the power analysis was conducted using a small effect size $(r=.2)$ according to Cohen (1977). Power estimates were calculated using the G-power calculator. Accordingly, $n=69$ in each group were needed to achieve a small effect size $(r=.2 ; \alpha=.05$; power $=.80)$. Thus, as aforementioned and described in more detail in the limitations section, the current study was insufficiently powered to detect small effects.

\section{Measures}

Participants completed a battery of psychometrically validated measures including semistructured clinical interviews, self-report questionnaires, and neurocognitive tasks. When reviewing reliability psychometrics, Kappa values above .70 were considered to reflect good agreement; Kappa values from .50 to .70 were considered to reflect fair agreement, and Kappa values below .50 were considered to reflect poor agreement (First \& Gibbon, 2004).

\section{Diagnostic measures.}

The Structured Clinical Interview-IV for the Diagnostic and Statistical Manual of Mental Disorders (DSM)-Fourth Edition Text Revision (SCID-IV; First et al., 1996) assessed for the presence of Axis 1 DSM-IV symptoms. The SCID-IV is the gold standard diagnostic measure. It is a semi-structured, clinician-administered, diagnostic interview. The SCID-IV demonstrates adequate to fair reliability for some diagnoses of interest for this study over a 7-10 day interval; OCD $(\alpha=.60)$, Major Depressive Disorder $(\alpha=.61)$, with a range of reliability across diagnoses from $\alpha=.35$ for Dysthymic Disorder to $\alpha=.78$ for Posttraumatic Stress Disorder (Zanarini et al., 2000). Given that the SCID-IV did not assess for all of the OCRDs, an insert was included to 
assess for DSM-5 criteria of theOCRDs, which was created by Dr. Peggy Richter and Dr. Neil Rector at Sunnybrook Health Sciences Centre. The insert mapped onto DSM-5 criteria for the OCRDs. Unfortunately, psychometrics are not yet available for this insert.

\section{Symptom severity measures.}

The Yale-Brown Obsessive-Compulsive Scale (YBOCS; Goodman et al., 1989) is a semi-structured interview of OCD symptoms and severity. The YBOCS is comprised of a 74item symptom checklist, including a list of 40 obsessions and 29 compulsions. The obsessions and compulsions are categorized by content to yield a symptom checklist; cleaning/washing, checking, repeating, counting, ordering/arranging, hoarding/collecting, mental compulsions, and miscellaneous. In addition, the YBOCS contains 10 items that assess symptom severity. The severity scale assesses time spent, interference, distress, resistance, and control of symptoms. This is rated on a 5-point Likert scale from 'no symptoms' to 'extreme symptoms', which yields a total severity score ranging from 0 to 40 . Those scoring $0-7$ are sub-clinical; 8-15 are mild; 16-23 are moderate; $24-31$ are severe; and 32-40 are extreme. The YBOCS total score has fairto-good internal consistency $(\alpha=.69-.88)$, inter-rater reliability $(r=.93)$, and test-retest reliability over a range of 10-103 days ( $r=.61$; Goodman et al., 1989; Woody, Steketee, \& Chambless, 1995). In the current study, the YBOCS total score had good internal consistency ( $\alpha$ $=.82)$. It was not administered to controls.

The Obsessive-Compulsive Inventory-Revised (OCI-R; Foa et al., 2002) is an 18-item measure that assesses the presence and severity of common OCD symptoms. It has six subscales; washing, checking, ordering, obsessing, hoarding, and neutralizing. Responses are made on a 5point Likert scale ranging from 'not at all' to 'extremely'. Scores range from 0-72, with higher scores indicating greater OCD severity. The OCI- R demonstrates good psychometric properties, 
including good internal consistency $(\alpha=.83)$, test-retest reliability over an interval of two weeks $(\mathrm{r}=.82-.84)$, and convergent validity (Abramowitz \& Deacon, 2006; Foa et al., 2002). The recommended cut-off score is 21 , with scores at or above this level indicating the likely presence of OCD. The mean score typically reported for individuals with OCD is approximately 28.0 (SD $=13.53$ ) (Foa et al., 2002). The OCI-R total score was also computed with the hoarding items removed, denoted as OCI-OCD as per Wootton et al (2015). The OCI-OCD has a cut-score of 12 , and the mean score typically reported for individuals with OCD is approximately 23.94 (SD $=12.11$ ) (Wootton et al., 2015). Both total scores (with and without hoarding items) are included to allow for comparison to previous literature including the full scale. In the current study, the OCI-R had adequate internal consistency $(\alpha=.85 ; \alpha=.88)$ for patients and controls, respectively. The OCI-OCD also had good internal consistency $(\alpha=.89 ; \alpha=.83)$ for patients and controls, respectively.

The Savings Inventory-Revised (SI-R; Frost, Steketee, \& Grisham, 2004) is a 23-item scale used to assess the presence and severity of hoarding symptoms. Responses are made on a 5-point Likert scale, from 'none' to 'almost all/complete'. Scores range from 0-92, with higher scores indicating greater hoarding symptom severity. The scale is comprised of three subscales; difficulty discarding, excessive clutter, and compulsive acquisition. The scale demonstrates good reliability $(\alpha=0.92)$ and convergent validity, showing strong correlations with other measures of hoarding, including home-based ratings (Frost et al., 2004; Frost, Steketee, Tolin, \& Renaud, 2008). The measure has a clinical cut-off score of 41 , with scores at or above this cut-off indicating clinically significant hoarding symptoms, likely at diagnostic threshold. The mean score often reported for individuals with HD is approximately 62.0 ( $\mathrm{SD}=12.7$ ) (Frost et al., 
2004; Tolin et al., 2011). In the current study, the SI-R had good internal consistency $(\alpha=.96 ; \alpha$ $=.89$ ) for patients and controls, respectively.

The Massachusetts General Hospital Hair-Pulling Scale (MGH; Keuthen et al., 1995) is a 7-item self-report measure to assess for frequency and intensity of the urge to pull hair, time spent pulling, interference due to pulling, and associated distress and avoidance. Responses are made on a 5-point Likert scale. The scale yields a total score (range 0 to 28), with higher scores indicating increased hair-pulling frequency and severity. The MGH has good reliability $(\alpha=.82)$ and convergent and discriminant validity, and sensitivity to change with treatment (Keuthen et al., 1995). A cut-off score of 17 indicates clinically significant pulling. The mean score often reported for individuals with TTM is approximately 18 ( $\mathrm{SD}=5.1)$ (e.g., Weidt, Klaghofer, \& Kuenburg, 2015). In the current study, the MGH had adequate internal consistency $(\alpha=.95)$ for patients. It was not administered to controls.

The Skin Picking Scale (SPS; Keuthen et al., 2001) is a 6-item self-report measure to assess for the frequency and intensity of the urge to pick skin, time spent picking, interference due to picking, and associated distress and avoidance. Responses are made on a 5-point Likert scale. Total scores range from $0-24$, with higher scores indicating more severe skin picking. The SPS has good reliability $(\alpha=.80)$ and validity. A cut-off score of 7 indicates clinically significant skin picking. The mean score often reported for individuals with ED is approximately $12.74(\mathrm{SD}=3.83)$ (Keuthen et al., 2001). In the current study, the SPS had good internal consistency $(\alpha=.96)$ for patients. It was not administered to controls.

The Beck Depression Inventory Second Edition (BDI-II; Beck, Steer, \& Brown, 1996) is a 21-item self-report scale which assesses for the presence and severity of depressive symptoms. Responses are made on a Likert scale ranging from 'not at all' (0) to 'extreme' (3). Scores range 
between 0 and 63, with higher scores indicating greater depressive severity. Items 16 and 18 are rated out of seven options to assess for both increased and decreased sleep and appetite, respectively. The BDI-II shows good internal consistency $(\alpha=.92)$, one-week test-retest reliability $(r=.93)$, and convergent validity (Beck et al., 1996; Schneibel et al., 2012). The scale has clinical cut-offs as follows; 0-13: minimal depression; 14-19: mild depression; 20-28: moderate depression; and 29-63: severe depression. In the current study, the BDI-II had good internal consistency $(\alpha=.91 ; \alpha=.88)$ for patients and controls, respectively.

The Beck Anxiety Inventory (BAI; Beck, Epstein, Brown, \& Steer, 1988) is a 21 -item self-report scale which assesses for the presence and severity of anxiety symptoms. Responses are made on a 4-point Likert scale ranging from 'not at all' (0) to 'severely' (3). Scores range between 0 and 63, with higher scores indicating greater anxiety severity. The scale has clinical cut-off as follows: 0-7 indicates minimal or normal level of anxiety, 8-15 is mild anxiety, 16-25 is moderate, and 26-63 is severe. The BAI has been shown to have good internal consistency ( $\alpha$ $=.92)$, and one-week test-retest reliability $(r=.75)$. In the current study, the BAI had good internal consistency $(\alpha=.91 ; \alpha=.90)$ for patients and controls, respectively.

\section{Neurocognitive measures.}

\section{Measure of IQ.}

The Wechsler Test of Adult Reading (WTAR; Holdnack, 2001) is a screening measure to estimate premorbid intelligence (intelligence prior to the onset of the OCRDs). The participant is instructed to read a list of 50 words out loud. The dependent variable is each correct pronunciation, which is given a score of 1 . Thus, the maximum raw total score is 50 . The raw score is then standardized by age and demographics. The WTAR demonstrates good reliability 
$(\alpha=.92)$ and predictive validity $(r=.90)$, and is highly correlated with full scale IQ $(r=.73)$

(Holdnack, 2001; Spreen \& Strauss, 2006).

\section{Measure of response inhibition.}

The Stop Signal Task (SST; Logan et al., 1984) is a measure of the speed of executing and voluntarily inhibiting a motor response. There are two components to the task, a 'go' task and a 'stop' task. The 'go' task involves a simple choice reaction time task. Participants watch a computer screen, which initially presents a fixation point for $500 \mathrm{~ms}$. The 'go' task stimuli are upper case letters $\mathrm{X}$ and $\mathrm{O}$ presented in the centre of the screen for 1,000 ms, followed by a 2,000 ms blank screen. Participants respond as quickly and as accurately as possible with one of two keys, depending on which letter is presented. The 'stop' signal, which is presented intermittently following the letters, is a $1,000 \mathrm{~Hz}$ tone. When the tone is heard, the participant is instructed to inhibit pressing the letter's corresponding key. The tone occurs randomly on $25 \%$ of trials. The later the tone is presented, the more difficult it is to inhibit the response. The ability of an individual to stop depends on a 'race' between the 'go' and 'stop' processes. The 'stop' signal delay is initially set at $250 \mathrm{~ms}$, and a 'tracking' algorithm converges on the 'stop' signal delay at which individuals are able to inhibit $50 \%$ of responses. The task consists of eight blocks, each with 32 trials. Twenty-four of these trials are 'go' trials without a 'stop' signal, while eight trials include the 'stop' signal. The main dependent variable is the Stop Signal Reaction Time (SSRT), which measures the latency between the appearance of the 'stop' signal, and the initiation of the 'stop' process. This is calculated by subtracting the mean delay from the mean reaction time.

Larger latencies in SSRT (slower speed) of the stopping process represent deficits in inhibition. Other dependent variables examined in the current study included the Mean Reaction Time (MRT) which captures the latency of the 'go' process (for a review, see Logan, 1994). 


\section{Measure of interference control.}

The Stroop Colour and Word Test (Golden, 1976) assesses selective attention and cognitive flexibility. Participants are presented with one of three cards. Card 1 has the name of a colour printed in black ink (word card). Card 2 has patches of colours (colour card). Card 3 has colour names printed in another ink colour (colour-word card). Participants are asked to read the colour name for card 1 and to name the ink colour for cards 2 and 3 as quickly as they can in 45 seconds. The main dependent variable is the Stroop interference colour-word score, which is the difference between the score from the word card and the score for the colour-word card. Raw scores were standardized based on age and demographic characteristics.

\section{Measures of set-shifting.}

The Wisconsin Card Sorting Task (WCST; Berg, 1948) is a measure of mental flexibility, specifically set-shifting ability. There are four decks of cards with different colours, numbers, and shapes on the top of the screen, and one card on the bottom of the screen that changes upon each selection. The goal of this task is to select the appropriate card on the top of the screen, given which card is present at the bottom of the screen. After each selection, the computer provides feedback. The rules of similarity are not explicitly provided and change throughout the course of the task, such that the participant's task is to shift cognitive strategies with the changing similarity contingencies. The dependent variables examined from this measure include the total number of errors made on the task (errors made by not following the sorting rule), perseverative errors (the number of errors that would have been correct for the preceding rule), non-perseverative errors (the remaining incorrect responses other than the perseverative errors), the number of successful categories completed (a successful set is defined as 10 correct 
consecutive responses on a given rule), total trials administered (maximum of six), and total trials correct (total number of correct responses).

The Intradimensional/Extradimensional Task (ID/ED; Cambridge Neuropsychological Test Automated Battery CANTAB, 2006) is a computerized visual discrimination task that measures basic set-shifting (e.g., shifting from a previously rewarded response to a new response based on negative feedback). Participants are presented with two shapes and asked to select which of the shapes is correct, and are then given feedback. Once the participant learns the rule to pick the correct shape and responds correctly on six consecutive trials, the rule changes so that the other shape is correct. The dependent variables include total errors committed at each stage, and the number of stages passed.

\section{Measures of nonverbal memory.}

The Rey-Osterrieth Complex Figure Test (RCFT; Osterrieth, 1944) is a measure of visuospatial memory, working memory, and strategic learning. Participants are first asked to copy a complex figure line drawing freehand (recognition). Participants are then asked to draw the figure again from memory 3 minutes and 30 minutes after seeing the original in immediate recall and delayed recall, respectively. Accuracy is then scored following the 36-point system (Lezak, 1995). Dependent variables include copy accuracy, immediate recall accuracy, and delayed recall accuracy. RCFT data was standardized according to age and gender. Higher scores indicate better performance.

The Spatial Working Memory Task (SWM; Owen, Downes, Sahakian, Polkey, \& Robbins, 1990) is a computerized measure to assess visuospatial working memory. The participant looks for a token hidden under a box. On each trial the token will never be placed in the same box twice. The number of search locations increases across the task. Dependent 
variables include the total number of between-search errors (returning to boxes where tokens were previously found), strategy scores, and latency scores.

\section{Measures of verbal memory.}

The California Verbal Learning Test (CVLT; Delis et al., 1987) assesses verbal memory, including retention and retrieval, which also taps into strategic learning and organizational strategies. Participants are read a list of 16 words from four categories. This list is read to participants on five sequential trials, with the participant recalling as many items as possible after each administration. Following the fifth trial, the participant is given an interference list of 16 different items to recall. The participant is then asked for the short delay free recall trial, where the participant is asked to recall words from the initial list, followed by the cued recall trial where the participant is cued by category and asked to recall words from the first word list. After a 20 minute delay, the participant is asked for a long delay free and cued recall. Finally, the participant is given a list of 40 words, and asked whether or not each item was on the initial word list. Dependent variables include the total number of target items correct across learning trials 15 (the number of words correctly recalled), total free short delay recall (the number of correct words following a short delay), free recall intrusions (words recalled that were not on the list), and total repetitions (total number of times a participant repeated the same word during recall).

\section{Measures of processing speed.}

Digit Symbol-Coding (Wechsler, 1997) is a measure of cognitive processing speed.

Across the top of the page there are numbers associated with corresponding symbols. On the rest of the page, there are boxes with numbers, but the corresponding symbol is missing. Participants are instructed to copy the corresponding symbol in each box, consecutively. The dependent 
outcome is the number of correct symbols copied in 120 seconds. Higher scores indicate better performance. Incorrectly drawn symbols are subtracted from the total score.

The Trail Making Test (TMT; Army Individual Test Battery, 1994; Lezak, 1995) assesses executive functioning, including information processing speed, working memory, and taskswitching ability. In Part A of the task, participants connect the dots from 1 to 25 as quickly as they can. In Part B of the task, participants draw a line alternating between numbers and letters as quickly as they can. The dependent variable is the time it takes the participant to complete the task. Error rate is not recorded, per se. Rather, errors are accounted for by an increased completion time.

\section{Quality of life measures.}

The Quality of Life Enjoyment and Satisfaction Questionnaire- Short Form (Q-LES-QSF; Endicott, Nee, Harrison, \& Blumenthal, 1993) is a 16-item self -report measure of life enjoyment and satisfaction over the past week. Specifically, individuals rate the extent to which they are satisfied with their life and current circumstances based on physical health, mood, work, activities, relationships, functioning, sexual desire, economic status, overall well-being, and medication. Responses are made on a 5-point Likert scale from 'very poor' to 'very good'. Scores range from 16-70 (as medication and overall well-being are rated separately), with higher scores indicating better QOL. The scale has good internal consistency $(\alpha=.90)$, one-week test retest reliability ( $r=.93$ ), and convergent validity (Stevanovic, 2011). In the current study, the QLES-Q-SF had good internal consistency ( $\alpha=.83 ; \alpha=.86)$ for patients and controls, respectively.

The Sheehan Disability Scale (SDS; Sheehan, 1983) is a 5-item self-report visual analog scale that measures symptom disability and interference. The scale measures the effect of the 
patient's symptoms on work/school, social life, and family life/home responsibilities. Each subscale can be scored independently or combined to produce a total score representing global impairment. Responses are made on an 11-point visual analog scale, from 'not at all' to 'extremely'. The total score ranges from 0-30, with higher scores indicating greater impairment. The final two items assess number of days their symptoms caused work or school absence and number of days underproductive at work or school. These final two items are not included in the total score. The SDS has good reliability $(\alpha=.86)$. In the current study, the SDS had good internal consistency $(\alpha=.73 ; \alpha=.95)$ for patients and controls, respectively.

\section{Dysfunctional belief questionnaire measures.}

The Obsessional Beliefs Questionnaire (OBQ; Obsessive-Compulsive Working Group, 2003), is a 44-item self-report scale that assesses core beliefs and maladaptive appraisals that are common in OCD. The measure includes three subscales: inflated responsibility and threat overestimation; perfectionism and IU; and over importance and need to control thoughts. Responses are made on a 7-point Likert scale, from ‘disagree very much' to 'agree very much'. The OBQ has good reliability ( $\alpha$ range 0.80 - 0.96 for the subscales) and validity. In the current study, the OBQ had good internal consistency $(\alpha=.96 ; \alpha=.95)$ for patients and controls, respectively.

The Intolerance of Uncertainty Scale (IUS; Buhr \& Dugas, 2002) is a 27-item self-report measure used to assess how individuals tolerate uncertainty and ambiguous situations. Responses are made on a 5-point Likert scale, from 'not at all characteristic of me' to 'entirely characteristic of me'. Scores range from 27 to 135, with higher scores indicating greater IU. The IUS has good internal consistency $(\alpha=.94)$, test-retest reliability over a 5 week period $(r=.74)$, and convergent validity (Buhr \& Dugas, 2002; McEnvoy \& Mahoney, 2011). In the current study, the IUS had 
good internal consistency $(\alpha=.92 ; \alpha=.96)$ for patients and controls, respectively. The IUS was used as the self-reported measure of IU in this study.

The Frost Multidimensional Perfectionism Scale (MPS-F; Frost et al., 1990) is a 35-item self-report measure of perfectionism. Responses are made on a 5-point Likert scale, from 'strongly disagree' to 'strongly agree'. The MPS has six subscales; concern over mistakes, doubts about actions, personal standards, parental expectations, parental criticism, and organization. However, the organization subscale shows poor convergent validity with the other subscales. Thus, commonly, the total score is calculated using the first five subscales only, and the organization subscale is excluded (Frost et al., 1990, pp. 454-456). This was the scoring used in the current study. Additionally, the MPS-F has been criticized for factorial instability (e.g., Rheaume, Freeston, Dugas, Letarte, \& Ladouceur, 1995). The current study thus used the three factor solution developed by Stober (1998) in which parental expectations and parental criticism are combined, and concern over mistakes and doubts about action are also combined. Therefore, the total score is based on 29 items, with higher scores indicating more perfectionism. The range of total scores is $29-145$. The MPS has good reliability $(\alpha=.90)$ and validity. In the current study, the MPS-F had good internal consistency $(\alpha=.93 ; \alpha=.78)$ for patients and controls, respectively. The MPS was used as the measure of perfectionism in this study.

\section{Associated Construct Questionnaires.}

The Indecisiveness Scale (IS; Frost \& Shows, 1993) is a 15-item self-report scale that measures decision-making difficulty, specifically the tendency to postpone making decisions. Responses are made on a 5-point Likert scale, from 'strongly disagree' to 'agree'. Total scores range between 15 and 75, with higher scores indicating greater difficulty with decision-making. The IS has good reliability $(\alpha=.90)$ and validity (Frost \& Shows, 1993). In the current study, the 
IS had fair internal consistency ( $\alpha=.53 ; \alpha=.77)$ for patients and good internal consistency for controls, respectively. The internal consistency for the clinical participants is lower than in other published studies utilizing clinical groups (e.g., Yook et al., 2010).

The Barratt Impulsivity Scale Version 11 (BIS-11; Patton et al., 1995) is a 30-item selfreport measure used to assess impulsivity. Responses are made on a 4-point Likert scale; from 'rarely/never' to 'almost always/always'. The scale has three subscales: motor impulsivity, nonplanning impulsivity, and attentional impulsivity. Total scores range from 30 to 120, with higher scores indicating greater impulsivity. The BIS-11 has been shown to have good internal consistency on the total scale $(\alpha=.83)$. The BIS has also shown high concurrent validity (Stanford et al., 2009). In the current study, the BIS had good internal consistency for patients ( $\alpha$ $=.81)$ and controls $(\alpha=.88)$ on the total score. The BIS was used as the self-reported measure of impulsivity in this study.

\section{Procedure}

Participants who indicated interest in the study (via the recruitment procedure outlined above) were initially screened by telephone using a modified version of the SCID screen. The screen was modified to also screen for OCRD criteria (HDD, TTM, ED) to determine study eligibility. Written informed consent was obtained from participants prior to beginning the study protocol. Participants completed a 4.5 hour test battery that included a clinical interview, selfreport questionnaires, paper-and-pencil tasks, and computerized measures, at either Ryerson University or Sunnybrook Health Sciences Center. However, a subset of participants, $27 \%$ ( $n=$ 15), had a recent (meaning within the last twelve weeks) 1.5 hour clinical interview (SCID; YBOCS) on file at Sunnybrook Health Sciences Centre as part of their routine standard of care. For those participants with a recent clinical interview on file, the clinical interviews were not re- 
administered following neurocognitive testing to avoid redundancy and potential participant distress. Instead, the data were shared between institutions after obtaining institutional and patient consent. Thus, for the $27 \%$ of participants with recent clinical interviews on file, their study visit lasted 3 hours. In all cases, clinical interviews and neurocognitive testing were conducted by graduate students in clinical psychology or research assistants under the supervision of a licensed psychologist or psychiatrist. As part of the SCID-IV training provided at Sunnybrook, all individuals participating in administering the clinical interview process observed the SCID-IV training tapes, observed three clinical interviews, and had three clinical interviews observed, prior to conducting clinical interviews. Research suggests that, with training, novice interviewers do not significantly differ from expert raters on inter-rater reliability or diagnostic accuracy on the SCID-IV (Ventura, Liberman, Green, Shaner, \& Mintz, 1998). Inter-rater reliability statistics were not computed for this study and, as such, it is a limitation of the methodology. However, participants recruited from the Frederick W. Thompson Anxiety Disorders Centre at Sunnybrook Health Sciences Centre $(n=44)$ had a historical diagnosis of an OCRD from a family physician or psychiatrist for referral to the specialty clinic which provides anecdotal corroborating evidence of diagnostic accuracy in the current study. All participants received the measures in the same order of administration. The order of testing was carefully chosen in order to avoid interference effects. Therefore, tests measuring similar constructs were not administered in sequential order. Participants were debriefed and financially compensated following the completion of the protocol.

\section{Statistical Analyses}

Analyses were conducted using the Statistical Package for the Social Sciences (SPSS) version 21 . The dataset was screened for missing data and normality prior to evaluating study 
hypotheses. Missing questionnaire data was addressed using means replacement, which is considered to be a robust method to address item-level missing data (Dong \& Peng, 2013; Enders, 2003). This resulted in less than $1 \%$ of total data filled in through this method. For the neurocognitive measures, participants were excluded pair-wise from analyses for missing data points, but were still included in the analyses for completed tests. Therefore, the sample size varied across the neurocognitive tasks, due to missing data, outliers, as well as differing parameters around valid trials. This is described in detail for the various neurocognitive tasks in the results section.

Parametric assumptions were checked, including normality, homogeneity of variances, linearity, and independence, prior to performing parametric statistics. In the instances where assumptions were violated, data were transformed to make them more appropriate for parametric statistics (Cardinal \& Aitken, 2006). Violations can result in increased type 1 error, loss of power, and biased estimates, particularly with small sample sizes and unequal sample sizes (Glass, Peckham, \& Sanders,1972; Lix, Keselman, \& Keselman, 1996; Scheffe, 1959), as is the case with this data-set. Thus, non-parametric statistics were employed when transformations failed to uphold the assumption of normality, with critical ratios of skew above three and/or kurtosis above four (Kline, 2005). Transforming neuropsychological data when the data fail to meet parametric assumptions has been done by others in the field for CANTAB data (e.g., Dittrich et al., 2011; Morein-Zamir et al., 2014; Purcell et al., 1998b), including by the research group at Cambridge, who developed the CANTAB battery (e.g., Chamberlain et al., 2007). Transformations have also been used for other neuropsychological data, including the stop-signal task (e.g., Morein-Zamir, Fineberg, Robbins, \& Sahakian, 2010). The use of non-parametric 
statistics when statistical assumptions were violated has also routinely been used with neuropsychological data (e.g., Rao et al., 2008; Viswanath et al., 2009)

To assess group differences in demographic and clinical variables, chi-square tests were used for categorical variables (e.g., gender). One-way analysis of variance (ANOVA) was used for continuous variables (e.g., age, depressive symptoms). Differences on neuropsychological variables between groups were examined using ANOVAs with Tukey HSD post-hoc tests.

Importantly, this study used pre-existing groups (i.e., diagnostic status is not random group assignment) that in previous research have often differed from one another in demographic composition (e.g., Cohen et al., 1996; Bohne et al., 2005; Tolin et al., 2014) and in depressive symptoms (e.g., Morein-Zamir et al., 2014; Tolin et al., 2014). Consequently, the issue emerged of how to account for differences across groups in these variables. As has been highlighted in the literature (e.g., Miller \& Chapman, 2001), there is no agreed upon or theoretically sound method to statistically control for pre-existing group differences on variables related to group membership. Further complicating matters, two types of variables related to group membership have been proposed: variables conceptually connected to group membership, and background variables, not thought to have a conceptual relationship with group membership (van Eersel, Bouwmeester, Polak, \& Verkoeijen, 2017). Statistically controlling for background variables is arguably less problematic than controlling for variables conceptually related to group membership, which can result in uninterpretable results (van Eersel et al., 2017).

With these ideas in mind, in the current study, gender and age were considered to be background variables on which groups were expected to vary. For example, it is well established that women are over-represented in the GDs (e.g., Cohen et al., 1996; Gupta, Gupta, \& Knapp, 2015; Keuthen et al., 2010). It is also well established that a diagnosis of HD increases with age 
(e.g., Ayers, Saxena, Golshan, \& Wetherell, 2010; Grisham, Frost, Steketee, \& Hood, 2006; Kim, Steketee, \& Frost, 2001), with one study finding that the prevalence of HD increasing linearly by $20 \%$ with every 5 years of age (Cath, Nizar, Boomsma, \& Mathews, 2017). That noted, neither gender nor age are defining characteristics of the OCDRs. In contrast, in the current study, depressive symptoms were considered to be conceptually related to the group membership variable given the well-established relationship between depression and OCD (e.g., Pallanti, Grassi, Sarrecchia, Cantisani, \& Pellegrini, 2011). Indeed, the high rates of comorbidity between lifetime OCD and depression suggest that this comorbidity is not a coincidence (e.g., Kessler et al., 2005), and may indicate the presence of shared etiological factors between disorders. For example, one suggestion has been that negative affectivity may be an etiological factor shared between disorders (e.g., Watson, Clark, \& Greg, 1988; Stanton \& Watson, 2014). Thus, the shared variance between disorders may be conceptually significant to the construct, and any statistical removal of variance would be detrimental to the construct.

That noted, the norm, and often the recommendation in this literature, is to 'control' for depressive symptoms, particularly given that depressive symptoms have been reported to significantly influence neurocognitive task performance in OCD (Basso, Bornstein, Carona, \& Morton, 2001; Moritz et al., 2001). A regression approach for statistical control, rather than an Analysis of Covariance (ANCOVA), has been recommended (Miller \& Chapman, 2001) as an improvement over ANOVA (van Eersel et al., 2017). Although presenting with its own limitations, the regression approach to statistically managing real group differences includes what would have been a 'covariate' in the analysis as a predictor and substantive variable (Cohen \& Cohen, 1983; Harris, Bisbee, \& Evans, 1971). This approach still does not allow causal conclusions to be drawn (van Eersel et al., 2017). 
The current study's analytic plan attempted to balance what is done in this literature with the aforementioned statistical concerns associated with controlling for depression. Specifically, the current study adopted the following analytic approach: Standardized scores were used for neurocognitive variables, where available, to address group differences in gender and age. For variables that did not have standardized scores, group differences in the dependent variables were first examined without taking age, gender and depressive symptoms into account. ANOVA was used if parametric assumptions were met, and nonparametric techniques otherwise. The use of nonparametric statistics in a few instances did not regrettably allow consideration of control for other variables. When ANOVA was used and revealed a significant group difference in a dependent variable, the next analysis used a hierarchical regression that controlled in the first step for background variables (age and gender), then entered group membership in the second step (Field, 2013). If the group differences in the dependent variable persisted (such that group differences in the dependent variable emerged that were above and beyond group differences in demographic variables), a second hierarchical regression was conducted. ${ }^{3}$ In this second hierarchical regression, depressive symptoms, along with age and gender, were entered in the first step, followed by group membership. If group differences in the dependent variable disappeared when control for depressive symptoms was added, subsequent analysis was planned to demonstrate how the result could be explained as a function of the shared variance between the conceptually related variables of depressive symptoms and group membership. While the

\footnotetext{
${ }^{3}$ If group membership did not predict significant variance in the dependent variable after control for age and gender in the first hierarchical regression, it was deemed highly unlikely that significance for groups could occur in the second hierarchical regression including in step one age, gender, and depression. This is because including depression in step one likely would result in a "spurious underestimation" (Taylor et al., 2010, p. 167) of how well groups predicted the dependent variable. Nevertheless, for the interested reader, footnotes are included that denote the results of the second hierarchical regression with age, gender, and depression included when the first regression (with age and gender) did not reveal significance for groups.
} 
author acknowledges significant limitations with this approach (aforementioned) and conceptual implications of 'correcting' for variables that have a consequential relationship with the construct, this was conducted as a secondary analysis for the purpose of comparison with previous literature. It should be noted that another advantage of the regression approach over ANCOVA is that it also allows for the inclusion of interactions between predictors and group membership variables, enabling a test of whether the relationship between a dependent variable and a predictor varies across groups (Field, 2013). Unfortunately, the current study was unable to add interactions to the hierarchical regression, due to its small sample size relative to the number of predictors in the regression (Knofczynski \& Mundfrom, 2008). The results of these analyses should be interpreted in the context of the aforementioned limitations.

To examine the relationship between neurocognition, dysfunctional beliefs, and QOL life, Pearson correlations were computed between target variables and clinical groups (controls were excluded from the sample for this analysis). To examine how neurocognition predicted QOL, stepwise multiple regressions were utilized with the neurocognitive measures entered as predictor variables and scores on the QLES as the dependent variable. A probability of 0.05 was used to enter a variable in the equation, while a probability of 0.10 was used to remove a variable. The significance level was set at 0.01 . Effect size estimates are reported for all analysis. Most neurocognitive measures have multiple dependent variables, in some cases as many as twenty. Thus, as aforementioned, the inflated type I error rate in neuropsychological research has been highlighted in recent reviews as a significant limitation (Abramovitch et al., 2015; Kuelz et al., 2004). In particular, the need to correct for multiple comparisons in neuropsychological research has been emphasized in reviews of the neuropsychological literature in OCD (Abramovitch et al., 2015; Kuelz, Hohagen \& Vonderholzer, 2004). 
Thus, in addition to statistically controlling for multiple comparisons (described below), this study ran analyses only on the key dependent variables for each measure (e.g., the dependent variables most often reported in the literature for a given measure). To address for the issue of multiple comparisons (type 1 error), the Holm procedure (Holm, 1979) was used. This can be preferential to the traditional Bonferroni correction which, due to the numerous dependent variables in neuropsychological research, results in a significant reduction of power and an increase in type II error. The Holm procedure performs sequential Bonferroni corrections on each individual hypotheses, such that the adjusted $p$ value (alpha level) has to be smaller than .05 divided by the number of dependent variables for that measure. Thus, the tests are first performed and then ordered from the smallest $p$-value to the largest. Due to the significant aforementioned power limitations of this study, the Holm procedure was utilized over setting an overall conservative significance threshold of .01 , as the Holm procedure is 'less costly in terms of power' and is one method of addressing multiplicity adjustments that has been recommended in a systematic review of neuropsychological data in OCD (Abramovitch et al., 2015, p. 114). However, it should be noted that the use of the Holm procedure rather than the use of an overall conservative significance threshold does increase the chance of Type 1 error over the more conservative approach. The study-wide Type I error rate for the current study was between 1$.96^{4}$. Practically, this means the probability of Type 1 error, due to the vast number of comparisons, is between $96 \%-100 \%$. The results should be interpreted with this in mind.

\footnotetext{
${ }^{4}$ To calculate the study-wide error rate the following formula was used: FWE $\leq 1-\left(1-\alpha_{\mathrm{IT}}\right)^{\mathrm{c}}$ Where: $\alpha_{I T}=$ alpha level for an individual test and $\mathrm{c}=$ Number of comparisons. Due to the use of the Holm's procedure which performs sequential Bonferroni corrections, the alpha level varied from 0.5 to .0083 . As such, the error rate is given as a range.
} 


\section{Results}

\section{Demographic and Clinical Characteristics}

Table 1 displays the demographic characteristics of the four groups. The groups did not differ significantly on either IQ or education, $F(3,73)=2.57, p=.060 ; F(3,73)=1.21, p=.312$, respectively. However, age differed significantly between groups, $F(3,73)=9.17, p<.0001, \eta^{2}=$ .27. Specifically, individuals in the HD group were significantly older than all other groups (Tukey's HSD; $p<.05)$. There was no other significant age difference across groups. Of the GD group, ten individuals had a diagnosis of TTM (56\%) and eight individuals had a diagnosis of $\operatorname{ED}(44 \%)$.

Chi-square analysis revealed that gender also differed significantly across groups, $\chi^{2}(3)$ $=19.19, p<.0001, V=.499$. Post hoc comparisons revealed that relative to the control group (36.4\% female; $n=8)$, there was a significantly greater proportion of females than males in both the GD group $(94.4 \%$ female; $n=17)$ and the HD (87.5\% female; $n=14)$ group, $p<.05$. There were no other significant gender differences across groups.

Examination of comorbid psychological disorders in the clinical sample revealed that the majority of the clinical sample $(62 \% ; n=34)$ had at least one comorbid diagnosis (defined as a psychological disorder in addition to the primary diagnosis). The most frequently reported current comorbidities outside of the OCRDs included Major Depressive Disorder (28\%), Generalized Anxiety Disorder (7\%), Dysthymia (now called Persistent Depressive Disorder in DSM-V) (7\%), Anorexia Nervosa/Bulimia (5\%), Panic Disorder (7\%), Social Phobia (5\%), Posttraumatic Stress Disorder (2\%) and Specific Phobia (2\%). No psychological diagnoses were present in the control group. Chi-square analysis revealed that the number of comorbid conditions did not differ significantly across clinical groups, $\chi^{2}(2)=18.01, p=.70, V=.43$. 
Thirty-one percent of the clinical sample presented with at least one current additional OCRD. Specifically, in the OCD group, comorbid HD $(n=2)$ and GD $(n=5)$ were present. In the GD group, comorbid OCD $(n=3)$ and HD $(n=1)$ were present. Finally, in the HD group, comorbid $\mathrm{OCD}(n=3)$ and GD $(n=3)$ were present.

Based on participant self-report, a total of $67 \%$ of the clinical participants were taking psychopharmacological medication at the time of the study. Psychopharmacological treatment had remained stable for at least 6 weeks prior to study participation. Among participants receiving psychopharmacological treatment, $44 \%$ were on selective serotonin reuptake inhibitors (SSRIs), including sertraline, escitalopram, paroxetine, and fluoxetine. Of those participants on medication, 22\% were on serotonin norepinephrine reuptake inhibitors (SNRIs), including venlafaxine and duloxetine. An additional $8 \%$ of participants were prescribed benzodiazepines (e.g., clonazepam). Two clinical participants were prescribed clomipramine. One control participant was on the benzodiazepine, alprazolam, which was being prescribed as a muscle relaxant. 
Table 1

Sociodemographic characteristics of the sample

\begin{tabular}{|c|c|c|c|c|c|c|c|c|}
\hline & \multicolumn{2}{|c|}{ Control $(n=22)$} & \multicolumn{2}{|c|}{ OCD $(n=21)$} & \multicolumn{2}{|c|}{$\mathrm{GD}(n=18)$} & \multicolumn{2}{|c|}{$\mathrm{HD}(n=16)$} \\
\hline & $\mathrm{N}$ & $(\%)$ & $\mathrm{N}$ & $(\%)$ & $\mathrm{N}$ & $(\%)$ & $\mathrm{N}$ & $(\%)$ \\
\hline \multicolumn{9}{|l|}{ Gender } \\
\hline Male & 14 & 63.6 & 9 & 42.9 & 1 & 5.6 & 2 & 12.5 \\
\hline Female & 8 & 36.4 & 12 & 57.1 & 17 & 94.4 & 14 & 87.5 \\
\hline \multicolumn{9}{|l|}{ Ethnicity } \\
\hline Caucasian & 9 & 40.9 & 16 & 76.2 & 16 & 88.9 & 14 & 87.5 \\
\hline Asian & 9 & 40.9 & 2 & 9.5 & 1 & 5.6 & 1 & 6.3 \\
\hline A.A. & 2 & 9.1 & 0 & 0 & 1 & 5.6 & 0 & 0 \\
\hline H.L & 1 & 4.5 & 1 & 4.8 & 0 & 0 & 0 & 0 \\
\hline N.A. & 0 & 0 & 1 & 4.8 & 0 & 0 & 0 & 0 \\
\hline \multirow[t]{2}{*}{ Other } & 1 & 4.5 & 1 & 4.8 & 0 & 0 & 1 & 6.3 \\
\hline & M & SD & M & $\mathrm{SD}$ & M & SD & M & $\mathrm{SD}$ \\
\hline $\begin{array}{l}\text { Age } \\
\text { (years) }\end{array}$ & 33.50 & 15.34 & 36.90 & 12.08 & 35.28 & 11.05 & 53.06 & 8.68 \\
\hline $\begin{array}{l}\text { Education } \\
\text { (years } \\
\text { post- } \\
\text { secondary) }\end{array}$ & 2.89 & 1.96 & 4.02 & 2.28 & 3.78 & 1.99 & 3.13 & 2.57 \\
\hline $\begin{array}{l}\text { IQ } \\
\text { (WTAR) }\end{array}$ & 113.1 & 8.32 & 117.46 & 4.85 & 115.37 & 5.46 & 112.54 & 4.96 \\
\hline
\end{tabular}

Note. A.A. $=$ African American; H.L. = Hispanic/Latino; N.A. = Native American 


\section{Symptom Severity.}

As expected, there were significant differences between the control group and clinical groups on measures of symptom severity (Table 2), with those in the control group reporting significantly less symptoms of anxiety (as measured by the BAI) and depression (as measured by the BDI-II) than those in the OCD or HD groups (Tukey's HSD; $p<.05$ ), respectively. There was no significant difference between controls and those in the GD group on measures of either anxiety or depression. As a group, $\operatorname{OCD}(M=16.24, S D=8.72)$ and $\mathrm{HD}(M=18.65, S D=$ 10.81) participants reported mild symptoms of depression, while GD $(M=11.33, S D=8.72)$ participants and controls $(M=5.89, S D=5.97)$ reported minimal symptoms of depression. All three clinical groups displayed mild levels of overall anxiety, OCD $(M=15.40, S D=9.04)$; GD $(M=9.44, S D=9.54) ; \mathrm{HD}(M=13.08, S D=10.27)$, while controls $(M=4.32, S D=5.80)$ displayed minimal levels of anxiety, although the OCD group mean was on the cusp of moderate symptomatology. Of note, examination of anxiety and depressive symptoms between the clinical groups revealed no significant differences between groups (OCD, HD, GD) on general levels of

anxiety (measured by the BAI), $F(2,52)=2.76, p=.07, \eta^{2}=.096$, or depressive symptoms (measured by the BDI-II), $F(2,52)=1.89, p=.162, \eta^{2}=.068$.

While it can be counter-intuitive that the groups showed only minimal or mild anxiety/depressive symptoms, while $60 \%$ of the sample had a comorbid diagnosis (including Major Depressive Disorder and Generalized Anxiety Disorder), closer examination revealed a bimodal distribution of depressive/anxiety symptoms, such that those with a comorbid diagnosis had high levels of symptoms, and those without a comorbid diagnosis had no or minimal symptoms. 
Examination of OCD symptoms revealed that the OCD sample had moderate levels of OCD symptoms, as measured by the OCI-R and the Y-BOCS. The mean score on the OCI-R ( $M$ $=26.62, S D=9.67$ ) for the OCD group was comparable to the mean score often reported for individuals with OCD $(M=28, S D=13.53)$ (Foa et al., 2002), and well above the clinical cut-off for the measure of 21. Examination of hoarding symptoms revealed that the HD sample had moderate levels of HD symptoms, as measured by the SI-R. The mean score on the SI-R ( $M=$ 62.17, $S D=14.54)$ for the HD group was comparable to the mean score often reported for individuals with $\operatorname{HD}(M=62.0, S D=12.7)$ (Frost et al., 2004; Tolin, Meunier et al., 2011), and well above the clinical cut-off for the measure of 41. Examination of TTM and ED symptoms revealed that the GD sample when collapsed had mild levels of TTM symptoms as measured by the MGHPS $(M=8.45, S D=8.15)$ and mild levels ED symptoms $(M=9.00, S D=8.96)$ as measured by the SPS. This is considerably lower than the mean score often reported for individuals with TTM $(M=18 ; S D=5.1)$ (e.g., Weidt et al., 2015) or $\operatorname{ED}(M=12.74, S D=3.83)$ (Keuthen et al., 2001), presumably because some individuals with TTM did not have symptoms of ED and vice versa (thus could have 0 symptoms of the other disorder, which dramatically influences the mean). However, when the GD group was split into TTM and ED respectively, those with TTM had moderate levels of symptom severity $(M=15.5, S D=1.38)$ and those with ED had moderate levels of symptoms severity $(M=15.8, S D=4.60)$, comparable to the means of other published samples. ${ }^{5}$

\footnotetext{
${ }^{5}$ Global severity was also assessed using the global assessment of functioning (GAF) on the SCID-IV. There was missing data on this rating, as it was not an outcome of interest a priori; it was included after-the-fact for those whom this rating was available (missing data $n=34$ ). The GAF is a clinician rating of a patient's functioning. Scores range from 0 'severely impaired' to 100 'extremely high functioning'. GAF scores are given in Table 2.0. An ANOVA of the GAF data did not find that the clinical groups statistically differed from each other on GAF ratings, $F(2,18)=1.85, p=.185, \eta^{2}=.17$.
} 
Table 2

Clinical characteristics of the sample

\begin{tabular}{|c|c|c|c|c|c|c|c|c|}
\hline & \multicolumn{2}{|c|}{ Control $(n=22)$} & \multicolumn{2}{|c|}{$\underline{\mathrm{OCD}}(n=21)$} & \multicolumn{2}{|c|}{$\underline{\mathrm{GD}(n=18)}$} & \multicolumn{2}{|c|}{$\underline{\mathrm{HD}(n=16)}$} \\
\hline & M & SD & M & SD & M & SD & M & SD \\
\hline $\begin{array}{l}\text { Age of } \\
\text { onset } \\
\text { (years) }\end{array}$ & - & - & 16.35 & 11.94 & 12.31 & 8.69 & 26.00 & 17.50 \\
\hline $\begin{array}{l}\text { YBOCS } \\
\text { Total }\end{array}$ & - & - & 22.41 & 7.04 & - & - & - & - \\
\hline OCI-R & 7.27 & 7.68 & 26.62 & 9.67 & 10.61 & 8.57 & 22.53 & 11.72 \\
\hline Checking & 1.31 & 1.81 & 4.00 & 2.83 & 1.39 & 1.75 & 2.77 & 2.79 \\
\hline Hoarding & 2.00 & 2.79 & 2.67 & 2.37 & 2.61 & 3.22 & 8.54 & 2.79 \\
\hline Neut/Ob & 0.32 & 0.72 & 4.48 & 4.13 & 0.27 & 0.96 & 1.86 & 2.09 \\
\hline Ordering & 0.59 & 1.50 & 5.52 & 3.23 & 2.27 & 2.97 & 2.86 & 2.03 \\
\hline Washing & 2.36 & 2.61 & 5.29 & 3.72 & 2.77 & 3.86 & 4.29 & 2.50 \\
\hline OCI-OCD & 5.27 & 5.46 & 23.95 & 10.17 & 8.00 & 11.02 & 12.82 & 11.23 \\
\hline SI-R & 0.68 & 1.21 & 4.67 & 3.86 & 1.28 & 2.72 & 2.20 & 3.20 \\
\hline Clutter & 16.13 & 13.67 & 19.91 & 13.87 & 21.11 & 19.54 & 62.17 & 14.54 \\
\hline Discard & 5.22 & 5.87 & 6.57 & 6.70 & 8.05 & 9.31 & 26.17 & 5.72 \\
\hline Acquire & 4.91 & 4.77 & 8.09 & 5.34 & 6.39 & 6.69 & 19.26 & 4.36 \\
\hline & 8.18 & 3.86 & 7.52 & 2.85 & 8.78 & 3.79 & 16.86 & 4.70 \\
\hline MGHPS & - & - & - & - & 8.45 & 8.15 & - & - \\
\hline SP-SAS & - & - & - & - & 9.00 & 8.96 & - & - \\
\hline BDI-II & 5.89 & 5.97 & 16.24 & 8.72 & 11.33 & 8.72 & 18.68 & 10.81 \\
\hline BAI & 4.32 & 5.80 & 15.40 & 9.04 & 9.44 & 9.54 & 13.08 & 10.27 \\
\hline GAF & - & - & 58.70 & 8.54 & 65.80 & 3.83 & 57.67 & 8.26 \\
\hline
\end{tabular}

Note. $\mathrm{M}=$ Mean; $\mathrm{SD}=$ Standard deviation; $\mathrm{OCD}=$ obsessive-compulsive disorder; $\mathrm{GD}$ = grooming disorder; $\mathrm{HD}=$ hoarding disorder; YBOCS = Yale-Brown Obsessive-Compulsive Scale; OCI-R = Obsessive-Compulsive Inventory-Revised; Neut/Ob =

Neutralizing/Obsessing; OCI-OCD = OCI-R hoarding items removed; SI-R = Saving's Inventory - Revised; MGH = Massachusetts General Hospital Hair-Pulling Scale; SPS = Skin Picking Scale ; BDI-II = Beck Depression Inventory-II; BAI = Beck Anxiety Inventory 


\section{Neurocognitive Results}

See Table 3.0 for means and standard deviations of the neurocognitive measures.

\section{Inhibitory Dysfunction: Response inhibition.}

\section{Stop-Signal.}

For the analysis of the stop-signal data, 15 cases in total were excluded; of these, five cases had invalid trials based on the cut-off scores for the percentage correct and percentage inhibited (Schachar, 2009). An additional 10 cases had missing data due to issues with the software and licensing over the 2 years of data collection. Thus, the sample size for these analyses were as follows: control $(n=21), \mathrm{OCD}(n=17), \mathrm{GD}(n=13)$, and $\operatorname{HD}(n=10)$. For the SSRT data, all assumptions of parametric tests were met, such that the data distribution approximated normality and the variances did not differ significantly across groups. The a priori significance level was calculated using the Holm's procedure to account for multiple comparisons at 0.05 and .025 . ANOVA revealed a significant effect of group on SSRT, $F(3,57)$ $=7.39, p<.001, \eta_{\mathrm{p}}^{2}=.28$. Tukey post hoc analyses on the ANOVA data indicated that those in the HD group had significantly longer SSRT, indicating longer response inhibition, than did controls $(p=.001), \operatorname{OCD}(p=.002)$, and GD $(p=.001)$. No other significant group differences emerged.

A follow-up hierarchical regression that included the demographic variables (age and gender) in the model revealed that group membership remained a significant predictor of SSRT when age and gender were also included in the model, $\mathrm{r}^{2}$ change $=.18, F(2,58)=6.28, p=.003$. More specifically, in step 1 of this hierarchical regression, age and gender were entered, and age $(\mathrm{b}=.42, p=.001)$ but not gender $(\mathrm{b}=-.052, p=.663)$ predicted SSRT. When groups (coded as three columns denoting group membership) were added in the second step of the model, group remained a significant predictor of variance, $\mathrm{r}^{2}=.33, \mathrm{~F}(3,55)=64.14, p=.010$. More 
specifically, in step 2 of this hierarchical regression, groups were entered, and only the HD group was a significant predictor of variance in SSRT: for HD, $\mathrm{b}=.45, p=.003$; for OCD, $\mathrm{b}=.03, p=$ $.814 ;$ and for GD, $\mathrm{b}=-.015, p=.911$.

A second hierarchical regression that included both the demographic (age, gender) variables and the measure of depressive symptoms (BDI-II) again revealed that group remained a significant predictor of SSRT, $\mathrm{r}^{2}=.34, F(3,54)=3.55, p=.020$; in the second step of this model, $\mathrm{HD}(\mathrm{b}=.39, p=.015)$ but not the other variables were significant: gender $(\mathrm{b}=-.11, p=$ $.390)$, age $(\mathrm{b}=.23, p=.071)$, BDI-II $(\mathrm{b}=.136, p=.281)$, OCD $(\mathrm{b}=-.035, p=.806)$, and GD ( $\mathrm{b}$ $=-.056, p=.695)$.

The MRT data violated assumptions of normality (critical ratios of skew above three; Kline, 2005). Therefore, log transformation was used. No significant differences emerged between groups when examining MRT, $F(3,57)=.167, p=.919, \eta^{2} \mathrm{p}=.009{ }^{6}$

Inhibitory Dysfunction: Interference Control

Stroop Colour and Word Test

For the analyses of the Stroop data, no cases were excluded. The dependent variables had corresponding standardized T scores. Due to the significant group differences outlined above (e.g., age, gender), standardized $\mathrm{T}$ scores were utilized. The a priori significance level was calculated using the Holm's procedure to account for multiple comparisons at 0.05 and .025 . ANOVA revealed no significant differences between groups for either the colour-word score,

\footnotetext{
${ }^{6}$ Using the untransformed data, the analysis remained non-significant $F(3,57)=.66, p=.85, \eta_{\mathrm{p}}^{2}=.01$
} 
$F(3,73)=2.21, p=.094, \eta^{2}{ }_{p}=.083$, or the interference score, $F(3,73)=1.09, p=.357, \eta_{p}^{2}=$ .043 .

\section{Cognitive Flexibility: Set Shifting.}

\section{Wisconsin Card Sorting Task.}

For the analysis of the Wisconsin Card Sorting Task, one case had missing data and was excluded. Thus, the sample size for these analyses was as follows: control $(n=22), \operatorname{OCD}(n=$ $20)$, GD $(n=18)$, and HD $(n=16)$. Several of the dependent variables had corresponding standardized T scores. Due to the significant group differences outlined above (e.g., age, gender), standardized T scores were utilized when available. Six dependent variables were analyzed. The a priori significance level was calculated using the Holm's procedure to account for multiple comparisons at $.0083, .01, .013, .017, .025$, and .05 . The following dependent variables had available standardized scores; total errors, perseverative errors, and nonperseverative errors. ANOVA of the standardized scores revealed no significant group differences for total errors $F(3,72)=.595, p=.620, \eta_{\mathrm{p}}^{2}=.025$, perseverative errors $F(3,72)=.602, p=.616, \eta^{2} \mathrm{p}=.025$ or nonperseverative errors $F(3,72)=.808, p=.494, \eta_{\mathrm{p}}^{2}=.031$. The dependent variables, categories completed, trials administered, and total trials correct did not have standardized scores. All three variables violated the assumptions of normality, with critical ratios of skew above three; kurtosis above four (Kline, 2005). Data transformation did not resolve nonnormality; thus, nonparametric analyses were conducted. The Kruskal-Wallis test revealed number of trials administered $\left(H(3)=9.09, p=.025, \eta^{2}=.10\right)^{7}$ and total correct $(H(3)=7.54, p=$

\footnotetext{
${ }^{7}$ Using parametric analysis the results remained significant $F(3,72)=4.27, p=.008, \eta_{\mathrm{p}}^{2}=.15$; A follow-up hierarchical regression that included the demographic variables (age and gender) in the model More specifically, in step 1 of this hierarchical regression, age and gender were entered, and age $(b=.40, p<.001)$ but not gender $(b=-$ $.16, p=.14)$ predicted trials administered. In the second step, when groups were entered, group membership was no longer a significant predictor of WCST trials administered, $\mathrm{r}^{2}$ change $=.06, F(3,70)=1.71, p=.17$; age $(\mathrm{b}=.26, p$ $=.036)$, gender $(\mathrm{b}=-18, p=.144) \mathrm{HD}(\mathrm{b}=.27, p=.082), \mathrm{OCD}(\mathrm{b}=.04, p=.736), \mathrm{GD}(\mathrm{b}=-.05, p=.72$.
} 
$\left..05, \eta^{2}=.12\right)^{8}$ were significantly different between groups. Mann-Whitney tests were conducted as post hoc analyses. All effects are reported at a .0167 level of significance. Analyses revealed that the HD group had significantly more trials administered $(\mathrm{U}=97.50, p=.019, r=.38)$ and fewer total correct responses $(\mathrm{U}=92.0, p=.013, r=.40)$ than controls. No significant differences emerged between the OCD group $(\mathrm{U}=194.50, p=.518, r=.09 ; \mathrm{U}=200.0, p=.613$, $r=.08)$ and controls, or the GD group and controls $(\mathrm{U}=184.50, p=.712, r=.06 ; \mathrm{U}=190.50, p$ $=.838, r=.03$ ), on either trials administered or total correct responses, respectively. Those in the HD group had significantly more trials administered than the GD group $(\mathrm{U}=61.0, p=.004, r=$ $.49)$ and there was a trend towards more total correct $(\mathrm{U}=78.5, p=.023, r=.39)$. There was a trend towards those in the HD group having significantly more trials administered $(\mathrm{U}=98.50, p$ $=.049, r=.33)$ and fewer total correct responses $(\mathrm{U}=99.50, p=.053, r=.32)$ than the OCD group. There was no statistically significant difference between OCD and GD $(\mathrm{U}=148.50, p=$ $.356, r=.15 ; \mathrm{U}=163.0, p=.633, r=.08)$ on either trials administered or total correct responses, respectively. However, since nonparametric testing had to be employed, age, gender, and depressive symptoms could not be included in the model via regression to determine variance contributions. Categories completed correctly did not significantly differ between groups $H(3)=$ $4.97, p=.17, \eta^{2}=.07 .^{9}$

\section{Intradimensional/Extradimensional Task.}

For the analysis of the ID/ED data, there was one outlier that was removed. An additional 10 cases had missing data due to issues with the software and licensing over the 2 years of data

\footnotetext{
${ }^{8}$ Using parametric analysis the results were no longer significant $F(3,72)=1.54, p=.21, \eta^{2} \mathrm{p}=.06$. However, a case can be made that a parametric approach is inappropriate here. As such, the results from the nonparametric analysis will be emphasized instead.

${ }^{9}$ Using parametric statistics, the analysis remained nonsignificant $F(3,72)=1.87, p=.14, \eta^{2}=.07$.
} 
collection. Thus, the sample size for these analyses was as follows: control $(n=21)$, OCD $(n=$ 19), GD $(n=14)$, and HD ( $n=11)$. The a priori significance level was calculated using the Holm's procedure to account for multiple comparisons at 0.05 and .025 . Assumptions of parametric testing were violated for both dependent variables (with critical ratios of skew above three; kurtosis above four, Kline, 2005). A log transformation was used for total errors. Attempts to normalize the categories completed variable via transformation were unsuccessful; thus, nonparametric analyses were conducted. ANOVA revealed that total errors did not significantly differ between groups, $F(3,61)=2.09, p=.111, \eta^{2} \mathrm{p}=.093^{10}$. Kruskal-Wallis test revealed no significant difference between groups on stage completed, $H(3)=2.83, p=.425, \eta^{2}=.04 .{ }^{11}$

\section{Trail Making Test.}

For the analyses of the trail data, no cases were excluded. The a priori significance level was calculated using the Holm's procedure to account for multiple comparisons at 0.05 and .025 . Assumptions of parametric testing were violated for both dependent variables (with critical ratios of skew above three; Kline, 2005). A log transformation was used for both variables. ANOVA revealed a significant effect of group on number sequencing, $F(3,73)=5.15, p=0.003, \eta^{2}=$ $.175^{12}$ and letter number sequencing, $F(3,73)=3.03, p=0.035, \eta^{2}{ }_{p}=.111^{13}$. Tukey post hoc

\footnotetext{
${ }^{10}$ Using the untransformed data, the analysis remained nonsignificant $F(3,62)=2.23, p=.09, \eta_{\mathrm{p}}^{2}=.10$.

${ }^{11}$ Using the untransformed data, the analysis remained nonsignificant $F(3,62)=1.65, p=.19, \eta^{2} \mathrm{p}=.07$

${ }^{12}$ Using the untransformed data, the analysis remained significant $F(3,73)=4.64, p=.005, \eta_{\mathrm{p}}^{2}=.16$; A follow-up hierarchical regression that included the demographic variables (age and gender) in the model revealed that both gender and age were significant predictors of Trail A number sequencing, $\mathrm{r}^{2}$ change $=.17 ., F(2,74)=7.38, p=.001$; age $(\mathrm{b}=.30, p=.007)$ and gender $(\mathrm{b}=.24, p=.032)$. In the second step, when groups were entered, the model was no longer significant, $\mathrm{r}^{2}$ change $=.06 ., F(3,71)=1.87, p=.143$. Despite the fact that the overall model was not significant, the OCD group did, in fact, significantly predict variance; age $(\mathrm{b}=.23, p=.061)$, gender $(\mathrm{b}=.19, p=$ $.128), \mathrm{HD}(\mathrm{b}=.23, p=.133)$, OCD $(\mathrm{b}=.29, p=.026), \mathrm{GD}(\mathrm{b}=.13, p=.365)$. A second hierarchical regression that included both demographic variables (age \& gender) and the measure of depressive symptoms (BDI-II) in the first step and group in the second step revealed that depressive symptoms did not contribute significant variance to the model $(\mathrm{b}=.07, p=.576)$.

${ }^{13}$ Using the untransformed data, the analysis remained significant $F(3,73)=3.33, p=.024, \eta_{\mathrm{p}}^{2}=.12$; A follow-up hierarchical regression that included the demographic variables (age and gender) in the model revealed that age was
} 
analyses on the number sequencing ANOVA data indicated that those in the OCD group and HD group had significantly higher scores on number sequencing compared to controls (indicating that it took them longer to complete the task); $p=.031$, and $p=.002$, respectively. No other significant group differences emerged. Tukey post hoc analyses on the letter number sequencing ANOVA data indicated that those in the HD group had significantly higher scores on letter number sequencing compared to control (indicating that it took them longer to complete the task); $p=.025$. No other significant group differences emerged.

A follow-up hierarchical regression on the number sequencing data that included the demographic variables (age and gender) in the model revealed age and gender were significant predictors; $\mathrm{r}^{2}$ change $=.19, F(2,74)=8.81, p<.001 ;$ age $(\mathrm{b}=.33, p=.003)$ and gender $(\mathrm{b}=.25$, $p=.022$ ). In step 2 when groups were added to the model, the model was no longer significant, $\mathrm{r}^{2}$ change $=.61, F(3,71)=1.94, p=.13$. Despite the fact that the overall model was not significant, the OCD group did, in fact, significantly predict variance; age $(b=.26, p=.033)$, gender $(\mathrm{b}=.19, p=.110), \mathrm{HD}(\mathrm{b}=.23, p=.119), \mathrm{OCD}(\mathrm{b}=.29, p=.022), \mathrm{GD}(\mathrm{b}=.14, p=$ $.296) .{ }^{14}$

A follow-up hierarchical regression on the letter-number sequencing data that included the demographic variables (age and gender) in the first step of the model and group in the second step of the model revealed that age $(\mathrm{b}=.46, p=<.001)$ but not gender $(\mathrm{b}=-.03, p=.76)$

a significant predictor of Trail B letter number sequencing; $\mathrm{r}^{2}$ change $=.20 ., F(2,74)=9.13, p<.001 ;$ age $(\mathrm{b}=.45$, $p<.001)$ and gender $(\mathrm{b}=-.14, p=.910)$. $)$. In the second step, when groups were entered, the model was no longer significant, $\mathrm{r}^{2}$ change $=.02 ., F(3,71)=.73, p=.538$; age $(\mathrm{b}=.37, p=.003)$, gender $(\mathrm{b}=-.05, p=.666), \mathrm{HD}(\mathrm{b}=$ $.20, p=.189), \mathrm{OCD}(\mathrm{b}=.14, p=.286), \mathrm{GD}(\mathrm{b}=.07, p=.629)$.

${ }^{14} \mathrm{~A}$ second hierarchical regression that included both the demographic (age, gender) variables and the measure of depressive symptoms (BDI-II) in the first step and groups in the second step again revealed that group was not a significant predictor of number sequencing, $\mathrm{r}^{2}=.23, \mathrm{~F}(3,70)=1.12, p=.349$. Depressive symptoms did not contribute to significant variance in the model $(\mathrm{b}=.179, p=.093)$. 
predicted letter-numbering sequencing; $\mathrm{r}^{2}$ change $=.21, F(2,74)=9.83, p<.001$. When groups were added in the second step of the model, group did not remain a significant predictor of variance, $\mathrm{r}^{2}$ change $=.23, F(3,71)=.62, p=.60 ;$ age $(\mathrm{b}=.40, p=.002)$, gender $(\mathrm{b}=-.08, p=$ $.516), \mathrm{HD}(\mathrm{b}=.18, p=.222), \mathrm{OCD}(\mathrm{b}=.14, p=.282), \mathrm{GD}(\mathrm{b}=.09, p=.510) .{ }^{15}$

\section{Memory}

\section{Nonverbal Memory.}

\section{Rey Complex Figure Task.}

For the analyses of the RCFT, 2 cases were excluded due to incomplete data. Thus, the sample size for these analyses was as follows: control $(n=22), \mathrm{OCD}(n=21), \mathrm{GD}(n=17)$, and $\mathrm{HD}(n=15)$. The a priori significance level was calculated using the Holm's procedure to account for multiple comparisons at $0.05, .025$, and .016 . Assumptions of parametric testing were violated for the copy dependent variable (data violated assumption of normality, with critical ratios of skew above three; Kline, 2005). Attempts to transform the copy variable were unsuccessful. Thus, a nonparametric analysis was conducted. The Kruskal-Wallis test revealed a significant difference between groups, $H(3)=26.09, p<.001, \eta^{2}=.35 .{ }^{16}$ Mann-Whitney tests were conducted as post hoc analyses. All effects are reported at a .0167 level of significance. The

\footnotetext{
${ }^{15}$ A second hierarchical regression that included both the demographic (age, gender) variables and the measure of depressive symptoms (BDI-II) again revealed that group no longer remained a significant predictor of number sequencing, $\mathrm{r} 2=.27, \mathrm{~F}(3,70)=.033, p=.992$. Depressive symptoms contributed to significant variance in the model $(\mathrm{b}=.25, p=.017)$.

${ }^{16}$ Using parametric statistics, the analysis remained significant $F(3,72)=14.67, p<.001, \eta^{2} \mathrm{p}=.38$. A follow-up hierarchical regression that included the demographic variables (age and gender) in the model revealed that gender was a significant predictor; $\mathrm{r}^{2}$ change $=.17, F(2,73)=7.57, p=.001$; age $(\mathrm{b}=-.17, p=.116)$ gender $(\mathrm{b}=-.35, p=$ $.002)$. In the second step, when groups were entered, the model remained significant; $\mathrm{r}^{2}$ change $=.22, F(3,70)=$ $8.32, p<.001$; age $(\mathrm{b}=-.05, p=.65)$, gender $(\mathrm{b}=-.10, p=.34), \mathrm{HD}(\mathrm{b}=-.46, p=.001), \mathrm{OCD}(\mathrm{b}=-.10, p=.410)$, $\mathrm{GD}(\mathrm{b}=-.53, p<.001)$.
} 
copy variable was significantly different between control and GD $(\mathrm{U}=36.50, p<.001, r=.56)$ and HD $(\mathrm{U}=60.00, p=.001, r=0.68)$ but not between controls and OCD $(\mathrm{U}=161.00, p=.084$, $r=.26$ ), with those in the HD and GD group having a significantly lower copy scores than controls. Those in the OCD group had significantly higher copy scores than those in the GD group $(\mathrm{U}=63.00, p=.001, r=.55)$ and the HD group $(\mathrm{U}=84.50, p=.009, r=.42)$ respectively. There was no significant difference between the GD and HD groups on the copy variable $(\mathrm{U}=132.50, p=.90, r=.02)$. However, since nonparametric testing had to be employed, age, gender, and depressive symptoms could not be included in the model via regression to determine variance contributions.

For the immediate and delayed recall trials, both variables met parametric assumptions, such that the data distribution approximated normality and the variances did not differ significantly across groups. ANOVA revealed a significant effect of group on immediate recall, $F(3,71)=4.78, p<.004, \eta^{2}=.17$ and delayed recall, $F(3,69)=4.59, p<.01, \eta^{2}=.17$. Tukey post hoc analyses on the immediate recall ANOVA data indicated that those in the GD and HD groups recalled significantly less than controls $(p=.012, \mathrm{p}=.009)$. No other significant group differences emerged. Tukey post hoc analyses on the delayed recall ANOVA data indicated that those in the HD group recalled significantly less than controls $(p=.003)$. No other significant group differences emerged.

For the immediate recall data, a follow-up hierarchical regression that included the demographic variables (age and gender) in the model revealed that group no longer significantly predicted immediate recall when age and gender were also included in the model, $\mathrm{r}^{2}=.27, F(3$, 69) $=1.91, p=.135$. More specifically, in step 1 of this hierarchical regression, age and gender 
were entered, and age $(\mathrm{b}=-.37, p=.001)$ but not gender $(\mathrm{b}=-.20, p=.067)$ predicted immediate recall. ${ }^{17}$

For the delayed recall data, a follow-up hierarchical regression that included the demographic variables (age and gender) in the model revealed that group no longer significantly predicted immediate recall when age and gender were also included in the model, $\mathrm{r}^{2}=.28, F(3$, $67)=1.95, p=.130$. More specifically, in step 1 of this hierarchical regression, age and gender were entered, and age $(\mathrm{b}=-.45, p<.001)$ but not gender $(\mathrm{b}=-.056, p=.601)$ predicted delayed recall. $^{18}$

\section{Verbal Memory.}

\section{California Verbal Learning Test.}

For the analyses of the CVLT data, no cases were excluded. The a priori significance level was calculated using the Holm's procedure to account for multiple comparisons at 0.05 , $.025, .016 ; .013$; and .01 . The dependent variable, the sum of words recalled for trials $1-5$, met parametric assumptions, such that the data distribution approximated normality and the variances did not differ significantly across groups. ANOVA revealed that group did not significantly relate to total words recalled, $F(3,73)=.264, p=.851, \eta^{2}{ }_{\mathrm{p}}=.011$. The remaining variables violated parametric assumptions (data violated assumption of normality, with critical ratios of skew above three; Kline, 2005) and square root transformations were used successfully to

\footnotetext{
${ }^{17}$ A second hierarchical regression that included both the demographic (age, gender) variables and the measure of depressive symptoms (BDI-II) again revealed that group no longer remained a significant predictor of immediate recall, $\mathrm{r}^{2}=.27, F(3,68)=1.70, p=.18$. Depressive symptoms did not contribute significantly to the variance of the model $(\mathrm{b}=-.10, p=.361)$.

${ }^{18}$ A second hierarchical regression that included both the demographic (age, gender) variables and the measure of depressive symptoms (BDI-II) again revealed that group no longer remained a significant predictor of delayed recall, $\mathrm{r}^{2}=.28, \mathrm{~F}(3,66)=1.45, p=.20$. Depressive symptoms did not contribute significantly to the variance of the model $(\mathrm{b}=-.14, p=.198)$.
} 
normalize. ANOVA revealed a nonsignificant finding for group on short delay free recall, $F(3,72)=.858, p=.468, \eta^{2}{ }_{p}=.052^{19} ;$ long delay free recall $F(3,72)=.69, p=.559, \eta^{2}{ }_{p}=$ $.028^{20}$; total number of intrusions $F(3,72)=.729, p=.538, \eta^{2} \mathrm{p}=.029^{21}$; and total number of repetition $F(3,72)=.658, p=.581, \eta^{2} \mathrm{p}=.027^{22}$. The dependent variable semantic clustering index also violated parametric assumptions (data violated assumption of normality, with critical ratios of skew above three; Kline, 2005) and a square root transformation was used successfully to normalize data. The data violated the homogeneity of variance test, $F(3,73)=13.20, p<.001$. Therefore, Welch's F was reported. The ANOVA revealed that group was not significantly associated with semantic clustering, Welch's $F(3,39.68)=0.71, p=.55, \eta^{2} \mathrm{p}=.034 .^{23}$

\section{Processing Speed.}

\section{Digit Symbol.}

For the analysis of the digit symbol, there was no missing data and data met parametric assumptions, such that the data distribution approximated normality and the variances did not differ significantly across groups. ANOVA revealed no significant effect of group on digit symbol, $F(3,73)=2.00, p=0.122, \eta^{2}=.076$.

\section{Working Memory.}

\section{Spatial Working Memory Task.}

For the analysis of the SWM data, 10 cases had missing data due to issues with the software and licensing. Thus, the sample size for these analyses was as follows: control $(n=21)$, OCD $(n=19), \operatorname{GD}(n=14)$, and HD $(n=11)$. The a priori significance level was calculated

\footnotetext{
${ }^{19}$ Using the untransformed data, the analysis remained nonsignificant $F(3,72)=.46, p=.717, \eta_{\mathrm{p}}^{2}=.02$.

${ }^{20}$ Using the untransformed data, the analysis remained nonsignificant $F(3,72)=.16, p=.92, \eta^{2}=.01$.

${ }^{21}$ Using the untransformed data, the analysis remained nonsignificant $F(3,72)=.73, p=.54, \eta^{2}=.03$.

${ }^{22}$ Using the untransformed data, the analysis remained nonsignificant $F(3,72)=.11, p=.37, \eta_{\mathrm{p}}^{2}=.04$.

${ }^{23}$ Using the untransformed data, the analysis remained nonsignificant $F(3,72)=2.51, p=.07, \eta^{2}=.14$.
} 
using the Holm's procedure to account for multiple comparisons at $0.05, .025$. The between search errors variable violated parametric assumptions. A square root transformation was used. ANOVA revealed a nonsignificant association between group and between search errors, $F(3,62)$ $=2.53, p=.065, \eta_{\mathrm{p}}^{2}=.109^{24}$. Attempts to transform the strategy variable were unsuccessful; thus, nonparametric analyses were conducted. Kruskal-Wallis test revealed that groups did not significantly differ on strategy.

\footnotetext{
${ }^{24}$ Using the untransformed data, the analysis became significant $F(3,62)=2.71, p=.05, \eta_{\mathrm{p}}^{2}=.12$. A follow-up hierarchical regression that included the demographic variables (age and gender) in the model revealed that age was a significant predictor; $\mathrm{r}^{2}$ change $=.22, F(2,63)=9.06, p<.001 ;$ age $(\mathrm{b}=.48, p<.001)$ gender $(\mathrm{b}=-.09, p=.417)$. In the second step, when groups were entered, the model was no longer significant; $\mathrm{r}^{2}$ change $=.02, F(3,60)=.613$, $p=.609 ;$ age $(\mathrm{b}=.43, p=.002)$, gender $(\mathrm{b}=-.07, p=.572), \mathrm{HD}(\mathrm{b}=.04, p=.791), \mathrm{OCD}(\mathrm{b}=-.12, p=.349)$, GD $(\mathrm{b}=-.11, p=.428)$.
} 
Table 3

Performance on neurocognitive measures of patients with obsessive-compulsive disorder (OCD), hoarding disorder (HD), a grooming disorder (GD), and healthy controls (HC)

\begin{tabular}{|c|c|c|c|c|c|c|c|c|}
\hline & \multicolumn{2}{|c|}{$\underline{\text { Control }}$} & \multicolumn{2}{|c|}{$\underline{\mathrm{OCD}}$} & \multicolumn{2}{|c|}{$\underline{\mathrm{GD}}$} & \multicolumn{2}{|c|}{$\underline{\mathrm{HD}}$} \\
\hline & M & (SD) & M & (SD) & M & (SD) & M & (SD) \\
\hline \multicolumn{9}{|l|}{ Stop-Signal } \\
\hline SSRT & 293.96 & 81.72 & 298.61 & 86.05 & 277.88 & 86.02 & 437.04 & 117.17 \\
\hline MRT & 738.58 & 193.48 & 767.58 & 207.27 & 760.50 & 231.96 & 816.09 & 319.25 \\
\hline \multicolumn{9}{|l|}{ Stroop } \\
\hline colour-word score & 49.50 & 10.86 & 50.38 & 9.29 & 54.94 & 11.63 & 46.06 & 8.51 \\
\hline interference & 51.81 & 10.24 & 52.93 & 8.66 & 55.78 & 7.83 & 50.65 & 7.82 \\
\hline \multicolumn{9}{|l|}{ WCST } \\
\hline total errors, & 50.77 & 11.60 & 48.20 & 11.07 & 52.11 & 5.35 & 47.81 & 15.68 \\
\hline perseverative error & 53.14 & 15.38 & 48.60 & 11.75 & 50.94 & 7.37 & 48.63 & 13.79 \\
\hline nonperseverative error & 51.27 & 8.63 & 49.75 & 9.98 & 53.39 & 6.66 & 48.25 & 14.73 \\
\hline categories completed & 5.36 & 1.71 & 5.45 & 1.50 & 6.00 & 0 & 4.81 & 1.83 \\
\hline trials administered & 86.68 & 21.14 & 88.55 & 21.09 & 80.28 & 11.06 & 103.81 & 23.37 \\
\hline total trials correct & 66.32 & 7.86 & 67.20 & 11.25 & 67.61 & 7.27 & 73.00 & 13.51 \\
\hline \multicolumn{9}{|l|}{ TMT } \\
\hline Number sequencing & 21.63 & 6.19 & 29.74 & 11.31 & 27.35 & 9.99 & 32.80 & 10.37 \\
\hline Letter/number & 50.59 & 19.15 & 60.70 & 27.55 & 54.40 & 20.26 & 76.26 & 36.00 \\
\hline \multicolumn{9}{|l|}{ ID/ED } \\
\hline stages completed & 8.33 & .966 & 8.26 & .933 & 8.57 & .851 & 7.27 & 2.41 \\
\hline total errors & 28.67 & 27.45 & 33.12 & 22.39 & 23.36 & 20.41 & 60.82 & 58.05 \\
\hline
\end{tabular}




\begin{tabular}{|c|c|c|c|c|c|c|c|c|}
\hline & \multicolumn{2}{|c|}{ Control } & \multicolumn{2}{|c|}{$\underline{\mathrm{OCD}}$} & \multicolumn{2}{|c|}{$\underline{\mathrm{GD}}$} & \multicolumn{2}{|c|}{$\underline{\mathrm{HD}}$} \\
\hline & M & (SD) & M & (SD) & M & (SD) & M & (SD) \\
\hline \multicolumn{9}{|l|}{ RCFT } \\
\hline Copy & 33.18 & 2.61 & 31.50 & 4.11 & 24.59 & 5.91 & 25.13 & 7.02 \\
\hline Immediate & 21.84 & 6.87 & 19.38 & 5.00 & 16.18 & 5.77 & 15.50 & 5.29 \\
\hline Delayed & 20.93 & 6.65 & 17.95 & 5.96 & 16.49 & 5.48 & 13.61 & 5.27 \\
\hline \multicolumn{9}{|l|}{ CVLT } \\
\hline trial 1-5 & 59.73 & 15.33 & 59.76 & 12.59 & 55.89 & 12.36 & 58.33 & 9.39 \\
\hline short delay recall & 12.77 & 3.32 & 11.67 & 3.59 & 11.67 & 3.22 & 12.46 & 2.36 \\
\hline long delay recall & 12.50 & 3.46 & 12.86 & 2.71 & 12.28 & 2.84 & 12.33 & 1.98 \\
\hline free recall intrusion & 2.77 & 3.26 & 2.57 & 3.26 & 2.22 & 3.57 & 3.00 & 4.12 \\
\hline total repetitions & 5.45 & 4.61 & 6.33 & 6.50 & 4.39 & 3.26 & 7.47 & 5.91 \\
\hline semantic index & 3.20 & 3.19 & 2.11 & 2.06 & 1.73 & 1.23 & 1.86 & 1.07 \\
\hline \multicolumn{9}{|l|}{ SWM } \\
\hline Between search errors & 29.59 & 24.91 & 25.05 & 21.94 & 21.21 & 18.19 & 45.36 & 23.01 \\
\hline Strategy score & 34.05 & 6.00 & 31.79 & 6.15 & 31.79 & 6.00 & 36.73 & 6.00 \\
\hline \multicolumn{9}{|l|}{ Digit Symbol } \\
\hline Number completed & 80.73 & 20.19 & 72.95 & 17.12 & 79.00 & 15.36 & 67.31 & 20.46 \\
\hline
\end{tabular}

Note. $\mathrm{M}=$ Mean; $\mathrm{SD}=$ Standard deviation; $\mathrm{OCD}=$ obsessive-compulsive disorder; $\mathrm{GD}=$ grooming disorder; $\mathrm{HD}=$ hoarding disorder; $\mathrm{SSRT}=$ stop signal reaction time; $\mathrm{MRT}=$ mean reaction time; $\mathrm{WCST}=\mathrm{Wisconsin}$ Card Sorting Task; TMT $=$ Trail Making Test; ID/ED = Intradimensional/Extradimensional Task; REY $=$ Rey Complex Figure Task; CVLT $=$ California Verbal Learning Test; SWM = Spatial Working Memory Task 


\section{Dysfunctional Beliefs}

See table 4.0 for means and standard deviations of dysfunctional belief measures.

\section{Obsessional Beliefs.}

For the analyses of the self-report belief domains, as measured by the OBQ, no cases were excluded. Data met the assumptions of normality, such that the data distribution approximated normality and the variances did not differ significantly across groups. The a priori significance level was calculated using the Holm's procedure to account for multiple comparisons at $0.05, .025, .016 ; .013$; and .01 . ANOVA revealed a significant association between group and OBQ scores, $F(3,73),=6.46, p=.001, \eta^{2} \mathrm{p}=.21$. Tukey post hoc analyses on the ANOVA data indicated that those in the OCD group had significantly inflated beliefs compared to controls $(p=.003)$, GD $(p=.007)$, and HD $(p=.003)$. Those in the GD and HD groups did not significantly differ from controls.

A follow-up hierarchical regression that included the demographic variables (age and gender) in the model revealed that group remained a significant predictor of self-report OBQ belief domains when age and gender were included in the model, $\mathrm{r}^{2}=.26$., $F(3,71)=6.15, p=$ .001 . More specifically, in step 1 of this hierarchical regression, age and gender were entered, and age $(b=-.23, p=.044)$, but not gender $(b=-.07, p=.516)$ contributed significant variance to the model. In step 2 of this hierarchical regression, groups were entered, and the OCD group $(\mathrm{b}=.48, p<.001)$, but not $\mathrm{HD}(\mathrm{b}=.12, p<.408)$ or $\mathrm{GD}(\mathrm{b}=.05, p=.716)$ contributed significant variance to the model.

A second hierarchical regression that included both the demographic (age, gender) variables and the measure of depressive symptoms (BDI-II) revealed that group remained a significant predictor of total OBQ belief domains, $\mathrm{r}^{2}$ change $=.36, F(3,70)=4.14, p=.009$; in 
the second step of this model, BDI-II $(b=.38, p=.001)$ and OCD $(b=.30, p=.023)$ but not GD $(\mathrm{b}=-.04, p=.746), \mathrm{HD}(\mathrm{b}=-.10, p=.520)$, gender $(\mathrm{b}=-.60, p=.590)$, or age $(\mathrm{b}=-.22, p=$ .057) contributed significant variance to the model.

Responsibility/Threat. The ANOVA revealed that group was significantly associated with responsibility/threat beliefs, $F(3,73)=6.73, p<.001, \eta_{\mathrm{p}}^{2}=.22$. Tukey post hoc analyses on the significant ANOVA data indicated that those in the OCD group reported significantly more responsibility/threat than controls $(p=.009), \mathrm{GD}(p=.011)$ and $\mathrm{HD}(p=.005)$.

A follow-up hierarchical regression that included the demographic variables (age and gender) in the model revealed that neither age nor gender predicted significant variance $r^{2}$ change $=.06, F(2,74)=2.33, p=.104 ;$ age $(b=-.22, p=.054)$, gender $(b=-.07, p=.549)$. In step 2 of this hierarchical regression, groups were entered and predicted significant variance in the model, $\mathrm{r}^{2}$ change $=.21, F(3,71)=6.79, p<.001 ;$ age $(\mathrm{b}=-.23, p=.058)$, gender $(\mathrm{b}=-.05, p=.678)$ and the OCD group $(\mathrm{b}=.50, p<.001)$, but not HD $(\mathrm{b}=.10, p=.477)$ or GD $(\mathrm{b}=.05, p=.720)$ contributed significant variance to the model.

A second hierarchical regression that included both the demographic (age, gender) variables and the measure of depressive symptoms (BDI-II) again revealed that group remained a significant predictor, $\mathrm{r}^{2}$ change $=.14, F(3,70)=4.82, p=.004$; in the second step of this model, OCD $(b=.34, p=.011)$ and BDI-II $(b=.33, p=.005)$ significantly predicted variance; gender $(\mathrm{b}=-.05, p=.661)$, age $(\mathrm{b}=-.20, p=.079), \mathrm{GD}(\mathrm{b}=-.03, p=.812), \mathrm{HD}(\mathrm{b}=-.08, p=.568)$.

Perfectionism/ Uncertainty. The ANOVA revealed that group was significantly associated with perfectionism/uncertainty, $F(3,73)=4.26, p<.01, \eta^{2}{ }_{p}=.15$. Tukey post hoc analyses on the significant ANOVA data indicated that those in the OCD group reported significantly more perfectionism/uncertainty than controls $(p=.007)$. 
A follow-up hierarchical regression that included the demographic variables (age and gender) in the model revealed that neither age nor gender predicted significant variance $\mathrm{r}^{2}$ change $=.04, F(2,74)=1.58, p=.213$; age $(b=-.19, p=.094)$, gender $(b=-.03, p=.787)$. In step 2 of this hierarchical regression, groups were entered and predicted significant variance in the model, $\mathrm{r}^{2}$ change $=.15, F(3,71)=4.43, p=.007 ;$ age $(\mathrm{b}=-.24, p=.057)$, gender $(\mathrm{b}=-.09, p=.437)$ and the OCD group $(\mathrm{b}=.47, p=.001)$, but not HD $(\mathrm{b}=.25, p=.106)$ or GD $(\mathrm{b}=.21, p=.135)$ contributed significant variance to the model.

A second hierarchical regression that included both the demographic (age, gender) variables and the measure of depressive symptoms (BDI-II) revealed that group no longer remained a significant predictor, $\mathrm{r}^{2}$ change $=.70, F(3,70)=2.23, p=.093$. Nevertheless, in the second step of this model, OCD $(\mathrm{b}=.32, p=.022)$ and BDI-II $(\mathrm{b}=.31, p=.013)$ significantly predicted variance, despite overall group not contributing significant variance; gender $(b=-.09$, $p=.418)$, age $(\mathrm{b}=-.217, p=.077), \mathrm{GD}(\mathrm{b}=.14, p=.320), \mathrm{HD}(\mathrm{b}=.07, p=.661)$.

Importance/Control of Thoughts. The data violated the homogeneity of variance test $F(3,73)=4.39, p=.007$. Therefore, Welch's $\mathrm{F}$ was reported. The ANOVA revealed that group was significantly associated with importance/control of thoughts, Welch's $F(3,40.37)=3.63, p$ $=.021, \eta_{\mathrm{p}}^{2}=.14$. Games-Howell was employed as a post-hoc test, which revealed that those in the OCD group reported significantly more importance/control of thoughts than those in the GD group $(p=.032)$ and those in the HD group $(p=.039)$.

A follow-up hierarchical regression that included the demographic variables (age and gender) in the model revealed that neither age nor gender predicted significant variance $\mathrm{r}^{2}$ change $=.05, F(2,74)=2.02, p=.139 ;$ age $(b=-.19, p=.106)$, gender $(b=-.10, p=.367)$. In step 2 of this hierarchical regression, groups were entered and predicted significant variance in the model, 
$\mathrm{r}^{2}$ change $=.13, F(3,71)=3.64, p=.017 ;$ age $(\mathrm{b}=-.17, p=.189)$, gender $(\mathrm{b}=.00, p=.976)$,

$\operatorname{OCD}(\mathrm{b}=.25, p=.065), \mathrm{HD}(\mathrm{b}=-.08 ., p=.598), \mathrm{GD}(\mathrm{b}=-.19, p=.185)$.

A second hierarchical regression that included both the demographic (age, gender) variables and the measure of depressive symptoms (BDI-II) again revealed group remained a significant predictor, $\mathrm{r}^{2}$ change $=.11, F(3,70)=3.58, p=.018$; gender $(\mathrm{b}=.00, p=.977)$, age $(\mathrm{b}$ $=-.14, p=.255), \mathrm{GD}(\mathrm{b}=-.28, p=.047), \mathrm{HD}(\mathrm{b}=-.29, p=.073), \mathrm{OCD}(\mathrm{b}=.07, p=.611), \mathrm{BDI}-$ II $(\mathrm{b}=.36, p=.003)$.

\section{Intolerance of Uncertainty.}

For the analyses of the IU data, no cases were excluded. The a priori significance level was calculated using the Holm's procedure to account for multiple comparisons at 0.05 . The data violated the homogeneity of variance test, $F(3,73)=3.07, p=.033$. Therefore, Welch's F was reported. The ANOVA revealed that group was significantly associated with IU, Welch's $F(3$, $38.93)=6.46, p=.001, \eta_{p}^{2}=.18$. Games-Howell was employed as a post-hoc test, which revealed that those in the OCD group reported significantly more IU than controls $(p=.040)$ or those in the GD group $(p=.012)$. Those in the HD group reported significantly more IU than those in the GD group $(p=.049)$.

A follow-up hierarchical regression that included the demographic variables (age and gender) in the model revealed that group remained a significant predictor of self-report IU when age and gender were included in the model, $\mathrm{r}^{2}$ change $=.24, F(3,71)=7.23, p<.001$. More specifically, in step 1 of this hierarchical regression, age and gender were entered, neither age (b $=-.10, p=.396)$ nor gender $(b=.019, p=.871)$ contributed significant variance to the model. In step 2 of this hierarchical regression, groups were entered, and the OCD group $(b=.40, p<$ 
$.002), \mathrm{HD}(\mathrm{b}=.46, p=.003)$, and age $(\mathrm{b}=.30, p=.014)$ but not $\mathrm{GD}(\mathrm{b}=-.04, p=.788)$ or gender $(b=.01, p=.972)$ contributed significant variance to the model.

A second hierarchical regression that included both the demographic (age, gender) variables and the measure of depressive symptoms (BDI-II) revealed that while the overall model remained significant, group no longer accounted for significant variance when depressive symptoms were included in the model. $\mathrm{r}^{2}$ change $=.39, F(3,70)=3.07, p=.034$; BDI-II $(\mathrm{b}=$ $.45, p<.001) \mathrm{OCD}(\mathrm{b}=.18, p=.15) ; \mathrm{GD}(\mathrm{b}=-.15, p=.25), \mathrm{HD}(\mathrm{b}=.20, p=.191)$, gender $(\mathrm{b}=$ $.004, p=.972)$, or age $(b=-.27, p=.018)$.

Because group was no longer significant when depressive symptoms were entered in the previous analysis, a third hierarchical regression was conducted that reversed the order of entry of depressive symptoms and groups. Specifically, in step 1, age and gender were added to the model. In step 2, groups were added to the model. Groups were significant; specifically, the 2 change was .23 and the OCD group $(b=.40, p=.002), \mathrm{HD}(\mathrm{b}=.46, p=.003)$ were significant, as before. In step 3, depressive symptoms were added to the model. This was done to demonstrate whether this finding (that groups was no longer significant in the above analysis when depression was also included) is a result of shared variance (e.g., whether significance depends on which variable is entered into the model first). Reversing the order of variables added revealed that depression remained significant; $\mathrm{r}^{2}$ change $=.15, F(1,70)=17.09, p<.001$; BDI-II $(\mathrm{b}=.45, p<.001) \mathrm{OCD}(\mathrm{b}=.18, p=.15) ; \mathrm{GD}(\mathrm{b}=-.15, p=.25), \mathrm{HD}(\mathrm{b}=.20, p=.191)$, gender $(\mathrm{b}=.004, p=.972)$, or age $(\mathrm{b}=-.27, p=.018)$.

\section{Perfectionism.}


For the analyses of the self-report perfectionism data, as measured by the Frost Multidimensional Perfectionism Scale, no cases were excluded. The a priori significance level was calculated using the Holm's procedure to account for multiple comparisons at $0.05, .025$, $.016 ; .013$ Data met the assumptions of normality. The data violated the homogeneity of variance test, $F(3,73)=3.34, p=.024$. Therefore, Welch's $\mathrm{F}$ was reported. The ANOVA revealed that group was not significantly associated with the total perfectionism score, Welch's $F(3,36.84)=$ $1.98, p=.134, \eta^{2}=.084$. Examination of the subscales revealed significant group differences on the Personal Standard subscale, $F(3,73)=4.65, p=.005, \eta^{2} \mathrm{p}=.161$, and the Concern about Mistakes/Doubts subscale, $F(3,73)=4.57, p=.005, \eta_{\mathrm{p}}^{2}=.158$. Tukey post hoc analyses on the significant Personal Standard ANOVA data indicated that those in the HD group had significantly lower levels of personal standard perfectionism than the controls $(p=.032)$, the OCD group $(p=.003)$, and the GD group $(p=.05)$. Tukey post hoc analyses on the Control of Mistakes/Doubts ANOVA data indicated that those in the OCD group had significantly more perfectionism in this domain than controls $(p=.003)$. No significant group differences emerged on the Parental Expectations and Criticism subscale, $F(3,73)=2.22, p=.092, \eta^{2} \mathrm{p}=.084$.

A follow-up hierarchical regression that included the demographic variables (age and gender) in the model revealed that group remained a significant predictor of the subscale Personal Standard when age and gender were included in the model, $\mathrm{r}^{2}$ change $=.06 ., F(2,74)=$ $2.45, p=.093$. More specifically, in step 1 of this hierarchical regression, age and gender were entered, neither age $(b=-.11, p=.344)$ nor gender $(b=-.21, p=.072)$ contributed significant variance to the model. In step 2 of this hierarchical regression, groups were entered, and contributed significant variance, $\mathrm{r}^{2}$ change $=.12, F(3,71)=3.61, p=.017$; gender $(\mathrm{b}=-.17, p=$ 
$.168)$, age $(\mathrm{b}=.07, p=.564), \mathrm{OCD}(\mathrm{b}=.14, p=.294), \mathrm{GD}(\mathrm{b}=.08, p=.588), \mathrm{HD}(\mathrm{b}=-.32, p=$ $.041)$.

A second hierarchical regression that included both the demographic (age, gender) variables and the measure of depressive symptoms (BDI-II) revealed that group continued to add significant, variance when depressive symptoms were included in the model; $r^{2}$ change $=.16$, $F(3,70)=5.05, p=.003 ; \mathrm{BDI}-\mathrm{II}(\mathrm{b}=.31, p=.011), \mathrm{OCD}(\mathrm{b}=-.01, p=.923), \mathrm{GD}(\mathrm{b}=.00, p$ $=.991), \mathrm{HD}(\mathrm{b}=-.50, p=.003)$, gender $(\mathrm{b}=-.17, p=.152)$, age $(\mathrm{b}=.10, p=.418)$.

A follow-up hierarchical regression that included the demographic variables (age and gender) in the model revealed that group remained a significant predictor of Concern about Mistakes/Doubts subscale the subscale when age and gender were included in the model, $\mathrm{r}^{2}$ change $=.02, F(2,74)=.85, p$ $=.431$. More specifically, in step 1 of this hierarchical regression, age and gender were entered, neither age $(\mathrm{b}=-.12, p=.312)$ nor gender $(\mathrm{b}=-.08, p=.517)$ contributed significant variance to the model. In step 2 of this hierarchical regression, groups were entered, and contributed significant variance, $\mathrm{r}^{2}$ change $=.18, F(3,71)=5.46, p=.002$; gender $(\mathrm{b}=-.16, p=.200)$, age $(\mathrm{b}=-.20, p=.116)$, OCD $(\mathrm{b}=.52, p<$ $.001), \mathrm{GD}(\mathrm{b}=.23, p=.099), \mathrm{HD}(\mathrm{b}=.33, p=.032)$.

A second hierarchical regression that included both the demographic (age, gender) variables and the measure of depressive symptoms (BDI-II) revealed that group did not add significant variance when depressive symptoms were included in the model; $r^{2}$ change $=.06$, $F(3,70)=2.18, p=.098$. Although the overall groups did not add significant variance, the OCD group alone continued to predict significant variance; BDI-II $(\mathrm{b}=.44, p<.001)$, OCD $(\mathrm{b}=.31, p$ $=.022), \mathrm{GD}(\mathrm{b}=.13, p=.329), \mathrm{HD}(\mathrm{b}=.08, p=.620)$, gender $(\mathrm{b}=-.16, p=.166)$, age $(\mathrm{b}=-.16$, $p=.160)$. 
Table 4

Dysfunctional belief measures of patients with obsessive-compulsive disorder (OCD), hoarding disorder (HD), a grooming disorder (GD), and healthy controls $(H C)$

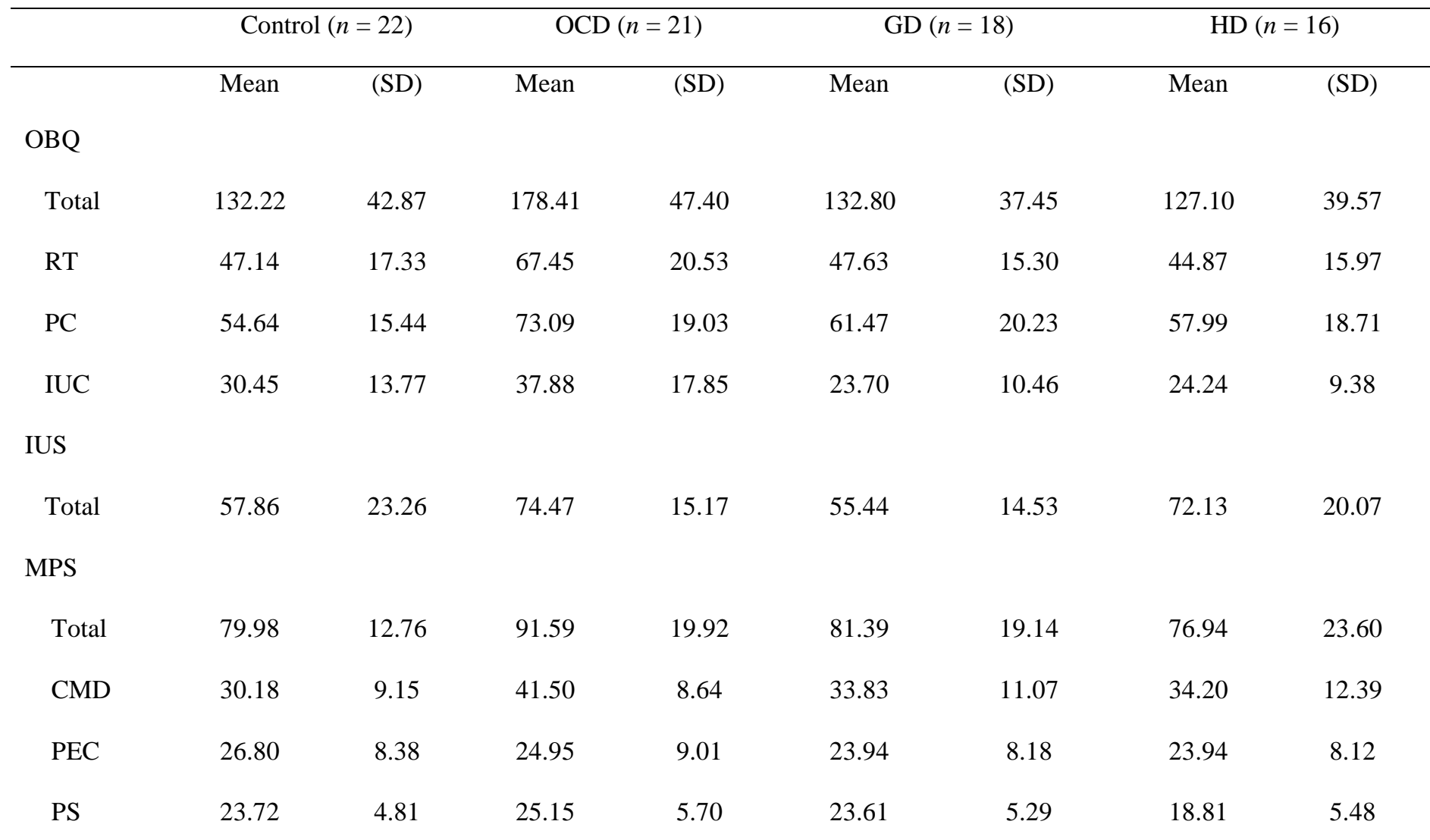

Note. $\mathrm{M}=$ Mean; $\mathrm{SD}=$ Standard deviation; $\mathrm{OCD}=$ obsessive-compulsive disorder; $\mathrm{GD}=$ grooming disorder; $\mathrm{HD}=$ hoarding disorder; $\mathrm{OBQ}=$ Obsessional Beliefs Questionnaire; $\mathrm{RT}=$ Responsibility/Threat Subscale; $\mathrm{PC}=$ Perfectionism/Control Subscale; IUC= Intolerance of Uncertainty Subscale; IUS = Intolerance of Uncertainty Scale; MPS $=$ Multidimensional Perfectionism Scale CMD = concern over mistakes and doubts $;$ PEC $=$ parental expectations and criticism; $\mathrm{PS}=$ personal standards; $\mathrm{O}=$ organization 


\section{Associated Constructs}

See table 5.0 for means and standard deviations of the associated construct measures.

\section{Indecisiveness.}

For the analyses of the self-report indecision data, as measured by the Indecisiveness Scale, no cases were excluded. The a priori significance level was calculated using the Holm's procedure to account for multiple comparisons at 0.05 . The data violated the homogeneity of variance test, $F(3,73)=2.84, p=.045$. Therefore, Welch's F was reported. The ANOVA revealed that group was significantly associated with indecision, Welch's $F(3,38.5)=8.21, p<$ $.001, \eta_{\mathrm{p}}^{2}=.29$. Games-Howell was employed as a post-hoc test, which revealed that those in the $\operatorname{OCD}(p=.002)$ and HD $(p=.001)$ groups reported significantly more indecision than controls. Those in the HD group also reported significantly more indecision than those in the GD groups $(p=.04)$.

A follow-up hierarchical regression that included the demographic variables (age and gender) in the model revealed that group remained a significant predictor of self-report indecision when age and gender were included in the model, $\mathrm{r}^{2}=.34, F(3,71)=11.50, p<.001$. More specifically, in step 1 of this hierarchical regression, age and gender were entered, and neither age $(b=.14, p=.23)$ nor gender $(b=.03, p=.826)$ predicted self-report indecision. When groups were added in the second step of the model, group remained significant predictor of variance. More specifically, in step 2 of this hierarchical regression, groups were entered, and all three clinical groups contributed significant variance to the model; OCD $(b=.49, p<.001)$ $\mathrm{HD}(\mathrm{b}=.75, p<.001), \mathrm{GD}(\mathrm{b}=.26, p=.034)$.

A second hierarchical regression that included both the demographic (age, gender) variables and the measure of depressive symptoms (BDI-II) revealed that group remained a 
significant predictor of self-report indecision, $\mathrm{r}^{2}=.44, \mathrm{~F}(3,70)=5.0, p=.003$; in the second step of this model, age $(\mathrm{b}=-.22, p=.037)$, BDI-II $(\mathrm{b}=.56, p<.001)$, OCD $(\mathrm{b}=.31, p=.013)$, and HD $(b=.53, p<.001)$, but not gender $(b=-.023, p=.825)$ or GD $(b=.19, p=.13)$, contributed significant variance to the model.

\section{Impulsivity.}

For the analyses of the self-report impulsivity data, as measured by the Barrett Impulsiveness Scale (BIS), no cases were excluded. The a priori significance level was calculated using the Holm's procedure to account for multiple comparisons at $0.05, .025, .016$; .013. Data met the assumptions of normality, such that the data distribution approximated normality and the variances did not differ significantly across groups. ANOVA revealed no significant association between group and total BIS scores, $F(3,73),=.65, p=.586, \eta^{2} \mathrm{p}=.03$. Examination of the subscales revealed no significant group differences on the attention, motor, or non-planning subscales; $F(3,73)=2.40, p=.075, \eta_{\mathrm{p}}^{2}=.09, F(3,73)=1.38, p=.256, \eta_{\mathrm{p}}^{2}=$ $.05, F(3,73)=.16, p=.93, \eta^{2}{ }_{p}=.01$. 
Table 5

Associated construct measures of patients with obsessive-compulsive disorder (OCD), hoarding

disorder (HD), a grooming disorder (GD), and healthy controls $(H C)$

\begin{tabular}{|c|c|c|c|c|c|c|c|c|}
\hline & \multicolumn{2}{|c|}{ Control $(n=22)$} & \multicolumn{2}{|c|}{$\mathrm{OCD}(n=21)$} & \multicolumn{2}{|c|}{$\mathrm{GD}(n=18)$} & \multicolumn{2}{|c|}{$\mathrm{HD}(n=16)$} \\
\hline & Mean & (SD) & Mean & (SD) & Mean & (SD) & Mean & (SD) \\
\hline \multicolumn{9}{|l|}{ IS } \\
\hline Total & 35.95 & 9.85 & 47.53 & 9.75 & 42.65 & 6.67 & 52.63 & 12.34 \\
\hline \multicolumn{9}{|l|}{ BIS } \\
\hline Total & 60.85 & 12.49 & 63.40 & 12.98 & 65.40 & 8.73 & 61.66 & 7.21 \\
\hline AT & 15.86 & 4.54 & 18.62 & 4.36 & 17.26 & 3.65 & 15.60 & 2.89 \\
\hline MO & 20.97 & 3.58 & 20.57 & 4.17 & 23.09 & 5.08 & 21.93 & 3.90 \\
\hline NP & 24.02 & 5.87 & 24.21 & 6.16 & 25.04 & 3.82 & 24.13 & 3.56 \\
\hline
\end{tabular}

Note. $\mathrm{M}=$ Mean; $\mathrm{SD}=$ Standard deviation; $\mathrm{OCD}=$ obsessive-compulsive disorder GD = grooming disorder $; \mathrm{HD}=$ hoarding disorder; IS = Indecisiveness Scale; BIS = Barratt Impulsivity Scale Version 11; AT = attention subscale; $\mathrm{MO}=$ motor subscale $; \mathrm{NP}=$ non-planning subscale 


\section{Quality of Life.}

See table 6.0 for means and standard deviations of the QOL measures. For the analyses of the self-report QOL data, as measured by the Quality of Life Enjoyment and Satisfaction Questionnaire - Short Form, no cases were excluded. The a priori significance level was calculated using the Holm's procedure to account for multiple comparisons at 0.05 . Data met the assumptions of normality. ANOVA revealed a significant association between group and QLESSF scores, $F(3,73)=11.47, p<.001, \eta^{2} \mathrm{p}=.32$. Tukey post hoc analyses on the ANOVA data indicated that those in the OCD and HD group had significantly impaired QOL compared to controls $(p=.004 ; p<.001$, respectively). Those in the HD group also had significantly impaired QOL compared to those in the GD group $(p=.001)$. Those in the GD group did not significantly differ from controls $(p=.14)$.

A follow-up hierarchical regression that included the demographic variables (age and gender) in the model revealed that group remained a significant predictor of QOL when age and gender were included in the model, $\mathrm{r}^{2}=.33 ., F(3,71)=9.51, p<.001$. More specifically, in step 1 of this hierarchical regression, age and gender were entered, neither age $(b=-.19 ., p=.094)$ nor gender $(b=-.097, p=.397)$ contributed significant variance to the model. In step 2 of this hierarchical regression, groups were entered, the OCD group $(b=-.42, p=.001)$ and the HD group $(b=-.67, p<.001)$ contributed significant variance to the model, while age $(b=.07 ., p=$ $.527)$, gender $(\mathrm{b}=.02 ., p=.852)$, and GD $(\mathrm{b}=-.17, p=.205)$ did not.

A second hierarchical regression that included both the demographic (age, gender) variables and the measure of depressive symptoms (BDI-II) revealed that while the overall model remained significant, group continued to provide significant variance when depressive symptoms were included in the model, $\mathrm{r}^{2}=.47, F(3,70)=3.58, p=.018$; BDI-II $(\mathrm{b}=-.45, p<.001)$, 
OCD $(b=-.20, p=.087), \mathrm{GD}(\mathrm{b}=-.06, p=.627), \mathrm{HD}(\mathrm{b}=-.41, p=.004)$, gender $(\mathrm{b}=.02, p=$ $.831)$, or age $(b=.04, p=.728)$.

For the analyses of the self-report QOL data, as measured by the Sheehan Disability Scale, no cases were excluded. The a priori significance level was calculated using the Holm's procedure to account for multiple comparisons at $0.05, .025, .016 ; .013$. Data met the assumptions of normality. ANOVA revealed a significant association between group and total SDS scores, $F(3,73)=15.36, p<.001, \eta^{2} \mathrm{p}=.39$. Tukey post hoc analyses on the ANOVA data indicated that controls were significantly less impaired on QOL compared to all three clinical groups, OCD $(p<.001)$; GD $(p<.001)$; HD $(p<.001)$. No significant differences emerged between the clinical groups.

A follow-up hierarchical regression that included the demographic variables (age and gender) in the model revealed that group remained a significant predictor of QOL when age and gender were included in the model, $\mathrm{r}^{2}=.39, F(3,71)=13.83, p<.001$. More specifically, in step 1 of this hierarchical regression, age and gender were entered, neither age $(\mathrm{b}=.18 ., p=.13)$ nor gender $(b=-.07, p=.54)$ contributed significant variance to the model. In step 2 of this hierarchical regression, groups were entered, all three clinical groups contributed significant variance to the model: $\mathrm{OCD}(\mathrm{b}=.68, p<.001), \mathrm{GD}(\mathrm{b}=.46, p<.001)$, and HD group $(\mathrm{b}=.63, p$ $<.001)$, while age $(b=-.10 ., p=.362)$, and gender $(b=-.01, p=.90)$ did not .

A second hierarchical regression that included both the demographic (age, gender) variables and the measure of depressive symptoms (BDI-II) revealed that group contributed significant variance even when depressive symptoms were included in the model. $\mathrm{r}^{2}=.52, F(3$, $70)=6.70, p<.001$; gender $(b=-.01, p=.882)$, age $(b=-.07, p=.504)$, BDI-II $(b=.41, p<$ $.001)$, OCD $(b=.48, p<.001), \mathrm{GD}(\mathrm{b}=.36, p=.002), \mathrm{HD}(\mathrm{b}=.39, p=.004)$. 
Examination of the work/school subscale of the SDS scale revealed a significant group difference, $F(3,73)=8.25, p<.001, \eta^{2} \mathrm{p}=.25$. Tukey post hoc analyses on the ANOVA data indicated that those in the OCD group had significantly more impairment in work/school than controls $(p<.001)$. Those in the GD group and HD group did not significantly differ on work/school impairment compared to controls $(p=.052 ; p=.092)$.

Examination of the social life/leisure activities subscale of the SDS revealed a significant group difference, $F(3,73)=9.87, p<.001, \eta^{2} \mathrm{p}=.29$. Tukey post hoc analyses on the ANOVA data indicated that all three clinical groups were impaired socially compared to controls; OCD ( $p$ $<.001), \mathrm{GD}(p<.01), \mathrm{HD}(p<.001)$. No significant group differences emerged between the clinical groups.

Examination of the family life/home responsibilities subscale of the SDS revealed a significant group difference, $F(3,73)=18.56, p<.001, \eta_{\mathrm{p}}^{2}=.43$. Tukey post hoc analyses on the ANOVA data indicated that all three clinical groups were impaired in family life/home responsibility compared to controls; OCD $(p<.001)$, GD $(p<.001)$, $\mathrm{HD}(p<.001)$. No significant group differences emerged between the clinical groups. 
Table 6

Quality of life measures of patients with obsessive-compulsive disorder (OCD), hoarding

disorder (HD), a grooming disorder (GD), and healthy controls (HC)

\begin{tabular}{|c|c|c|c|c|c|c|c|c|}
\hline & \multicolumn{2}{|c|}{ Control $(n=22)$} & \multicolumn{2}{|c|}{$\mathrm{OCD}(n=21)$} & \multicolumn{2}{|c|}{$\mathrm{GD}(n=18)$} & \multicolumn{2}{|c|}{$\mathrm{HD}(n=16)$} \\
\hline & Mean & $(\mathrm{SD})$ & Mean & (SD) & Mean & $(\mathrm{SD})$ & Mean & (SD) \\
\hline \multicolumn{9}{|l|}{ QLES } \\
\hline Raw Total & 53.48 & 8.83 & 45.25 & 7.28 & 50.28 & 5.39 & 39.70 & 8.42 \\
\hline $\begin{array}{l}\text { Percentage } \\
\text { Max. }\end{array}$ & .71 & .16 & .56 & .13 & .65 & .10 & .46 & .15 \\
\hline
\end{tabular}

SDS

$\begin{array}{ccccccccc}\text { Total } & 3.45 & 5.03 & 15.98 & 6.51 & 12.33 & 7.01 & 15.18 & 8.45 \\ \text { Work } & 1.28 & 1.80 & 5.74 & 3.40 & 3.72 & 2.94 & 3.57 & 3.53 \\ \text { Social/leisure } & 1.27 & 2.00 & 4.86 & 2.33 & 4.44 & 2.85 & 5.61 & 3.83 \\ \text { Home/family } & 0.91 & 1.50 & 5.38 & 2.27 & 4.16 & 2.62 & 5.99 & 3.10\end{array}$

Note. $\mathrm{M}=$ Mean; $\mathrm{SD}=$ Standard deviation; $\mathrm{OCD}=$ obsessive-compulsive disorder; GD = grooming disorder; HD = hoarding disorder; QLES = Quality of Life Enjoyment and Satisfaction Questionnaire- Short Form; SDS = Sheehan Disability Scale 


\section{Neurocognition, dysfunctional beliefs, and QOL.}

Correlations are displayed in table 7.0. In an exploratory analysis to examine the relationship between neurocognition, dysfunctional beliefs, and QOL, Pearson correlations were computed between the dependent variables for the neurocognitive tasks, dysfunctional beliefs, and QOL scores (as measured by the QLES and the SDS) for clinical participants (controls were excluded for the purpose of this analysis). Due to the vast number of correlations computed, a conservative a priori significance level of .01 was utilized to account for multiple tests. Dependent variables of the WCST (total errors, $r=-.32, p=.005$; nonperseverative errors, $r=$ $.29, p=.011$; trials administered, $r=-.34, p=.003$ ), TMT (number/sequence $r=.30, p=.008$; letter/number sequence, $r=-.36, p=.002$ ), RCFT (copy, $r=.37, p=.006$; immediate recall, $r=$ $.41, p=.003$; delayed recall, $r=.36, p=.010$ ), and the digit symbol (number completed, $r=.33$, $p=.009)$ were all significantly correlated with QOL, as measured by the QLES..

Examination of dysfunctional beliefs and QOL revealed that total scores on the IUS ( $r=$ $.50, p<.001)$, BIS $(r=-.43, p<.001)$, and IS $(r=-.63, p<.001)$ were significantly correlated with the QLES. The same dysfunctional beliefs were significantly correlated with the SDS; IUS $(r=.41, p<.001)$, BIS $(r=.35, p=.002)$, and IS $(r=.56, p<.001)$. 


\section{Results Summary of Significant Findings}

\section{Neurocognition.}

The following were significant findings;

1) The SSRT significantly differed between groups on the stop-signal task, with those in the HD group having a longer SSRT than controls. The HD group continued to predict significant variance when age, gender, and depressive symptoms were added to the model.

2) The WCST number of trials administered and total correct response significantly differed between groups. Those in the HD group had more trials administered and fewer total correct response than controls. Those in the HD group also had significantly more trials administered than those in the GD group. As these analyses violated parametric assumptions and nonparametric statistics were used, hierarchical regression could not be used to examine additional sources of variance.

3) For the TMT, both number sequencing and letter/number sequencing significantly differed between groups. Those in the OCD and HD groups took significantly longer than controls on number sequencing. For letter/number sequencing, those in the HD group took significantly longer than controls. When age, gender, and depressive symptoms were included in the model, OCD continued to predict significant variance in number sequencing but group no longer contributed significant variance to letter/number sequencing.

4) For the RCFT, all three primary variables (copy, immediate recall, and delayed recall) significantly differed between groups. For the copy variable, the HD and GD groups had significantly lower copy scores than controls. Those in the OCD group had 
significantly higher copy scores than those in the GD group and the HD group. As nonparametric statistics were employed, hierarchical regression could not be used to examine the effects of age, gender, and depressive symptoms. For immediate recall, those in the GD and HD group recalled less than controls. For the delayed recall variable, those in the HD group recalled significantly less than controls. Group no longer predicted significant variance in the immediate or delayed recall when age, gender, and depressive symptoms were included in the model.

\section{Dysfunctional Beliefs.}

1) $\mathrm{OBQ}$ total score; The OCD group had significantly elevated beliefs compared to controls and both other clinical groups. The OCD group remained a significant predictor of variance when age, gender, and depressive symptoms were entered into the model.

a. Responsibility/Threat subscale; the OCD group had significantly elevated beliefs compared to controls and both other clinical groups. The OCD group remained a significant predictor of variance when age, gender, and depressive symptoms were entered into the model.

b. Perfectionism/IC subscale; The OCD group had significantly elevated beliefs compared to controls, but were not significantly different than the other clinical groups. The OCD group remained a significant predictor of variance when age, gender, and depressive symptoms were entered into the model.

c. Importance/Control of Thoughts subscale; the OCD group had significantly elevated beliefs compared to both clinical groups. The OCD 
group did not remain a significant predictor of variance when age, gender, and depressive symptoms were entered into the model.

2) Intolerance of Uncertainty Scale; The OCD group had significantly elevated beliefs compared to controls and the GD group. The HD group did not significantly differ from OCD, but did have significantly elevated beliefs compared to the GD group. Group no longer predicted significant variance when age, gender, and depressive symptoms were entered into the model.

3) Frost Multi-dimensional Perfectionism Scale; no significant group differences on the total score.

a. Personal Standards subscale, those in the HD group had significantly lower scores compared to controls and both clinical groups. The HD group remained a significant predictor of variance when age, gender, and depressive symptoms were entered into the model.

b. Concern about Mistakes/Doubts subscale; The OCD group reported significantly elevated beliefs compared to controls. There was no significant difference between the OCD group and other clinical groups. OCD group remained a significant predictor of variance when age, gender, and depressive symptoms were entered into the model.

\section{Associated Construct.}

1) Indecisiveness Scale; Those in the OCD and HD groups reported significantly more indecision than controls. Group remained a significant predictor of variance when age, gender, and depressive symptoms were entered into the model 


\section{QOL}

1) QLES-SF; Those in the OCD and HD groups reported significantly worse QOL than controls. Those in the HD group reported significantly worse QOL than those in the GD group.

2) SDS; All three clinical groups reported significant impairment compared to controls.

a. Work/School subscale; Those in the OCD group were significantly more impaired than controls.

b. Social Life/Leisure subscale; All three clinical groups reported significant impairment compared to controls.

c. Family Life/Home Responsibilities subscale; All three clinical groups reported significant impairment compared to controls.

3) Examination of the relationship between CEs and QOL revealed significant correlations with some neurocognitive dependent variables (WCST, TMT, RCFT, digit symbol), and nearly all dysfunctional belief variables (IUS, BIS, IS) with the QLES. No neurocognitive, but all dysfunctional belief variables, were associated with QOL measured using the SDS. 


\section{Discussion}

The primary purpose of this dissertation was to examine candidate endophenotypes across the OCRDs from two theoretical orientations of OCD etiology with robust empirical support (corticostriatal-thalamic model and the learning model). In addition to neurocognitive variables, contemporary characterizations of endophenotypes (see Taylor, 2012) suggest that dysfunctional beliefs are promising endophenotypes of OCD. The current study examined the six categories of beliefs reliably linked to OCD; inflated personal responsibility, overestimation of threat, perfectionism, IU, overimportance of thoughts, and need to control thoughts (e.g., Clark, 2004; Frost \& Steketee, 2002; Salkovskis, 1996; OCCWG, 1997; OCCWG, 2005) across the OCD spectrum, and measured several of these constructs multidimensionally.

This dissertation sought to examine (preliminarily) how narrow or broad common CEs are by examination of these constructs across the OCRD spectrum. DSM-5's categorization of these disorders within the OCD spectrum suggests that these conditions may share more general etiological factors (e.g., candidate endophenotypes) than other disorders. Work examining the specificity of endophenotypes is in its infancy; however, the importance of determining specificity has been highlighted in review of OCD endophenotypes (Taylor, 2012). In exploratory analyses, this dissertation also examined candidate endophenotypes as predictors of QOL, and examined both self-reported indecision and impulsivity across the spectrum.

A review of the neurocognitive endophenotype literature revealed that neurocognitive research within OCD has been fraught with inconsistent findings and highlighted the dearth of research in the related disorders. A review and meta-analysis of the current state of the neurocognitive literature in OCD has highlighted that neuropsychological factors, clinical factors, and general factors are negatively influencing the replicability of findings (Abramovitch 
et al. 2015; Kuelz et al., 2004). Such factors include, but are not limited to; issues with multiple comparison, comorbidity of depressive symptoms, the inclusion of hoarding as a symptom of OCD, failure to report recruitment source, symptom severity, and education level, and the heterogeneous nature of OCD (Abramovitch et al. 2015; Kuelz et al., 2004).

The current study incorporated some of the recommendations made in critical reviews into the current research design. The current study sought to address the limitations of previous research by addressing multiple comparisons using the Holm procedure (Holm, 1979) for the majority of analyses or setting a conservative significance threshold for others, reporting symptom severity and education, and measuring and including depressive symptoms in the model (although this is a contentious issue in the literature, see methods and limitations for a more fulsome discussion). Despite efforts to avoid common pitfalls, the current study had significant limitations including insufficient power, inflated type 1 error despite using the Holm's procedure, inability to account for the heterogeneous nature of OCD, and although symptom severity was measured, it was not included in analyses. The results of the current study should be interpreted with these shortcomings in mind and particular attention should be given to the limitation sections below which elaborates on each of these shortcomings.

Due to the aforementioned difficulties of the current empirical literature, the current dissertation did not test specific a priori hypotheses (please see current study section for more information regarding the rationale for this approach). Broadly, the dissertation aimed to address the following questions about candidate endophenotypes in the OCRDs (1) what is the specificity of neurocognitive and dysfunctional belief candidate endophenotypes across the OCRDs? It was predicted that there would be general endophenotypes underlying all of the OCRDs, and that there would be some specific CEs linked to some OCRDs but not others. (2) Is 
self-report impulsivity correlated with the OCRDs? It was predicted that impulsivity would be associated with the related disorders, but not OCD; (3) Is there an association between candidate endophenotypes and QOL? It was predicted that some CEs would be associated with impairments in QOL. The results from each inquiry are discussed below.

(1a) What is the specificity of neurocognitive candidate endophenotypes across the OCRDs?

\section{Inhibitory dysfunction}

Results revealed that those with HD had significantly longer stop-signal reaction times compared to controls, and those in all other clinical groups, indicating a greater degree of difficulty inhibiting a motor response. The findings of this study support the work done suggesting that those with HD perform worse than controls on measures of response inhibition (Morein-Zamir et al., 2014; Rasmussen, 2012). However, the lack of a significant association between response inhibition and the OCD group is contrary to some other previous studies, including a meta-analysis that found a medium effect-size deficit in action cancellation (as measured by the Stop Signal Task) (Lipszyc \& Schachar, 2010). Nevertheless, numerous individual studies have similarly failed to find an association between OCD and response inhibition (Boone et al., 1991; Kalanthroff et al., 2016; Krishna et al., 2011; Rao et al., 2008). Likewise, although the bulk of research suggests a deficit in response inhibition in the GDs (e.g., Grant et al., 2011, Odlaug et al., 2010; Odlaug et al., 2013), not all studies have found this deficit (Grant et al., 2011; Snorrason et al., 2011).

To make sense of this finding, closer examination of the group means revealed that the control group in this study performed worse than in other published studies and, in fact, the OCD participants' SSRT was on-par with other published work finding impairment (e.g., MoreinZamir et al., 2014). Thus, it is possible that under-performance by the control group contributed 
to this null result and may speak to motivation/effort in the control group. A lack of a significant finding in the OCD and GD groups may also reflect this study's limited power.

Taken together, it is difficult to ascertain the specificity of inhibitory dysfunction due to the significant power limitations of this study and similar shortcomings in much of the aforementioned cited work. While the current study suggests specificity to HD, it becomes apparent when reviewing the bulk of the literature that it is likely that OCD (see meta-analysis Lipszyc \& Schachar, 2010) and the GDs (Grant et al., 2011, Odlaug et al., 2010; Odlaug et al., 2013) share inhibitory dysfunction deficits.

\section{Interference control}

No significant differences emerged on the Stroop task. All clinical groups and controls had T-scores above 40, which is considered "normal" (Golden, 1976). This is discrepant from the findings in OCD, which have revealed increased reaction times on incongruent trials (Bannon, Gonsalvez, Croft, \& Boyce, 2002; Nabeyama et al., 2008; Nakao et al., 2009; Penades et al., 2007; Schlosser et al., 2010). Thus, it is possible that this sample of OCD patients out-performed what is typically seen on this task in OCD, and raises the possibility of differing sample characteristics (e.g., higher education level) than in previous work. The lack of a significant finding may also be a result of this study's limited power. In keeping with previous literature (Sumner et al., 2016; Tolin et al., 2011), no performance deficit was observed in HD compared to controls. The lack of a significant finding between GD and Stroop performance is in keeping with some literature that failed to find a significant association (e.g., Coetzer \& Stein, 1999).

While the current study suggests no impairment in interference control across the

OCRDs, synthesis of previous literature suggests impairment in OCD (e.g., Bannon et al., 2002; Nabeyama et al., 2008; Nakao et al., 2009; Penades et al., 2007; Schlosser et al., 2010) but not in 
HD (e.g., Sumner et al., 2015; Tolin, Villavicencio et al., 2011) or GD (e.g., Coetzer \& Stein, 1999). Additional studies, particularly in the related-disorders, are necessary to further elucidate the specificity of this domain.

\section{Set-shifting}

Results revealed that, for the WCST, the number of trials administered and the number of total correct responses were significantly more impaired in HD compared to controls. This is consistent with previous literature (Ayers et al., 2013; McMillan et al., 2013; Morein-Zamir et al., 2014) which suggests that set-shifting may be impaired in HD. Those with OCD did not significantly differ from either controls or other clinical groups. This adds to the conflicting literature, with approximately half of studies suggesting no impairment in OCD (Abbruzzese, Bellodi et al., 1995; Abbruzzese, Ferri et al., 1995; Abbruzzese et al., 1997; Deckersbach et al., 2000; Gross-Isseroff et al., 1996; Laniti, 2005; Moritz et al., 2001, 2002; Zielinski et al., 1991). The lack of a finding in the GD group in this domain is also consistent with previous literature (Bohne et al., 2005; Chamberlain et al., 2006; Grant et al., 2011; Odlaug et al., 2010).

For the Intradimensional/Extradimensional Task, no significant differences emerged. Interestingly, in keeping with previous literature, when set-shifting in HD was examined using the IE-ED, no differences between hoarding patients and controls have emerged (de Kort, 2012; Grisham, 2010), despite the significant findings using the WCST. This speaks to the possibility that set-shifting may be a heterogeneous construct with both tasks tapping into slightly different constructs.

Synthesis of the set-shifting literature reveals conflicting findings across individual studies, but several meta-analyses suggest impaired set-shifting in OCD (Abramovitch et al., 2013; Henry, 2006). The bulk of the literature, including this study, suggest a deficit in set-shifting in 
HD (e.g., Ayers et al., 2013; McMillan et al.,, 2013; Morein-Zamir et al., 2014).There has been a fairly consistent null finding for the GDs (e.g., Bohne et al., 2005; Chamberlain et al., 2006;

Grant et al., 2011; Odlaug et al., 2010), as was the case in the current study.

\section{Non-verbal memory}

Results suggest that those in the HD and GD groups had significantly lower copy scores than controls. The copy score is thought to be a measure of executive functioning, rather than memory (Weber, Riccio, \& Cohen, 2013). Additionally, those in the HD and GD groups had significantly impaired immediate and delayed recall; however, these results were no longer statistically significant when age, gender, and depressive symptoms were included in the model. Nevertheless, this data trend is consistent with the literature suggesting that patients with HD show deficits in non-verbal memory (Hartl et al., 2004; Testa et al., 2011).

Given a recent meta-analysis that revealed a large effect size when examining non-verbal memory impairment in OCD (Cohen's $d=.76$; Abramovitch et al., 2013), the lack of a statistically significant difference between OCD and controls is surprising. However, there have been other individual studies (Bédard et al., 2009; Hanes, 1998; Simpson et al., 2006) that have failed to find a significant association between OCD and non-verbal memory as measured by the RCFT. This highlights the replication challenges in the neurocognitive literature, and speaks to difficulties with power, symptom heterogeneity, and sample representativeness in individual studies.

Similarly, examination of the SWM data revealed a non-significant trend, such that those with HD had impaired performance in terms of errors and strategy compared to controls. Closer examination of the group means revealed that the control group in this study performed worse than in other published studies (e.g., Dunai et al., 2010). Thus, once again, it is possible that 
under-performance by the control group contributed to this largely non-significant result, despite the numerous between-search errors made by the HD group and, to a lesser extent, the OCD group. Additionally, the current study's limited power could have contributed to the absence of a positive finding, particularly with a trending result.

The specificity of nonverbal memory impairment across the OCRDs is difficult to ascertain with discrepant results across previous work, including in the current study.

Nevertheless, a meta-analysis revealed non-verbal memory impairment in OCD (Abramovitch et al., 2013). In HD, approximately half of studies have found poorer recall in HD compared to controls (e.g., Hartl et al., 2004; Testa et al., 2011), while others have not (e.g., Sumner et al., 2016; Tolin, Meunier et al., 2011). Similar mixed findings have been found for the GDs (e.g., Bohne et al., 2005; Chamberlain, Fineberg, Menzies et al., 2007; Keuthen et al., 1996). Additional research is necessary that takes into consideration organizational strategy and possible confounding variables (such as symptom severity) to clarify these conflicting results.

\section{Verbal memory}

No significant differences emerged between any of the clinical groups and the healthy controls on verbal memory. This finding is in keeping with previous research suggesting that those with related disorders do not exhibit verbal memory impairment (Bohne et al., 2005; Fitch, 2011; Keuthen et al., 1996; Sumner et al., 2016; Tolin et al., 2011). Findings on verbal memory from individual studies in OCD have been mixed, with several studies suggesting no verbal memory impairment in OCD (Bédard et al., 2009; Bohne et al., 2005; Boone et al., 1991; Burdick et al., 2008; Christensen et al., 1992;; deGeus et al., 2007; Martin et al., 1995; MataixCols et al., 1999; Zielinski et al., 1991). A recent meta-analysis, however, found a small effect size for decreased verbal memory in OCD patients compared to controls, Cohen's $d=.33$ 
(Abramovitch et al., 2013). Given the small effect size of this deficit, it is also possible that null findings in individual studies may be a result of insufficient power. Taken together, results of the previous literature suggest some specificity of impaired verbal memory performance to $\mathrm{OCD}$, while the related disorders remain intact.

There has been some suggestion that it may be that organizational strategy in the form of semantic clustering (e.g., grouping words of similar categories to assist with retention and recall), and not memory per se, may be impaired in OCD (e.g., Cabrera et al., 2001); however, this hypothesis has not been supported by other findings (e.g., de Geus et al., 2007) that have reported nearly identical semantic clustering between OCD groups and controls. The current research did not find a statistically significant difference between any of the clinical groups or non-psychiatric controls on semantic clustering.

(1b) What is the specificity of dysfunctional belief candidate endophenotypes across the OCRDs?

Those in the OCD group had significantly inflated beliefs when the total score, responsibility/threat, and over-importance of thoughts were examined compared to controls and the other clinical groups on the OBQ. This suggests specificity of Responsibility/Threat beliefs and Importance/Control of Thoughts beliefs to OCD. The specificity of Perfectionism/IU beliefs showed differing patterns depending on the measure utilized to measure the construct. For example, perfectionism/IUC measured using subscales of the OBQ produced different results than using the FMPS or the IUC scale. Overall Perfectionism/IU beliefs appear to be broad CEs implicated across disorders for the following reasons: When measured using the subscale on the OBQ, the OCD group did not statistically differ from the other clinical groups. All three clinical groups showed higher levels of perfectionism/IU compared to controls. 
When IU was measured using the IUS, there appeared to be specificity for OCD and HD, as the OCD group had significantly elevated beliefs compared to controls and the GD group. The HD group did not significantly differ from OCD, but did have significantly elevated beliefs compared to the GD group. This is consistent with the phenomenology of certain subtypes of OCD (e.g., checking and repeating compulsions), and the theory that individuals with OCD find uncertainty to be distressing and engage in compulsions in an attempt to gain certainty (Carr, 1974; Guidano \& Liotti, 1983). Previous research has found that those with HD showed comparable rates of IU to those with OCD or Generalized Anxiety Disorder, and significantly higher levels compared to controls (Wheaton et al., 2016). In the current study, the HD group showed elevated levels compared to the GD group. Examination of the group means revealed that the control group reported higher levels of IUS than the GD group. Thus, the slightly elevated level of IUS in the control group may have contributed to the non-significant finding between the HD group and controls.

When depressive symptoms were included in the model, however, groups were no longer related to IUC, regardless of the order in which depression was added to the model. This finding is in-line with recent research suggesting that IUC is associated with depression, as well as anxiety (Dar, Iqbal, \& Mushtaq, 2017). Closer examination of the mechanism in previous work reveals that worry acts as a mediator between IUC and symptoms of depression and anxiety (Dar et al., 2017). While depression was included in the model separately for comparison to other works, it should be highlighted that depressive symptoms are considered to be conceptually related to the group membership due to the well-established relationship between depression and OCD (e.g., Pallanti et al., 2011), and attempts at statistical removal of variance would be 
detrimental to the construct. The reader is encouraged it interpret the aforementioned finding with this in mind.

Taken together, results from the subscale of the OBQ suggest specificity of IU beliefs to OCD and HD. This study provides initial evidence that IUC beliefs may not be associated with the GDs. Results for the IUC highlight the complexity and conceptual relatedness among OCRD symptoms, depression, and anxiety. Nevertheless, it also suggests specificity to OCD and HD when depression is included in a shared variance approach to group membership.

When perfectionism was measured using the FMPS, the current study did not find a significant difference in the total perfectionism score between any of the clinical groups and healthy controls. Examination of the subscales revealed that those in the HD group had significantly lower levels of personal standard perfectionism than all other groups. Additionally, those in the OCD group had higher perfectionism on the Control of Mistakes/Doubts subscale than controls. This differing profile of perfectionism highlights the importance of multidimensional measurement of this construct. The null finding of perfectionism in the GD group is somewhat discrepant to previous literature, as past research has indicated an association (e.g., Bottesi et al., 2016; O’Connor et al., 2002; Rehm, 2015). All three clinical groups had moderate symptom severity once GD was broken down into TTM and ED, respectively. Nevertheless, severity may be an important clinical correlates (Abramovitch et al., 2015). Thus, it is possible that a more nuanced relationship exists between perfectionism and the OCRDs that includes symptom severity.

\section{Self-report indecision}

Those in the OCD and HD groups reported significantly more indecision than controls. This is consistent with both the theoretical importance of decision-making in both disorders, and 
also previous research finding self-report indecision in OCD (Frost \& Shows, 1993). Given the dearth of literature examining self-report indecision, this is an important area of future research, particularly given the previous finding that indecision significantly predicts quality-of-life in those with subclinical OCD symptoms (Taillefer, Liu, Ornstein, \& Vickers, 2016).

The majority of research has examined indecision using gambling paradigms. Such paradigms in OCD has been decidedly mixed, with some studies finding impaired performance (Cavedini et al., 2002; Kocak et al., 2010; Viswanath et al., 2009) while an equal number of null findings have emerged (Lawrence et al., 2006; Nielen et al., 2002; Watkins et al., 2005). In HD, the majority of studies using a decision-making paradigm failed to find a significant impairment (Blom et al., 2011; Grisham et al., 2007, 2010;; Tolin \& Villavicencio, 2011), despite self-report indecisiveness (Grisham et al., 2010; Steketee et al., 2003; Tolin \& Villavicencio, 2011; Wincze et al., 2007). Decision-making in the other related disorders has not been well studied.

This highlights the importance of examining decision-making multidimensionally, as it may not be a unitary construct, with aspects of organizational strategies, impulsivity, risk perception, and ambiguity (Dittrich \& Johansen, 2013; Olley et al., 2007; Starcke et al., 2010;

Stern et al., 2013). Additionally, it raises the possibility that dysfunctional beliefs about decisionmaking may exist without an objective deficit (i.e., dysfunctional belief rather than a corticostriatal deficit). Further research should clarify whether this is an objective or subjective deficit, as it has implications for treatment targets. The current study suggests specificity of subjective indecision for OCD and HD.

\section{Summary}

It was predicted that there would be general endophenotypes underlying all of the OCRDs, and that there would be some specific CEs linked to some OCRDs but not others. While 
it should be noted that this work is very preliminary and the current study was significantly underpowered, results of this work largely support the aforementioned hypothesis. Specifically, a review of previous work and integration of the results from the current study suggest preliminary support for inhibitory dysfunction as a shared CE across the spectrum, with verbal memory specific to OCD, and interference control and set-shifting specific to OCD and HD. Due to the conflicting findings in nonverbal memory, trends were difficult to establish. Examination of dysfunction belief CEs found specificity of Responsibility/Threat beliefs and Importance/Control of Thoughts beliefs to OCD. IU and self-report indecision was associated with OCD and HD. Despite a null finding in the current study, past work suggests that perfectionism may be a general CE across the OCRDS; however, this and other studies' null findings highlight the importance of multi-dimensional measurement of perfectionism.

(2) Is self-report impulsivity correlated with the OCRDs?

It was predicated that impulsivity would be associated with the related disorders, but not OCD. Results were partially in support of this hypothesis, as there was no significant association between any of the clinical groups and impulsivity, for either the total score or associated subscales. The lack of a finding between OCD and impulsivity is in keeping with more contemporary conceptualization, suggesting that $\mathrm{OCD}$ is more related to compulsivity than impulsivity (Chamberlain, Leppink, Redden, \& Grant, 2016). The lack of a significant finding in HD adds to previous research finding that age accounted for the relationship between HD symptoms and impulsivity (Rasmussen et al., 2013). The lack of a finding in the GD group is surprising, given previous research using the BIS-11 that found cognitive impulsivity (Adams, 2012; Hayes et al., 2009) and motor impulsivity (Hayes et al., 2009) predicted symptom severity. However, a closer examination of symptom severity in the GD group revealed moderate levels of 
symptom severity, as aforementioned. Taken together, self-report impulsivity in the GDs may be a function of symptom severity.

(3) Is there an association between candidate endophenotypes and QOL?

Examination of overall QOL revealed that those in the OCD and HD group had significantly impaired QOL compared to controls. When QOL was broken down by domain, all clinical groups reported impaired social, family life, and home responsibilities compared to controls. Interestingly, only the OCD group reported significantly more impairment in work/school than controls. This is consistent with previous literature, which found that $77.2 \%$ of individuals with OCD indicated that their ability to work was impeded (Sorensen et al., 2004). However, the null finding in HD is surprising, given that previous research has found greater work disability (Diefenbach et al., 2013; Landau et al., 2011; Pertusa et al., 2008) than controls. Examination of the items on the scale reveals that it is possible that those in the HD group attributed their unemployment to symptoms of another co-morbid disorder (e.g., depression). The finding of impaired social functioning in the GDs supports previous research indicating that social and interpersonal functioning are particularly affected in TTM and ED, with deficits in the moderate to severe range (Flessner et al., 2009; Odlaug \& Grant, 2008a).

Examination of the relationship between neurocognitive measures and QOL revealed results in support of the general hypotheses that some CEs would be associated with QOL. Specifically, variables on the WCST, the TMT, the RCFT, and the digit symbol were significantly correlated with QOL. This suggests that interference control, cognitive flexibility, and nonverbal memory may be areas that impact day to day functioning more explicitly. Examination of dysfunctional belief CEs and QOL revealed significant correlations between the IUC, IS, and BIS with both the QLES and the SDS. Examination of the relationship between 
neurocognitive measures and dysfunctional beliefs revealed that variables of the WCST and the copy variable on the RCFT were significantly correlated with perfectionism. This is consistent with the literature suggesting that perfectionism influences measures of executive functioning (e.g., Slade et al., 2009).

\section{Limitations}

As aforementioned, this dissertation had significant limitations. First and foremost, the small sample size and corresponding lack of power increased the chance of Type II error (failing to detect an effect) and likely contributed to the relative absence of positive findings. Thus, nonsignificance could mean either; 1) that there is no effect; or 2) that the study was too underpowered to detect significant group differences, particularly for small effects. As demonstrated by the power analysis, the current study was grossly underpowered, particularly for the analyses where there was missing data. While null findings can dissuade researchers from pursuing lines of inquiry, it is hoped that this work will serve as pilot work and highlight areas for improved research design and generate areas for future research.

The current study also had an inflated Type 1 error rate due to multiple comparisons (as noted, the study-wide Type 1 error rate was between $96 \%$ and 100\%). The need to correct for multiple comparisons in this area has been highlighted in reviews of the neuropsychological literature in OCD, where power tends to be low, sample size is relatively small, and there are numerous dependent variables (Abramovitch et al., 2015; Kuelz et al., 2004). This study used the Holm procedure (Holm, 1979) in an attempt to mitigate the influence of multiple comparisons while maximizing limited power. However, the use of the Holm's procedure rather than the use of an overall conservative significance threshold does increase Type 1 error. 
A further statistical challenge was handling pre-existing group difference due to the obvious inability for random group assignment (i.e, diagnostic status is not random group assignment). There is no theoretically sound or agreed upon method to statistically control for real group differences (see Miller \& Chapman, 2001). As aforementioned, it could be argued that gender and age, and particularly depressive symptoms, are defining group characteristics and, as such, removing the variance associated with these constructs may be detrimental to the construct. Although presenting with its own limitations, one recommendation to statistically manage real group differences is to include the related variable in the model. Thus, this study used hierarchical regression. However, due to sample size limitations, interactions were not included in the model.

Additionally, the full spectrum of the OCRDs was not captured. The GD group was composed of both TTM and ED. Although there is some indication that these disorders more closely share phenomenology than do the other OCRDs (e.g., Lochner et al., 2002; Odlaug \& Grant, 2008a/b) and there is precedent for combining the two groups for statistical analysis (e.g., Flessner et al., 2009; Grant \& Christenson, 2007; Hanna et al., 2005), there has also been some, albeit limited, indication of differing neurocognitive performance between the two disorders (e.g., Grant et al., 2011).Thus, if differing neurocognitive deficits exist between TTM and ED, they may not have been detected when the sample was collapsed. Additionally, due to difficulties recruiting a BDD sample due to low rates of treatment seeking behaviour (Conroy et al., 2008), this study did not include a BDD group, and therefore was missing one of the OCRDs as outlined in DSM-5, limiting comparison within the spectrum. As well, this study was conducted prior to the release of the SCID-5 (2016). Thus, available clinical interviews did not assess the OCRDs. 
Inter-rater reliability statistics were not computed for the insert and this is a limitation of the methodology

Moreover, sample characteristics (representativeness) may influence the generalizability of the results. The present study largely recruited from The Frederick W. Thompson Anxiety Disorders Centre at Sunnybrook Health Sciences Centre, an outpatient, specialty, clinic that requires a referral from a family physician or psychiatrist. This raises many issues including differing clinical characteristics (e.g., severity, chronicity, comorbidities), socio-economic status, and the fact that the sample was treatment-seeking. Thus, it is likely that this sample has differing characteristics than other samples recruited from differing sources (e.g., general outpatient clinics, community recruitment). There has been some indication that specialty clinic samples often present with more severe and complex clinical presentations (McConaughy \& Achenbach, 1994). Additionally, there has been some research to suggest that those with higher socioeconomic status are referred more often to specialists (Sørensen, Olsen, \& Vedsted, 2009). When speaking about sample representativeness, it is also important to note that this study is largely made up of WEIRD participants, an acronym to describe participants that are Western, educated, and from industrialized, rich, and democratic countries (Henrich, Heine, \& Norenzajan, 2010). Thus, a cautionary note should be made about the representative nature of participants in the current study.

Similarly, those in the OCD group reported a mean of four years of post-secondary education (16 years total), equivalent to an undergraduate degree. While this was not a statistically significant difference between the groups, it may be clinically significant. The mean education level of all three clinical groups in the current study was higher than that of the nonpsychiatric controls. This is surprising, given recent research finding that individuals with OCD 
were more likely to fail courses and less likely to achieve a postgraduate degree (Pérez-Vigil et al., 2018). However there are some important sample differences between the sample of PérezVigil et al. (2018) and the sample of the current dissertation. Namely, the Pérez-Vigil et al. (2018) sample used a Swedish, population-based sample, and Sweden is one of the Scandinavian countries where tuition in postsecondary is state covered. Thus, it is possible that socioeconomic status in Canada, where post-secondary tuition is not state covered, has a mediating/moderating effect; particularly given the current sample was recruited from a specialty clinic, and research suggests a selection bias such that that those with higher socioeconomic status are referred more often to specialists (Sørensen, Olsen, \& Vedsted, 2009). Furthermore, the results of Pérez-Vigil et al. (2018) were strongest for early-onset OCD, while the current sample was heterogeneous in terms of age of onset. With those presenting with a late age of onset, it is feasible that an undergraduate degree was completed prior to illness onset. In previous research, the range in education level across studies has been vast, for example 10.3 years (Kitis et al., 2007) to 17.1 (Exner et al., 2009) years, perhaps highlighting a more nuanced relationship between an OCD diagnosis and education attainment.

Given the range in education level between studies, comparing across studies is difficult, as it is well established that education affects scores on most neuropsychological tests (Lezak et al., 2012). Thus, "it is reasonable to assume a major baseline difference in neuropsychological performance" (Abramovitch et al., 2015, p. 116) based on years of education. Nevertheless, the education attainment by the current sample does highlight issues with generalizability and sample representativeness and suggests that this sample may not be entirely representative of community samples. 
Additionally, factors such as medication status or past psychotherapy were not controlled. Participants did have to be on a stable medication regime for six weeks prior to study inclusion, but some participants were medicated while others were medication free, and there was variance in the class of medications used (e.g., SSRI vs. Tricyclic vs SNRI). While there is indication that SSRIs do not impact neuropsychological performance (Mataix-Cols, Alonso, Pifarre, Menchon, \& Vallejo, 2002; Paul, Gray, Love, \& Lange, 2007), there is some evidence that the tricyclic antidepressant clomipramine (Allen, Curran, \& Lader, 1991; Serretti et al., 2010) and also neuroleptic medication (Veselinovic et al., 2013) may influence performance. In the current study, only two participants were taking clomipramine and no participants were augmenting with antipsychotics (neuroleptics). Thus, it is unlikely that these identified medications influenced neurocognitive results. Nevertheless, it is possible that current, or past, medication status could have impacted findings for the associated constructs. Similarly, past psychotherapy trials varied between participants, with some participants self-reporting rounds of evidence-based psychotherapy while others were naïve to psychotherapy treatment. One study to date in the OCRDs found that spatial working memory improved following CBT (Nedeljkovic, Kyrios, Moulding, \& Doron, 2011). Thus, it is possible that for those who had received historical psychotherapy, any CEs (either neurocognitive or belief-related) may have improved prior to study participation.

Similarly, due to the nature of the sample, individuals with co-morbidities were included in the study, including other co-morbid OCRDs. While this lack of control is reminiscent of clinical practice and, to a larger extent, has improved external validity and generalizability, it does influence internal validity. Although it was not possible to 'control' for symptoms of all comorbid conditions, this study did include depressive symptoms in the model (see above in 
regards to difficulties accounting for real group differences), as depressive symptoms have been found to impact neuropsychological performance in OCD (Basso et al., 2001; Moritz et al., 2001). There was no statistically significant difference between clinical groups on measures of depressive symptoms. However, there was a high rate of depression noted in the sample overall.

Additionally, it is well established that OCD is a heterogeneous condition. Factor analyses have identified four or five different symptom subtype clusters (Bloch et al., 2008; Cullen et al., 2006; Mataix-Cols et al., 2004; Stewart et al., 2008; Summerfeldt, Richter, Antony, \& Swinson, 1999). The four factor solutions typically include:

aggressive/sexual/religious/somatic obsessions/and checking rituals; symmetry obsessions with ordering/counting/repeating compulsions; contamination/and cleaning rituals; and, historically, hoarding (Stewart et al., 2008). Heterogeneity has also been established in terms of age-of-onset, with research indicating a bimodal distribution of onset; early onset (mean onset 11 years) and late onset (mean onset 23 years), that appear to differ in terms of gender distribution, severity, comorbidity, etc. (see Taylor, 2011 for a review). Heterogeneity is also observed with comorbidity, particularly with tics (Conelea et al., 2014; Hirschtritt, Bloch, \& Mathews, 2017). Evidence suggests that these subtypes may be etiologically distinct (e.g., Katerberg et al., 2010; Leckman et al., 2003; Mataix-Cols et al., 2004). As aforementioned, this heterogeneity may account for replication challenges in the neurocognitive literature, as there has been some suggestion of differing neurocognitive profiles based on subtype (Nedeljkovic et al., 2009; McGuire et al., 2014). While the recommendation has been to include subtyping in analyses, the current study was not able to include subtypes due to sample size restrictions, but this remains an important future avenue for research. 
Another limitation of the current study is that symptom severity was not included in statistical models. While all of the clinical groups reported moderate symptom severity, one important recommendation from the neurocognitive literature has been to include clinical correlates (more specifically symptom severity) in analyses, as severity may be associated with outcome (Abramovitch et al., 2015). Due to aforementioned significant power limitations, severity was not a variable included in analysis. This is a limitation to the current work, and the results should be interpreted with this in mind.

\section{Broader Implications}

With the recent reclassification of OCD and related disorders into their own spectrum of disorders in DSM-5 (American Psychiatric Association, 2013), there has been increased emphasis on the differing and shared phenomenology and neurobiology of these disorders; particularly the differentiation of HD as its own disorder and not merely a symptom of OCD. The inclusion of these disorders in the same class suggests a belief that these disorders share etiological factors. The need to identify broad versus specific etiological factors has been highlighted (Taylor, 2012). Results of the current study, and a synthesis of previous literature, suggest some CEs are broad while others are likely disorder-specific. The identification of broad versus specific CEs have implications for diagnostic classification, etiology, course, and treatment (e.g., etiological-based diagnostic classification vs. symptom-based classification, general vs. specific psychotherapy treatment targets).

With the expanded conceptualization of endophenotypes as a vulnerability factor (e.g., Taylor, 2012), the mediating variable model suggests that intervening to reduce the level of the endophenotype could lower the risk of psychopathology (Kendler \& Neale, 2010). For example, if perfectionistic beliefs were found to be a reliable $\mathrm{CE}$, reducing perfectionistic beliefs through 
cognitive restructuring may lower the risk of developing psychopathology or may allow clinicians to intervene early to alter the course of psychopathology. Another example could be cognitive remediation to rehabilitate neurocognitive deficits, particularly with preliminary research suggesting improved efficacy of behaviour therapy in HD with adjunctive cognitive remediation (Ayers et al., 2014). Thus, the identification of CEs could identify risk factors of psychopathology and contribute to prevention or early intervention work, in addition to highlighting novel treatment options.

As aforementioned, this study included CEs from two theoretical perspectives; the corticostriatal-thalamic model (see Menzies et al., 2008 for a review) and the learning model (Clark, 2004; Frost \& Steketee, 2002; Rachman, 1997). The need for a comprehensive approach has been highlighted in behavioural-genetic work, as one model alone is insufficient to a comprehensive model of etiology (e.g., Taylor, 2011). That noted, there are other major etiological models not addressed in the current study; the focus in the current work was specifically on neurocognitive and dysfunctional beliefs as these have been suggested to be amongst the most robust endophenotypes, would be more readily translatable to clinical use, and have not been explored broadly across the OCRDs.

\section{Future Directions}

Recommendations for future research include continued evaluation of identified CEs from both theoretical perspectives to determine the specificity of the identified CEs. There also remains a need to identify novel CEs to better understand complex disorders (Taylor, 2012), particularly in the GDs and BDD as research examining CEs in these disorders is in its infancy. As many of the identified effect sizes, particularly for neurocognitive findings, are small to moderate, future work should continue to prioritize sample size and corresponding power. The 
nature of this work and chronic difficulties with power highlights the need for collaboration between institutions and the importance of meta-analyses.

Importantly, it is widely accepted that OCD is a heterogeneous condition (e.g., Katerberg et al., 2010; Leckman et al., 2003; Mataix-Cols et al., 2004); however, most studies, including the current study, treat it homogeneously. This likely contributes to discrepancies in current literature and difficulties with replication. With the findings that CEs may vary with differing patterns in neurocognition (e.g., Dittrich et al., 2011; Nedelikovic et al. 2009), differing dysfunctional beliefs (Taylor et al., 2010), and differential responses to treatment (e.g., Alarcon, Libb \& Spitler, 1993; Ravizza, Barzega, Bellino, Bogetto, \& Maina, 1995; Shetti et al., 2005); future research should examine CEs within etiologically-based subtypes.

Further examination of the relationship between CEs and intervention (both pharmacological and psychotherapy) is also recommended, as research examining this relationship is in its infancy. For example, important research questions include whether CEs change and are malleable with treatment, whether early intervention/prevention with identified CEs reduces psychopathology, and whether CEs predict treatment response. To date, examination of neurocognition CEs before and after treatment has only been scrutinized in a very limited number of studies. Preliminary research suggests that pharmacotherapy (e.g., fluoxetine in OCD; Neilen \& den Boer, 2003) did not alter neurocognitive impairments, with results showing enduring neurocognitive impairment following symptom improvement. Similarly, only one study to date in the OCRDs examined how neurocognitive deficits are affected by psychotherapy. This study found that spatial working memory improved following CBT (Nedeljkovic et al., 2011). This shows promising results regarding the potential malleability of neurocognitive CEs with psychotherapy. Additionally, neurocognitive CEs as a predictor of 
treatment response is a novel and important avenue of research. One study to date has examined neurocognition as a predictor of response to pharmacotherapy (e.g., lamotrigine; Grant et al., 2011), finding that set-shifting ability predicted treatment response, with treatment responders demonstrating impaired cognitive flexibility at baseline. Of note in the aforementioned study, lamotrigine was not found to be significantly efficacious compared to placebo control when the entire sample was analyzed. Similarly, examination of the relationship between dysfunctional belief CEs and intervention have found that changes in obsessive/maladaptive beliefs predicted symptom severity at post-treatment in both inpatient (Diedrich et al., 2016) and outpatient (Wilhem et al., 2015) receiving CBT.

Future studies should examine cognitive remediation as a novel treatment target. As noted, CBT response rates (measured according to clinically significant change, a conservative estimate of high end state function and reliable change) in the OCRDs range from $63 \%$ of OCD treatment completers (Abramowitz et al., 2003) to $41 \%$ of hoarding completers (Steketee et al., 2010), which is considered relatively good. However, 37\%-59\% of patients are partial or nonresponders, indicating that additional areas of intervention or ways to optimize current treatment are necessary. Although research into cognitive remediation in OCRDs is in its infancy, preliminary research in HD reveals promising results (DiMauro et al., 2014), particularly as an adjunct to CBT; patients with HD showed significant reductions in hoarding severity, with a doubling of previously reported response rates, suggesting that cognitive rehabilitation with exposure therapy is a promising approach to treatment (Ayers et al., 2014). This research parallels literature in other psychiatric disorders, particularly schizophrenia, suggesting cognitive remediation may be an important treatment target (see Kurtz et al., 2001 for a review). 


\section{Conclusions}

The current dissertation examined candidate endophenotypes (CEs) across the obsessive compulsive spectrum from both primary theoretical models to elucidate general versus specific CEs. The current dissertation also examined several CEs multidimensionally, examined several novel potential CEs (indecision and impulsivity), and examined the relationship between CEs and of QOL. The results of the current research highlight the discrepancy of findings in the field, but do suggest differing CEs profiles across the spectrum. Of note, those with HD performed worse than controls on measures of response inhibition and set-shifting. Examination of dysfunctional belief CEs revealed specificity of Responsibility/Threat beliefs and Importance/Control of Thoughts beliefs to OCD. Perfectionism/IU appear to be broad CEs; however, differing specificity was revealed depending on the measure utilized to measure the construct. Examination of self-report indecision revealed specificity to OCD and HD. However, the current study had significant limitations, including insufficient power to detect small effect sizes. Thus, null findings should be interpreted with caution. Future research should continue to examine identified and novel CEs to determine the specificity of these CEs, should examine etiologically-based subtypes of OCD, and should continue to examine the relationship between CEs and intervention. A better understanding of CEs could contribute to a better understanding of these complex disorders (Taylor, 2012). 


\section{Reference List}

Abbruzzese, M., Bellodi, L., Ferri, S., \& Scarone, S. (1995). Frontal lobe dysfunction in schizophrenia and obsessive-compulsive disorder: A neuropsychological study. Brain and Cognition, 27, 202-212.

Abbruzzese, M., Ferri, S., \& Scarone, S. (1995). Wisconsin card sorting test-performance in obsessive-compulsive disorder-no evidence for involvement of dorsolateral prefrontal cortex. Psychiatry Research, 58, 37-43.

Abbruzzese, M., Ferri, S., \& Scarone, S. (1997). The selective breakdown of frontal functions in patients with obsessive-compulsive disorder and in patients with schizophrenia: A double dissociation experimental finding. Neuropsychologia, 35, 907-912.

Abraham, K. (1921). Contributions to the theory of the anal character. Selected papers, 370-392. Abramovitch, A., Abramowitz, J.S., \& Mittelman, A. (2013). The neuropsychology of adult obsessive- compulsive disorder: A meta-analysis. Clinical Psychological Review, 33, 1163-1171.

Abramovitch, A., \& McKay, D. (2016). Behavioral impulsivity in obsessive-compulsive disorder. Journal of Behavioral Addictions, 5(3), 395-397.

Abramovitch, A., Mittelman, A., Tankersley, A.P., Abramowitz, J.S., \& Schweiger, A. (2015). Neuropsychological investigations in obsessive-compulsive disorder: A systematic review of methodological challenges. Psychiatry Research, 228, 112-120.

Abramowitz, J.S., \& Deacon, B.J. (2006). Psychometric properties and construct validity of the Obsessive-Compulsive Inventory-Revised: Replication and extension with a clinical sample. Journal of Anxiety Disorders, 20, 1016-1035. 
Abramowitz, J. S., Franklin, M. E., Schwartz, S. A., \& Furr, J. M. (2003). Symptom presentation and outcome of cognitive-behavior therapy for obsessive-compulsive disorder. Journal of Consulting and Clinical Psychology, 71, 1049-1057.

Abramowitz, J. S., Lackey, G. R., \& Wheaton, M. G. (2009). Obsessive-compulsive symptoms: the contribution of obsessional beliefs and experiential avoidance. Journal of Anxiety Disorders, 23, 160-166.

Adams, T. G. (2012). The unique roles of affect and impulsivity in the prediction of skin picking severity. Journal of Obsessive-Compulsive and Related Disorders, 1, 138-143.

Alarcon, R. D., Libb, J. W., \& Spitler, D. A. (1993). A predictive study of obsessive compulsive disorder response to clomipramine. Journal of Clinical Psychopharmacology, 13, 210-213.

Alexander, E. G., Crutcher, M. D., \& DeLong, M. R. (1990). Basal ganglia-thalamocortical circuits: parallel substrates for motor, oculomotor, 'prefrontal' and 'limbic' functions. Progress in Brain Research, 85, 119-146.

Alexander, E. G., DeLong, M. R., \& Strick, P. L. (1986). Parallel organization of functionally segregated circuits linking basal ganglia and cortex. Annual Review of Neuroscience. 9, 357-381.

Allen, D., Curran, H.V., \& Lader, M. (1991). The effects of repeated doses of clomipramine and alprazolam on physiological, psychomotor and cognitive functions in normal subjects. European Journal of Clinical Pharmacology, 40, 355-362.

American Psychiatric Association. (2000). Diagnostic and statistical manual of mental disorders ( $4^{\text {th }}$ ed.-text revision). Washington, DC: Author.

American Psychiatric Association. (2006). American Psychiatric Association practice guidelines for the treatment of psychiatric disorders: Compendium. Washington, DC: Author. 
American Psychiatric Association. (2007). Practice Guideline for the treatment of patients with obsessive-compulsive disorder. Arlington, VA: Author.

American Psychiatric Association. (2013). Diagnostic and statistical manual of mental disorders: DSM-5 (5th ed.). Arlington, VA: Author.

Aouizerate, B., Guehl, D., Cuny, E., Rougier, A., Bioulac, B., Tignol, J., et al. (2004).

Pathophysiology of obsessive-compulsive disorder: a necessary link between phenomenology, neuropsychology, imagery and physiology. Progressive Neurobiology, $72,195-221$.

Army Individual Test Battery. (1944). Manual of directions and scoring. Washington, DC: War Department, Adjutant General's Office.

Arnold, P., Askland, K., Barlassina, C., Bellodi, L., Bienvenu, O., Black, D.,...Zai, G. (2017). Revealing the complex genetic architecture of obsessive-compulsive disorder using meta-analysis. Molecular Psychiatry, 23(5), 1181-1188.

Arnold, L. M., McElroy, S. I., Mutasim, D. F., Dwight, M. M., Lamerson, C. I., \& Morris, E. M. (1998). Characteristics of 34 adults with psychogenic excoriation. Journal of Clinical Psychiatry, 59, 509-514.

Arnold, P.D., \& Richter, M.A. (2007). Genetics of obsessive-compulsive disorder: Evidence from pediatric and adult studies. In E.A. Storch, G.R. Geffken, \& T. Murphy (Eds.), Handbook of child and adolescent obsessive-compulsive disorder (pp.175-212). Mahwah, NJ: Erlbaum.

Aron, A. R., Fletcher, P. C., Bullmore, E. T., Sahakian, B. J., \& Robbins, T. W. (2003). Stopsignal inhibition disrupted by damage to right inferior frontal gyrus in humans. Natural Neuroscience, 6, 115-116.

Ayers, C. R., Saxena, S., Espejo, E., Twamley, E. W., Granholm, E., \& Wetherell, J. L. (2014). 
Novel treatment for geriatric hoarding disorder: an open trial of cognitive rehabilitation paired with behavior therapy. American Journal of Geriatric Psychiatry, 22(3), 248-252.

Ayers, C.R., Saxena, S., Golshan, S., \& Wetherell, J.L. (2010). Age at onset and clinical features of late life compulsive hoarding. International Journal of Geriatric Psychiatry, 25. 142149.

Ayers, C. R., Wetherell, J. L., Schiehser, D., Almklov, E., Golshan, S., \& Saxena, S. (2013). Executive functioning in older adults with hoarding disorder. International Journal of Geriatric Psychiatry, 28(11), 1175-1181.

Bailey, M. L. (2002). Neuropsychological Indicators in Body Dysmorphic Disorder. Unpublished doctoral dissertation, New School for Social Research, New York.

Bannon, S., Gonsalvez, C. J., Croft, R. J., \& Boyce, P. M. (2002). Response inhibition deficits in obsessive-compulsive disorder. Psychiatry Research, 110(2), 165-174.

Barlow, D.H., Sauer-Zavala, S., \& Ellard, K.K. (2010). Unified protocol for transdiagnostic treatment of emotional disorders. London: Oxford University Press.

Barnett, R., Maruff, P., Purcell, P., Wainwright, K., Kyrios, M., Brewer, W., et al. (1999). Impairment of olfactory identification in obsessive-compulsive disorder. Psychological Medicine, 29, 1227-1233.

Barr, L., Goodman, W., \& Price, L. (1992). Acute exacerbation of body dysmorphic disorder during tryptophan depletion. The American Journal of Psychiatry, 149, 1406-1407.

Bartz, J.A., \& Hollander, E. (2006). Is obsessive-compulsive disorder an anxiety disorder? Progress in Neuro-Psychopharmacology and Biological Psychiatry, 30, 228-238.

Basso, M.R., Bornstein, R.A., Carona, F., \& Morton, R. (2001). Depression accounts for 
executive function deficits in obsessive-compulsive disorder. Neuropsychiatry, Neuropsychology, and Behavioral Neurology, 14, 241-245.

Beck, A. T., Epstein, N., Brown, G., \& Steer, R. A. (1988). An inventory for measuring clinical anxiety: psychometric properties. Journal of Consulting and Clinical Psychology, 56(6), 893-897.

Beck, A. T., Steer, R. A., \& Brown, G. K. (1996). Manual for the Beck Depression Inventory-II. San Antonio, TX: The Psychological Corporation.

Bedard, M.J., Joyal, C.C., Godbout, L., \& Chantal, S. (2009). Executive functions and obsessivecompulsive disorder on the importance of subclinical symptoms and other concomitant factors. Archives of Clinical Neuropsychology, 24, 585-598.

Benton, A.L. (1974). Revised Visual Retention Test ( $4^{\text {th }}$ ed.). San Antonio, TX: The Psychological Corporation.

Berg, E. (1948). A simple objective technique for measuring flexibility in thinking. Journal of General Psychology, 39, 15-22.

Bloch, M. R., Elliott, M., Thompson, H., \& Koran, L. M. (2001). Fluoxetine in pathologic skinpicking: open label and double-blind results. Psychosomatics, 42, 314-319.

Bloch, M. H., Landeros-Weisenberger, A., Kelmendi, B., Coric, V., Bracken, M. B., \& Leckman, J. F. (2006). A systematic review: antipsychotic augmentation with treatment refractory obsessive-compulsive disorder. Molecular Psychiatry, 11(7), 622-32.

Bloch, M.H., Landeros-Weisenberger, A., Rosario, M.C., Pittenger, C., \& Leckman, J.F. (2008). Meta-analysis of the symptom structure of obsessive-compulsive disorder. American Journal of Psychiatry, 165, 1532-1542.

Blom, R.M., Samuels, J.F., Grados, M.A., Chen, Y., Bienvenu, O.J., Riddle, M.A., et al. (2011). 
Cognitive functioning in compulsive hoarding. Journal of Anxiety Disorders, 25(8), $1139-1144$.

Boelen, J.F., \& Reijntjes, A. (2009). Intolerance of uncertainty and social anxiety. Journal of Anxiety Disorders, 23(1), 130-135.

Bohne, A., Savage, C.R., Deckersbach, T., Keuthen, N.J., Jenike, A., Tuschen-Caffier, B., et al. (2005). Visuospatial abilities, memory, and executive functioning in trichotillomania and obsessive-compulsive disorder. Journal of Clinical Experimental Neuropsychology, 27(4), 385-399.

Boisseau, C.L., Thompson-Brenner, H., Pratt, E.M., Farchione, T.J., \& Barlow, D.H. (2013). The relationship between decision-making and perfectionism in obsessive-compulsive disorder and eating disorders. Journal of Behavioural Therapy in Experimental Psychiatry, 44, 316-321.

Bolton, D., Rijsdijk, F., O’Connor, T.G., Perrin, S., \& Eley, T.C. (2007), Obsessive-compulsive disorder, tics and anxiety in 6-year-old twins. Psychological Medicine, 37, 39- 48.

Boone, K. B., Ananth, J., Philpott, L., Kaur, A., \& Djenderedjian, A. (1991). Neuropsychological characteristics of non-depressed adults with obsessive-compulsive disorder. Neuropsychiatry, Neuropsychology and Behavioral Neurology, 4, 96-109.

Boswell, J.F., Thompson-Hollands, J., Farchione, T.J., \& Barlow, D.H. (2013). Intolerance of uncertainty: A common factor in the treatment of emotional disorders. Journal of Clinical Psychology, 69(6), 32-47.

Bottesi, G., Ghisi, M., Carraro, E., Barclay, N., Payne, R., \& Freeston, M.H. (2016). Revising the intolerance of uncertainty model of generalized anxiety disorder: Evidence from UK and Italian undergraduate samples. Frontiers in Psychology, 7, 1723. 
Bowie, C. R., Reichenberg, A., Patterson, T., Heaton, R. K., \& Harvey, P. (2006). Determinants of real-world functional performance in schizophrenia subjects: correlations with cognition, functional capacity, and symptoms. The American Journal of Psychiatry, 163(3), 418-425.

Bradbury, C., Cassin, S. E., \& Rector, N. A. (2011). Obsessive beliefs and neurocognitive flexibility in obsessive-compulsive disorder. Psychiatry Research, 187, 160-165.

Brakoulias, V., Eslick, G. D., \& Starcevic, V. (2015). A meta-analysis of the response of pathological hoarding to pharmacotherapy, Psychiatry Research, 229, 272-276.

Brakoulias, V., Starcevic, V., Sammut, P., Berle, D., Milicevic, D., Moses, K., et al. (2011). Obsessive-compulsive spectrum disorders: a comorbidity and family history perspective. Australasian Psychiatry, 19(2), 151-155.

Buhlmann, U., Cook, L.M., Fama, J.M., \& Wilhelm, S. (2007). Perceived teasing experiences in body dysmorphic disorder. Body Image, 4(4), 381-385.

Buhlmann, U., Etcoff, N.L., \& Wilhelm, S. (2008). Facial attractiveness ratings and perfectionism in body dysmorphic disorder and obsessive-compulsive disorder. Journal of Anxiety Disorders, 22, 540-547.

Buhlmann, U., Glaesmer, H., Mewes, R., Fama, J. M., Wilhelm, S., Brahler, E., et al. (2010). Updates on the prevalence of body dysmorphic disorder: a population-based survey. Psychiatry Research, 178(1), 171-175.

Buhr, K., \& Dugas, M. J. (2002). The intolerance of uncertainty scale: psychometric properties of the English version. Behavior Research and Therapy, 40, 931-945. 
Burdick, K.E., Robinson, D.G., Malhotra, A.K., \& Szeszko, P.R. (2008). Neurocognitive profile analysis in obsessive-compulsive disorder. Journal of the International Neuropsychological Society, 14(4), 640-645.

Cabrera, A. R., McNally, R. J., \& Savage, C. (2001). Missing the forest for the trees? Deficient memory for linguistic gist in obsessive-compulsive disorder. Psychological Medicine, 31, 1089-1094.

Cambridge Cognition Ltd. (2006). Cambridge Neuropsychological Test Automated Battery (CANTAB). Cambridge, United Kingdom: Tunsbridge Court.

Cannon, T. D., Gasperoni, T. L., van Erp, T. G., \& Rosso, I. M. (2001). Quantitative neural indicators of liability to schizophrenia: implications for molecular genetic studies. American Journal of Medical Genetics, 105, 16-19.

Cannon, T. D., \& Keller, M. C. (2006). Endophenotypes in the genetic analyses of mental disorders. Annual Review of Clinical Psychology, 2, 267-290.

Cardinal, R. N., \& Aitken, M. R. F. (2006). ANOVA for the Behavioural Sciences Researcher. Mahwah, NJ: Erlbaum.

Carey, C., \& Gottesman, I. (1981). Twin and family studies of anxiety, phobic and obsessive disorders. In D. Klein, \& J. Rabkin (Eds.), Anxiety: New research and changing concepts. New York: Raven Press.

Cath, D.C., Nizar, K., Boomsma, D., \& Mahews, C.A. (2017). Age-specific prevalence of hoarding and obsessive compulsive disorder: A population-based study. American Journal of Geriatric Psychiatry, 25 (3), 245-255.

Cavendini, P., Gorini, A., \& Bellodi, L. (2006). Understanding obsessive-compulsive disorder: focus on decision making. Neuropsychology Review, 16, 3-15. 
Cavedini, P., Riboldi, G., D'Annucci, A., Belotti, P., Cisima, M., \& Bellodi, L. (2002). Decisionmaking heterogeneity in obsessive-compulsive disorder: ventromedial prefrontal cortex function predicts different treatment outcomes. Neuropsychologia, 40, 205-211.

Cavedini, P., Zorzi, C., Piccinni, M., Cavallini, M. C., \& Bellodi, L. (2010). Executive dysfunctions in obsessive-compulsive patients and unaffected relatives: searching for a new intermediate phenotype. Biological Psychiatry, 67, 1178-1184.

Chakraborty, V., Cherian, A.V., Math, S.B., Venkatasubramanian, G., Thennarasu, K., MataixCols D., et al. (2012). Clinically significant hoarding in obsessive compulsive disorder: results from an Indian study. Comprehensive Psychiatry, 53(8), 1153-1160.

Chamberlain, S. R., Blackwell, A. D., Fineberg, T. W., Robbins, T. W., \& Sahakain, B. J. (2005). The neuropsychology of obsessive compulsive disorder: the importance of failures in cognitive and behavioural inhibition as candidate endophenotypic markers. Neuroscience and Biobehavioral Reviews, 29, 399-419.

Chamberlain, S. R., Fineberg, N. A., Blackwell, A.D., Clark, L., Robbins, T. W., \& Sahakian, B. J. (2007). A neuropsychological comparison of obsessive-compulsive disorder in trichotillomania. Neuropsychologia, 45, 654-662.

Chamberlain, S. R., Fineberg, N. A., Blackwell, A. D., Robbins, T. W., \& Sahakian, B. J. (2006). Motor inhibition and cognitive flexibility in obsessive-compulsive disorder and trichotillomania. American Journal of Psychiatry, 163, 1282-1284.

Chamberlain, S. R., Fineberg, N. A., Menzies, L. A., Blackwell, A. D., Bullmore, E. T., Robbins, T. W., et al. (2007). Impaired cognitive flexibility and motor inhibition in unaffected first-degree relatives of patients with obsessive-compulsive disorder. American Journal of Psychiatry, 164, 335-338. 
Chamberlain, S., Leppink, E.W., Redden, S.A., \& Grant, J.E. (2016). Are obsessivecompulsive symptoms impulsive, compulsive, or both? Comprehensive Psychiatry, 68, 111-118.

Chamberlain, S. R., Menzies, L., Hampshire, A., Suckling, J., Fineberg, N. A., del Campo, N., et al. (2008). Orbitofrontal dysfunction in patients with obsessive-compulsive disorder and their unaffected relatives. Science, 321, 421-422.

Chambers, C. D., Garavan, H., \& Bellgrove, M. A. (2009). Insights into the neural basis of response inhibition from cognitive and clinical neuroscience. Neuroscience Biobehavioral Reviews. 33, 631-646.

Christensen, D. D., \& Greist, J. H. (2001). The challenge of obsessive-compulsive disorder hoarding. Primary Psychiatry, 8, 79-86.

Christenson, G. A., Pyle, R. L., \& Mitchell, J. E. (1991). Estimated lifetime prevalence of trichotillomania in college students. Journal of Clinical Psychiatry, 52, 415-417.

Christensen, K.J., Kim, S.W., Dysken, M.W., \& Hoover, K.M., (1992). Neuropsychological performance in obsessive-compulsive disorder. Biological Psychiatry, 31, 4-18.

Clark, D. A. (2004). Cognitive-Behavioural Therapy for OCD. New York, NY: Guilford Press.

Coetzer, R., \& Stein, D. J. (1999). Neuropsychological measures in women with obsessivecompulsive disorder and trichotillomania. Psychiatry and Clinical Neuroscience, 53(3), 413415.

Cohen, J. (1962). The statistical power of abnormal-social psychological research: A review. The Journal of Abnormal and Social Psychology, 65(3), 145-153.

Cohen, J. (1977). Statistical power analysis for the behavioral sciences. New York: Routledge. Cohen, J., \& Cohen, P. (1983). Applied multiple regression/correlation analysis for the 
behavioral sciences. Hillsdale, NJ: Erlbaum.

Cohen, L. J., Hollander, E., DeCaria, C. M., Stein, D. J., Simeon, D., Liebowitz, M. R., et al. (1996). Specificity of neuropsychological impairment in obsessive-compulsive disorder: a comparison with social phobic and normal control subjects. Journal of Neuropsychiatry and Clinical Neurosciences, 8, 82-85.

Conelea, C.A., Walther, M.R., Freeman, J.B., Garcia, A.M., Sapyta, J., Khanna, M., et al. (2014) Tic-related obsessive-compulsive disorder (OCD): phenomenology and treatment outcome in the pediatric OCD treatment study II. Journal of the American Academy of Child and Adolescent Psychiatry, 53(12), 1308-1316.

Congdon, E., \& Canli, T. (2005). The endopenotype of impulsivity: reaching consilience through behavioral, genetic, and neuroimaging approaches. Behavioral and Cognitive Neuroscience Reviews, 4(4), 262-281.

Conroy, M., Menard, W., Fleming-Ives, K., Modha, P., Cerullo, H., \& Phillips, K. A. (2008). Prevalence and clinical characteristics of body dysmorphic disorder in an adult inpatient setting. General Hospital Psychiatry, 30(1), 67-72.

Cullen, B., Brown, C.H., Riddle, M.A., Grados, M., Bienvenu, J., Hoehn-Saric, R., et al. (2006). Factor analysis of the Yale-Brown Obsessive Compulsive Scale in a family study of obsessive-compulsive disorder. Depression and Anxiety, 1-19.

Damecour, C. L., \& Charron, M (1998). Hoarding: a symptom, not a syndrome. Journal of Clinical Psychiatry, 59, 267-272.

Dar, K.A., Iqbal, M., \& Mushtaq, A. (2017). Intolerance of uncertainty, depression, and anxiety: examining the indirect and moderating effects of worry. Asian Journal of Psychiatry, 29, 129-133. 
Daruna, J. H., \& Barnes, P. A. (1993). The impulsive client: Theory, research, and treatment. In W. G. McCown, J. L. Jounson, \& M. B. Shure (Eds.). A neurodevelopmental view of impulsivity. Washington, DC: American Psychological Association.

Deckersbach, T., Otto, M.W., Savage, C.R., Baer, L., \& Jenike, M.A. (2000). The relationship between semantic organization and memory in obsessive-compulsive disorder. Psychotherapy and Psychosomatics, 69, 101-107.

de Geus, F., Denys, D.A., Sitskoorn, M.M., \& Westenberg, H.G. (2007). Attention and cognition in patients with obsessive-compulsive disorder. Psychiatry and Clinical Neurosciences, 61(1), 45-53.

de Kort, M. (2012). Autistic traits in hoarding disorder: A comparative study between patients with hoarding disorder and obsessive-compulsive disorder. Unpublished master's thesis, Utrecht University, Utrecht, Netherlands.

Del Casale, A., Kotzalidis, G.D., Rapinesi, C., Serata, D., Ambrosi, E., Simonetti, A., et al. (2011). Functional neuroimaging in obsessive-compulsive disorder. Neuropsychobiology, 64(2), 61-85.

Delis, D. C., Kramer, J. H., Kaplan, E., \& Ober, B. A. (1987). California Verbal Learning Test: Adult Version Manual. San Antonio, TX: Pearson Assessment.

Dhuri, C.V., \& Parkar, S.R. (2014). Role of life events in the onset of obsessive compulsive disorder. Sri Lanka Journal of Psychiatry, 5(1), 10-13.

Didie, E.R., Menard, W., Stern, A.P., \& Phillips, K.A. (2008). Occupational functioning and impairment in adults with body dysmorphic disorder. Comprehensive Psychiatry, 24, 2628.

Diedrich, A., Sckopke, P., Schwartz, C., Schlegl, S., Osen, B., Stierle, C., et al. 
(2016). Change in obsessive beliefs as predictor and mediator of symptom change during treatment of obsessive-compulsive disorder - a process-outcome study. BMC Psychiatry, $16,220$.

Diefenbach, G. J., DiMauro, J., Frost, R., Steketee, G., \& Tolin, D.F. (2013). Characteristics of hoarding in older adults. American Journal of Geriatric Psychiatry, 21, 1043-1047.

DiMauro, J., Genova, M., Tolin, D. F., \& Kurtz, M. M. (2014). Cognitive remediation for neuropsychological impairment in hoarding disorder: a pilot study. Journal of ObsessiveCompulsive and Related Disorders, 3, 132-138.

Dittrich, W. H., \& Johansen, T. (2013). Cognitive deficits of executive functions and decisionmaking in obsessive-compulsive disorder. Scandinavian Journal of Psychology, 54(5), 393-400.

Dittrich, W.H., Johansen, T., Landro, N.I., \& Fineberg, N.A. (2011). Cognitive performance and specific deficits in OCD symptom dimensions: III, decision-making and impairments in risky choices. German Journal of Psychiatry, 14, 13-25.

Dittrich, W.H., Johansen, T., Padhi, A.K., Smith, I.E., Chamberlain, S.R., \& Fineberg, N.A. (2010).Clinical and neurocognitive changes with modafinil in obsessive-compulsive disorder: a case report. Psychopharmacology, 212, 449-451.

Dold, M., Aigner, M., Lanzenberger, K., \& Kasper, S. (2013). Antipsychotic augmentation of serotonin reuptake inhibitors in treatment-resistant obsessive-compulsive disorder: a meta-analysis of double-blind, randomized, placebo-controlled trials. International Journal of Neuropsychopharmacology, 16(3), 557-574.

Dong, Y., \& Peng, CY.J. (2013). Principled missing data methods for researchers. Springer Plus, 2(1), 132-222. 
Dozier, M.E., Porter, B., \& Ayers, C.R. (2015). Age of onset and progression of hoarding symptoms in older adults with hoarding disorder. Aging and Mental Health, 20 (7), 736742.

Dugas, M.J., Freeston, M.H., \& Ladouceur, R. (1997). Intolerance of uncertainty and problem orientation in worry. Cognitive Therapy and Research, 21, 593-606.

Dunai, J., Labuschagne, I., Castle, D. J., Kyrios, M., \& Rossell, S. L. (2010). Executive function in body dysmorphic disorder. Psychological Medicine, 40(9), 1541-1548.

Eaton, W.W., Martins, S.S., Nestadt, G., Bienvenu, O.J., Clarke, D., \& Alexandre. P. (2008) The burden of mental disorders. Epidemiologic Reviews, 30, 1-14.

Eddy, K.T., Dutra, L., Bradley, R., \& Western, D. (2004). A multidimensional metaanalysis of psychotherapy and pharmacotherapy for obsessive-compulsive disorder. Clinical Psychology Review, 24, 1011-1030.

Eisen, J.L., Phillips, K.A., Cole, M.E., \& Rasmussen, S.A. (2004). Insight in obsessive compulsive disorder and body dysmorphic disorder. Comprehensive Psychiatry, 45(1), 10-15.

Eisenberg, D.T., Mackillop, J., Modi, M., Beauchemin, J., Dang, D., Lisman, S.A., et al. (2007). Examining impulsivity as an endophenotype using a behavioral approach: a DRD2 Taql A and DRD4 48-bp VNTR association study. Behavioral and Brain Functions, 3(2), 2-4.

Ecker, W \& Gönner, S. (2008). Incompleteness and harm avoidance in OCD symptom dimensions. Behaviour research and therapy, 46, 895-904.

Eley, T.C., Lichtenstein, P., \& Moffitt, T.E. (2003). A longitudinal behavioral genetic analysis of the etiology of aggressive and nonaggressive antisocial behavior. Development and Psychopathology, 15(2), 383-402. 
Enders, C.K. (2003). Using the expectation maximization algorithm to estimate coefficient alpha for scales with item-level missing data. Psychological Methods, 8(3), 322-337.

Endicott, J., Nee, J., Harrison, W., \& Blumenthal, R. (1993). Quality of life enjoyment and satisfaction questionnaire: a new measure. Psychopharmacology Bulletin, 29, 321-326.

Evenden, J.L. (1999). Varieties of impulsivity. Psychopharmacology, 146(4), 348-361.

Exner, C., Kohl, A., Zaudig, M., Langas, G., Lincoln, T. M., \& Rief, W. (2009). Metacognition and episodic memory in obsessive-compulsive disorder. Journal of Anxiety Disorders, $23,624-631$.

Filed, A.P. (2013). Discovering statistics using IBM SPSS Statistics. London: Sage.

Fineberg, N. A., Chamberlain, S. R., Goodriaan, A. E., Stein, D. J., Louk, J. M., Potenza, M. N., et al. (2014). New developments in human neurocognition: clinical, genetic and brain imaging correlates of impulsivity and compulsivity. CNS Spectrum, 19(1), 69-89.

Fineberg, N. A., Potenza, M. N., Chamberlain, S. R., Berlin, H. A., Menzies, L., Hollander, E., et al. (2010). Probing compulsive and impulsive behaviors from animal models to endophenotypes: a narrative review. Neuropsychopharmacology, 35(3), 591-604.

First, M.B., Spitzer, R.L., \& Gibbon, M. (1996). Structured Clinical Interview for DSM-IV Axis I Disorders, Clinical Version (SCID-CV). Washington, DC: American Psychiatric Press.

Fitch, K.E. (2011). Information processing deficits in nonclinical compulsive hoarding. Unpublished master's thesis, Florida State University, Tallahassee, Florida, USA.

Flessner, C. A., Conelea, C. A., Woods, D. W., Franklin, M. E., Keuthen, N. J., \& Cashin, S. E. (2008). Styles of pulling in trichotillomania: exploring differences in symptom severity, phenomenology, and functional impact. Behavior Research and Therapy, 46(3), 345-357.

Flessner, C. A., Knopik, V. S., \& McGeary, J. (2012). Hair pulling disorder (trichotillomania): 
genes, neurobiology, and a model for understanding impulsivity and compulsivity. Psychiatry Research, 199, 151-158.

Flessner, C. A., \& Woods, D. W. (2006). Phenomenological characteristics, social problems, and the economic impact associated with chronic skin picking. Behavior Modifications, 30, 944-963.

Flessner, C. A., Woods, D. W., Franklin, M. E., Keuthen, N. J., \& Piacentini, J. (2009). Crosssectional study of women with trichotillomania: a preliminary examination of pulling styles, severity, phenomenology, and functional impact. Child Psychiatry and Human Development, 40, 153-167.

Foa, E.B., Huppert, J.D., Leiberg, S., Langner, R., Kichic, R., Hajcak, G., et al. (2002). The obsessive-compulsive inventory: development and validation of a short version. Psychological Assessment, 14(4), 485-496.

Freedman, M., (1990). Object alternation and orbitofrontal system in Alzheimer's and Parkinson's disease. Brain and Cognition, 14, 134 - 143.

Freeston, M., Rheaume, J., Letarte, H., Dugas, M.J., \& Ladouceur, R. (1994). Why do people worry? Personality and Individual Differences, 17, 791-802.

Freud, S. (1908). Character and anal erotism. The Standard Edition of the Complete Psychological Works of Sigmund Freud, (Volume 9). London, Hogarth Press.

Freyer, T., Kloppel, S., Tuscher, O., Kordon, A., Zurowski, B., Kuelz, A. K., et al. (2011). Frontostriatal activation in patients with obsessive-compulsive disorder before and after cognitive behavioral therapy. Psychological Medicine, 41, 207-216.

Frost, R. O., \& Hartl, T. L. (1996). A cognitive-behavioural model of compulsive hoarding. Behavior Research and Therapy, 34(4), 341-350. 
Frost, R.O., Lahart, C.M., \& Rosenblate, R. (1991). The development of perfectionism: A study of daughters and their parents. Cognitive Therapy and Research, 15, 469-489.

Frost, R. O., Marten, P., Lahart, C., \& Rosenblate, R. (1990). The dimensions of perfectionism. Cognitive Therapy and Research, 14(5), 449-468.

Frost, R. O., \& Shows, D. L. (1993). The nature and measurement of compulsive indecisiveness. Behavior Research and Therapy, 31(7), 683-692.

Frost, R.O., \& Steketee, G. (1997). Perfectionism in obsessive-compulsive disorder patients. Behavior Research and Therapy, 35(4), 291-296.

Frost, R.O., \& Steketee, G. (2002). Cognitive approaches to obsessions and compulsions: Theory, assessment and treatment. Oxford: Elsevier.

Frost, R. O., Steketee, G., \& Grisham, J. (2004). Measurement of compulsive hoarding: Saving inventory- revised. Behavior Research \& Therapy, 42, 1163-1182.

Frost, R. O., Steketee, G., \& Tolin, D. F. (2011). Comorbidity in hoarding disorder. Depression and Anxiety, 28, 876-884.

Frost, R. O., Steketee, G., Tolin, D. F., \& Renaud, S. (2008). Development and validation of the clutter image rating. Journal of Psychopathology and Behavior Assessment, 30, 193-203.

Fujii, D. E., Wylie, A. M., \& Nathan, J. H. (2004). Neurocognition and long-term prediction of quality of life in outpatients with severe and persistent mental illness. Schizophrenia Research, 69, 67-73.

Glass, G.V., Peckham, P.D., \& Sanders, J.R. (1972). Consequences of failure to meet assumptions underlying the fixed effects analyses of variance and covariance. Review of Educational Research, 42, 237.

Golden, C. J. (1976). Identification of brain disorders by the Stroop color and word test. Journal 
of Clinical Psychology, 32, 654-658.

Golomb, R., Franklin, M., Grant, J.E., Keuthen, N.J., Mansueto, C.S., Mouton-Odum, S., et al. (2016). Expert consensus treatment guidelines body-focused repetitive behaviors hair pulling, skin picking, and related disorders. Santa Cruz, CA: The TLC Foundation for Body-Focused Repetitive Behaviors.

Goodman, W. K., McDougle, C. J., \& Price, L.H. (1990). Beyond the serotonin hypothesis: a role for dopamine in some forms of obsessive-compulsive disorder? Journal of Clinical Psychiatry, 51, 36-43.

Goodman, W. K., Price, L. H., Rasmussen, S. A., Mazure, C., Fleischmann, R. L., Hill, C. L., et al. (1989). The Yale-Brown Obsessive Compulsive Scale. I. Development, use and reliability. Archives of General Psychiatry, 46, 1006-1011.

Gottesman, I. I., \& Gould, T. D. (2003). The endophenotype concept in psychiatry: etymology and strategic intentions. American Journal of Psychiatry, 160, 636-645.

Gottesman, I. I., \& Shields, J. (1972). Schizophenia and genetics: A twin study vantage point. New York: Academic.

Gottlich, M., Kramer, U.M., Kordon, A., Hohagen, F., \& Zurowski, B., (2015). Resting-state connectivity of the amygdala predicts response to cognitive behavioral therapy in obsessive compulsive disorder. Biological Psychology, 111, 100-109.

Grados, M. A., Atkins, E. B., Kovacikova, G., \& McVicar, E. (2015). A selective review of glutamate pharmacological therapy in obsessive-compulsive and related disorders. Psychology Research \& Behavior Management, 8, 115-131.

Grant, J. E., \& Christenson, G. A. (2007). Examination of gender in pathologic grooming behaviors. Psychiatric Quarterly, 78(4), 259-267. 
Grant, J. E., Levine, L., Kim, D., \& Potenza, M. N. (2005). Impulse control disorders in adult psychiatric inpatients. American Journal of Psychiatry, 162, 2184-2188.

Grant, J. E., Odlaug, B. L., \& Chamberlain, S. R. (2011). A cognitive comparison of pathological skin picking and trichotillomania. Journal of Psychiatric Research, 45, 1634-1638.

Grant, J. E., Odlaug, B. L., Chamberlain, S. R., \& Kim, S. W. (2010). A double-blind, placebocontrolled trial of lamotrigine for pathological skin picking: treatment efficacy and neurocognitive predictors of response. Journal of Clinical Psychopharmacology, 30(4), 396-403.

Grant, J.E., Odlaug, B.L., Hampshire, A., Schreiber, L.R., \& Chamberlain, S.R. (2013). White matter abnormalities in skin picking disorder: a diffusion tensor imaging study. Neuropsychopharmacology: official publication of the American College of Neuropsychopharmacology, 38(5), 763-769.

Grant, J. E., Odlaug, B. L., \& Kim, S. W. (2007). Lamotrigine treatment of pathological skin picking: an open -label study. Journal of Clinical Psychiatry, 68, 1384-1391.

Grant, J. E., Odlaug, B. L., \& Kim, S. W. (2009). N-Acetylcysteine, a glutamate modulator, in the treatment of trichotillomania: a double-blind, placebo-controlled study. Archives of General Psychiatry, 66, 756-763.

Green, M. F., Kern, R. S., \& Braff, D. L. (2000). Neurocognitive deficits and functional outcomes in schizophrenia: are we measuring the right stuff? Schizophrenia Bulletin, 26, 119-136.

Grisham, J. R., Brown, T. A., Savage, C. R., Steketee, G., \& Barlow, D. (2007). Neuropsychological impairment associated with compulsive hoarding. Behavior Research and Therapy, 45(7), 1471-1483.

Grisham, J., Frost, R.O., Steketee, G., \& Hood, S. (2006). Age of onset in compulsive 
hoarding. Journal of Anxiety Disorders, 20, 675-686.

Grisham, J.R., Fullana, M.A., Mataix-Cols, D., Moffitt, T.E., Caspi, A., \& Poulton, R. (2011) Risk factors prospectively associated with adult obsessive-compulsive symptom dimensions and obsessive-compulsive disorder. Psychological Medicine, 41(12), 25062595.

Grisham, J. R., Norberg, M. M., Williams, A. D., Certoma, S. P., \& Kadib, R. (2010). Categorization and cognitive deficits in compulsive hoarding. Behavior Research and Therapy, 48, 866-872.

Gross-Isseroff, R., Sasson, Y., Voet, H., Hendler, T., Luca-Haimovici, K., Kandel-Sussman H., et al. (1996). Alternation learning in obsessive-compulsive disorder. Biological Psychiatry, 39, 733-738.

Guidano, V.F., \& Liotti, G. (1983). Cognitive processes and emotional disorders. New York: Guilford.

Gunstad, J., \& Phillips, K. A. (2003). Axis 1 comorbidity in body dysmorphic disorder. Comprehensive Psychiatry, 44(4), 270-276.

Gupta, M. A., Gupta, A. K., \& Haberman, H. F., (1986). Neurotic excoriations: a review and some new perspectives. Comprehensive Psychiatry, 27, 381-386.

Gupta, M.A., Gupta, A.K., \& Knapp, K. (2015). Trichotillomania: demographic and clinical features from a nationally representative US sample. Skinmed, 13(6), 455-460.

Hanes, K.R. (1998). Neuropsychologial performance in body dysmorphic disorder. Journal of International Neuropsychological Society, 4, 167-171.

Hanna, G.L., Fischer, D.J., Chadha, K.R., Himle, J.A., \& Van Etten, M. (2005). Familial and sporadic subtypes of early-onset obsessive-compulsive disorder. Biological Psychiatry, 
$57,895-900$.

Harries, M.D., Chamberlain, S.R., Redden, S.A., Odlaug, B.L., Blum, A.W., \& Grant, J.E. (2017). A structural MRI study of excoriation (skin-picking) disorder and its relationship to clinical severity. Psychiatry Research: Neuroimaging, 269, 26-30.

Harris, D.R., Bisbee, C.T., \& Evans, S.H. (1971), Misuse of analysis of covariance. Psychological Bulletin, 75, 220-222.

Hartl, T.L., Duffany, S.R., Allen, G.J., Steketee, G., \& Frost, R.O. (2005). Relationships among compulsive hoarding, trauma, and attention-deficit/hyperactivity disorder. Behavior Research and Therapy, 43(2), 269-276.

Hartl, T. L., Frost, R. O., Allen, G. J., Deckersbach, T., Steketee, G., Duffany, S. R., et al. (2004). Actual and perceived memory deficits in individuals with compulsive hoarding. Depression and Anxiety, 20(2), 59-69.

Hayes, S. I., Storch, E. A., \& Berlanga, I. (2009). Skin picking behaviors: an examination of the prevalence and severity in a community sample. Journal of Anxiety Disorders, 23, 314319.

Heaton, R. K., Chelune, G. J., Talley, J. L., Kay, G. G., \& Curtiss, G. (1993). Wisconsin Card Sorting Test manual: Revised and expanded. Odessa, FL: Psychological Assessment Resources.

Henrich, J., Heine, S.J., \& Norenzayan, A. (2010). The weirdest people in the world? Behavioral and Brain Sciences, 33, 61-83.

Henry, J. D. (2006). A meta-analytic review of the Wisconsin Card Sorting Test and verbal fluency performance in obsessive-compulsive disorder. Cognitive Neuropsychiatry, 11(2), 156-176. 
Hermans, D., Engelen, U., Grouwels, L., Joos, E., Lemmens, J., \& Pieters, G. (2008). Cognitive confidence in obsessive-compulsive disorder: distrusting perception, attention and memory. Behavior Research and Therapy, 46(1), 98-113.

Hewitt, P.L., \& Flett, G.L. (1991). Perfectionism in the self and social contexts: conceptualization, assessment, and association with psychopathology. Journal of Personality and Social Psychology, 60, 456-470.

Hilsenroth, M.J., \& Segal, D.L. (Eds.). (2004). Comprehensive handbook of psychological assessment, Volume 2. Personality assessment. Hoboken, NJ: John Wiley \& Sons Inc.

Holdnack, H.A. (2001). Wechsler Test of Adult Reading: WTAR. San Antonio, TX: The Psychological Corporation.

Hollander, E. (2005). Obsessive-compulsive disorder and spectrum across the life span. International Journal of Psychiatry in Clinical Practice, 9, 79-86.

Holm, S. (1979). A simple sequentially rejective multiple test procedure. Scandinavian Journal of Statistics, 6(2), 65-70.

Hudziak, J.J., Van Beijsterveldt, C., Althoff, R.R., Stanger, C., Rettew, D.C., Nelson, E.C., et al. (2004). Genetic and environmental contributions to the child behavior checklist obsessive-compulsive scale: a cross-cultural twin study. Archives of General Psychiatry, 61, 608-616.

Hur, Y.M., \& Jeong, H.U. (2008). Sex differences in genetic and environmental influences on obsessive-compulsive symptoms in South Korean adolescent and young adult twins. Twin Research and Human Genetics, 11, 314-320.

Hymas, N., Lees, A., Bolton, D., Epps, K., \& Head, D. (1991). The neurology of obsessional slowness. Brain, 114, 2203-2233. 
Inouye, E. (1965). Similar and dissimilar manifestations of obsessive-compulsive neurosis in monozygotic twins. American Journal of Psychiatry, 121, 1171-1175.

Isper, J.C., Sander, C., \& Stein, D.J. (2009). Pharmacotherapy and psychotherapy for body dysmorphic disorder. Cochrane Database System Review, 21(1), 37-49.

Jamison, K.R. (2000). Suicide and bipolar disorder. Journal of Clinical Psychiatry, 61 Suppl 9, 47-51.

Jefferies-Sewell, K., Chamberlain, S.R., Fineberg, N.A., \& Laws, K.R. (2017). Cognitive dysfunction in body dysmorphic disorder: new implications for nosological systems and neurobiological models. CNS Spectrums, 22(1), 51-60.

Johannes, S., Wieringa, B. M., Mantey, M., Nager, W., Rada, D., Muller-Vahl K. R., et al. (2001). Altered inhibition of motor responses in Tourette syndrome and obsessivecompulsive disorder. Acta Neurologica Scandinavica, 104, 36-43.

Jones, E. (1918). Anal erotic character traits. The Journal of Abnormal Psychology, 13(5), 261284.

Jonnal, A.H., Gardner, C.O., Prescott, C.A., \& Kendler, K.S. (2000). Obsessive and compulsive symptoms in a general population sample of female twins. American Journal of Medical Genetics-Neuropsychiatric Genetics, 96(6), 791-796.

Julien, D., Careau, Y., O’Conner, K. P., Bouvard, M., Rheaume, J., Langlois, F., et al. (2008). Specificity of belief domains in OCD: validation of the French version of the Obsessive Beliefs Questionnaire and a comparison across samples. Journal of Anxiety Disorders, 22, 1029-1041.

Kalanthroff, E., Teichert, T., Wheaton, M.G., Kimeldorf, M.B., Linkovski, O., Ahmari, S., et al. 
(2016).The role of response inhibition in medicated and unmedicated obsessivecompulsive disorder patients: evidence from the stop-signal Task, Depression and Anxiety, 34(3), 301-306.

Katerberg, H., Delucchi, K.L., Stewart, S.E., Lochner, C., Denys, D.A., Stack, D.E., et al. (2010). Symptom dimensions in OCD: item-level factor analysis and heritability estimates. Behavior Genetics, 40(4), 505-517.

Katzman, M.A., Bleau, P., Blier, P., Chokka, P., Kjernisted, K., \& Van Ameringen, M. (2014). Canadian anxiety guidelines initiative group on behalf of the Anxiety Disorders Association of Canada: Canadian clinical practice guidelines for the management of anxiety, posttraumatic stress and obsessive-compulsive disorders. BMC Psychiatry, 14 Suppl 1, S1.

Kellner, M. (2010). Drug treatment of obsessive-compulsive disorder. Dialogues in Clinical Neuroscience, 12(2), 187-197.

Kendler, K. S., \& Neale, M. C. (2010). Endophenotype: a conceptual analysis. Molecular Psychiatry, 15, 789-797.

Kendler, K.S., \& Prescott, C.A. (2000). A population-based twin study of lifetime major depression in men and women. Archives of General Psychiatry, 57, 94-95.

Kessler, R. C., Adler, L., Ames, M., Demler, O., Faraone, S., Hiripi, E., et al. (2005). The World Health Organization adult ADHD self-report scale (ASRS): a short screening scale for the use in the general populations. Psychological Medicine, 35(2), 245-256.

Keuthen, N. J., Koran, L. M., Aboujaoude, E., Large, M. D., \& Serpe, R. T. (2010). The prevalence of pathologic skin picking in US adults. Comparative Psychiatry, 51, 183186. 
Keuthen, N. J., Makris, N., \& Schlerf, J. E. (2007). Evidence for reduced cerebellar volumes in trichotillomania. Biological Psychiatry, 61, 374-381.

Keuthen, N.J., O’Sullivan, R.L., Ricciardi, J.N., Shera, D., Savage, C.R., Borgmann, A.S., et al. (1995). The Massachusetts General Hospital (MGH) Hairpulling Scale: 1. development and factor analyses. Psychotherapy and Psychosomatics, 64, 141-145.

Keuthen, N. J., Savage, C. R., O’ Sullivan, R. L., Brown, H. D., \& Shera, D. M. (1996). Neuropsychological functioning in trichotillomania. Biological Psychiatry, 39(8), 747749.

Keuthen, N. J., Wilhelm, S., Deckersbach, T., Engelhard, I. M., Forker, A. E., Baer, L., et al. (2001). The skin picking scale: scale construction and psychometric analyses. Journal of Psychosomatic Research, 50, 337-341.

Kim, H.J., Steketee, G., \& Frost, R.O. (2001). Hoarding by elderly people. Health and Social Work, 26, 176-184.

Kitis, A., Akdede, B. B., Alpetkin, D. B., Akvardar, Y., Arkar, H., Erol, A., \& Kaya, N. (2007). Cognitive dysfunction in patients with obsessive-compulsive disorder compared to patients with schizophrenia patients: relation to overvalued ideas. Progress in NeuroPsychopharmacology and Biological Psychiatry, 31, 254-261.

Kline, R. B. (2005). Principle and practice of structural equation modeling. New York: Guilford.

Knofczynski, G.T., Gregory, T., \& Mundfrom, D. (2008). Sample sizes when using multiple linear regression for prediction. Educational and Psychological Measurement, 68(3), 431-442.

Koçak, O.M., Nalçaci, E., Özgüven, H.D., Nalçaci, E.G., \& Ergenç, İ. (2010). Evaluation of 
cognitive slowing in OCD by means of creating incongruence between lexicon and prosody. Psychiatry Research, 179, 306-311.

Koran, L, M., Abujaoude, E., Large, M. D., \& Serpse, R. T. (2008). The prevalence of body dysmorphic disorder in the United States adult population. CMS Spectrums, 13(4), 316322.

Kreuger, R. F., \& Eaton, N. R. (2015). Transdiagnostic factors of mental disorders. World Psychiatry, 14(1), 27-29.

Kruger, R.F., \& Markon, K.E. (2006). Reinterpreting comorbidity: a model-based approach to understanding and classifying psychopathology. Annual Review of Clinical Psychology, 2, 111-133.

Krikorian, R., Zimmerman, M. E., \& Fleck, D. E. (2004). Inhibitory control in obsessivecompulsive disorder. Brain \& Cognition, 54, 257-259.

Krishna, R., Udpa, S., George, C.M., Kumar, K.J., Vinswanath, B., Kandavel, T., et al. (2011). Neuropsychological performance in OCD: a study in medication-naïve patients. Progress in Neuro-Psychopharmacology and Biological Psychiatry, 35 (8), 1969-1976.

Kuelz, A. K., Hohagen, F., \& Vonderholzer, U. (2004). Neuropsychological performance in obsessive-compulsive disorder: a critical review. Biological Psychology, 65(3), 185-236.

Kurtz, M.M., Moberg, P.J., Gur, R.C., \& Gur, R.E. (2001). Approaches to cognitive remediation of neuropsychological deficits in schizophrenia: a review and meta-analysis. Neuropsychology Review, 11(4), 197-210.

Lacerda, A.L., Dalgalarrondo, P., Caetano, D., Haas, G.L., Camargo, E.E., \& Keshavan, M.S. 
(2003).Neuropsychological performance and regional cerebral blood flow in obsessivecompulsive disorder. Progress in Neuro-Psychopharmacology and Biological Psychiatry, 27, 657-665.

Landau, D., Iervolino, A.C., \& Pertusa, A. (2011). Stressful life events and material deprivation in hoarding disorder. Journal of Anxiety Disorders, 25(2), 192-202.

Lange, L. A., Kampov-Polevoy, A. B., \& Garbutt, J. C. (2010). Sweet liking and high novelty seeking: independent phenotypes associated with alcohol-related problems. Alcohol and Alcoholism, 45(5), 431-436.

Laniti, I. (2005). Neuropsychological performance of individuals with body dysmorphic disorder and obsessive compulsive disorder. Dissertation Abstracts International, 66 Section B, 2828.

Lauriola, M., Mosca, O., Trentini, C., Foschi, R., Tambelli, R., \& Carleton, R.N. (2018). The Intolerance of Uncertainty Inventory: validity and comparison of scoring methods to assess individuals screening positive for anxiety and depression. Frontiers in Psychology, 9, 388.

Lawrence, N.S., Wooderson, S., Mataix-Cols, D., David, R., Speckens, A., \& Phillips, M.L. (2006). Decision making and set shifting impairments are associated with distinct symptom dimensions in obsessive-compulsive disorder. Neuropsychology, 20, 409-419.

Leboyer, M., Bellivier, F., Nosten-Bertrand, M., Jouvent, R., Pauls, D., \& Mallet, J. (1998). Psychiatric genetics: search for phenotypes. Trends in Neuroscience, 21, 102-105. Leckman, J.F., Pauls, D.L., Zhang, H., Rosario-Campos, M.C., Katsovich, L., Kidd, K.K., et al. (2003). Obsessive-compulsive symptom dimensions in affected sibling pairs with Gilles de Tourette syndrome. American Journal of Medical Genetics, 116B, 60-68. 
Lezak, M. D. (1995). Neuropsychological Assessment ( $3^{\text {rd }}$ ed.). New York: Oxford University Press.

Lezak, M. D., Howieson, D. B., Bigler, E. D., \& Tranel, D. (2012). Neuropsychological Assessment ( $\left.5^{\text {th }} e d.\right)$. New York: Oxford University Press.

Lipszyc, J., \& Schachar, R. (2010). Inhibitory control and psychopathology: a meta-analysis of studies using the stop signal task. Journal of International Neuropsychological Society, 16, 1064-1076.

Livesley, W.J., Jang, K.L., \& Vernon, P.A. (1998). Phenotypic and genetic structure of traits delineating personality disorder. Archives of General Psychiatry, 55(10), 941-948.

Lix, L.M., Keselman, J.C., \& Keselman, H.J. (1996). Consequences of assumption violations revisited: a quantitative review of alternatives to the one-way analysis of variance $\mathrm{F}$ test. Review of Educational Research, 66, 579-619.

Lochner, C., Fineberg, N. A., Zohar, J., van Ameringen, M., Juven-Wetzler, A., Carlo Altamura, A., et al. (2014). Comorbidity in obsessive-compulsive disorder (OCD): a report from the International College of Obsessive-Compulsive Spectrum Disorders (ICOCS). Comprehensive Psychiatry, 55(7), 1513-1519.

Lochner, C., Simeon, D., Niehaus, D.J.H., \& Stein, D.J. (2002). Trichotillomania and skinpicking: a phenomenological comparison. Depression and Anxiety, 15, 83-86.

Logan, G. D., Cowan, W. B., \& Davis, K. A., (1984). On the ability to inhibit simple and choice reaction time responses: a model and a method. Journal of Experimental Psychology: Human Perception and Performance, 10(2), 276-291.

Lombardo, L.E., Bearden, C.E., Barrett, J., Brumbaugh, M.S., Pittman, B., Frangou, S., et al. (2012). Trait impulsivity as an endophenotype for bipolar disorder. Bipolar Disorders, 
$14,565-570$.

Lucey, J. V., Burness, C. E., Costa, D. C., Gacinovic, S., Pilowsky, L. D., Ell, P. J., et al. (1997). Wisconsin Card Sorting Test (WCST) errors and cerebral blood flow in obsessivecompulsive disorder (OCD). British Journal of Medical Psychology, 70, 403-411.

MacDonald, P.A., Antony, M.M., MacLeod, C.M., \& Richter, M.A. (1997). Memory and confidence in memory judgments among individuals with obsessive-compulsive disorder and nonclinical controls. Behavior Research and Therapy, 35, 497-505.

Machlin, S.R., Harris, G.J., Pearlson, G.D., Hoehn-Saric, R., Jeffery, P., \& Camargo, E.E. (1991). Elevated medial-frontal cerebral blood flow in obsessive-compulsive patients: a SPECT study. American Journal of Psychiatry, 148, 1240-1242.

Mackin, R.S., Arean, P.A., Delucchi, K.L., \& Mathews, C.A. (2011). Cognitive functioning in individuals with severe compulsive hoarding behaviors and late life depression. International Journal of Geriatric Psychiatry, 26(3), 314-321.

Malone, K.M., Haas, G.L., Sweeney, J.A., \& Mann, J.J. (1995). Major depression and the risk of attempted suicide. Journal of Affective Disorders, 34(3), 173-185.

Mansueto, C. S. (1991). Trichotillomania in focus. OCD Newsletter, 5, 10-11.

Marazziti, D., Dell'Osso, L., \& Presta, S. (1999). Platelet [3H] paroxetine binding in patients with OCD-related disorders. Psychiatry Research, 89, 223-228.

Martin, A., Wiggs, C.L., Altemus, M., Rubenstein, C., \& Murphy, D.L. (1995). Working memory as assessed by subject-ordered tasks in patients with obsessive-compulsive disorder. Journal of Clinical and Experimental Neuropsychology, 17, 786-792.

Mataix-Cols, D., Alonso, P., Pifarre, J., Menchon, M., \& Vallejo, J. (2002). Neuropsychological 
performance in medicated vs unmedicated patients with obsessive-compulsive disorder. Psychiatry Research, 109, 225-264.

Mataix-Cols, D., Frost, R. O., Pertusa, A., Clark, L.A., Saxena, S., Leckman, J. E., et al. (2010). Hoarding disorder: a new disorder for DSM-V? Depression and Anxiety, 27, 556-572.

Mataix-Cols, D., Junque, C., Sanchex-Turet, M., Vsallejo, J., Verger, K., \& Barrios, M. (1999). Neuropsychological function in a subclinical obsessive-compulsive sample. Biological Psychiatry, 45, 898-904.

Mataix-Cols, D., Rosario-Campos, M.C., \& Leckman, J.F. (2005). A multidimensional model of obsessive-compusive disorder. American Journal of Psychiatry, 162, 228-238.

Mataix-Cols, D., Wooderson, S., Lawrence, N., Brammer, M. J., Speckens, A., \& Phillips, M. L. (2004). Distinct neural correlates of washing, checking, and hoarding symptom dimensions in obsessive-compulsive disorder. Archives of General Psychiatry, 61, 564576.

Mathews, C.A., Greenwood, T., Wessel, J., Azzam, A., Garrido, H., Chavira, D.A., et al. (2007). Evidence for heritable unidimensional symptom factor underlying obsessionality. American Journal of Medical Genetics, 147 (B), 676-685.

Matsunaga, H., Hayashida, K., \& Kiriike, N. (2010). Clinical features and treatment characteristics of compulsive hoarding in Japanese patients with obsessive-compulsive disorder. CNS Spectrums, 15(4), 258-265.

Mattheisen, M., Samuels, J.F., Wang, Y., Greenberg, B., Fryer, A., McCracken, J.,...Nestadt, G. (2015). Genome-wide association study in obsessive-compulsive disorder: results from the OCGAS. Molecular Psychiatry, 20(3), 337-344. 
McConaughy, S. H., \& Achenback, T. M. (1994). Comorbidity of empirically based syndromes in matched general population and clinical samples. The Journal of Child Psychology and Psychiatry, 35(6), 1141-1157.

McDougle, C. J., Goodman, W. K., \& Leckman, J. F. (1994). Haloperidol addition in fluvoxamine-refractory obsessive-compulsive disorder: a double-blind placebo-controlled study in patients with and without tics. Archives of General Psychiatry, 51, 302-308.

McEvoy, P. M., \& Mahoney, A. E. J. (2011). Achieving certainty about the structure of intolerance of uncertainty in a treatment-seeking sample with anxiety and depression. Journal of Anxiety Disorders, 25, 112-122.

McGuire, J.F., Crawford, E.A., Park, J.M., Storch, E.A., Murphy, T.K., Larson, M.J., et al. (2014). Neuropsychological performance across symptom dimensions in pediatric obsessive compulsive disorder. Depression and Anxiety, 31(12), 988-996.

McMillan, S. G., Rees, C. S., \& Destell, C. (2013). An investigation of executive functioning, attention, and working memory in compulsive hoarding. Behaviour and Cognitive Psychotherapies, 41(5), 610-625.

Menzies, L., Achard, S., Chamberlain, S. R., Fineberg, N., Chen, C., del Campo, N., et al. (2007). Neurocognitive endophenotypes of obsessive-compulsive disorder. Brain, 130, 3223-3236.

Menzies, L., Chamberlain, S.R., Laird, A.R., Thelen, S.M., Sahakian, B.J., \& Bullmore, E.T. (2008).Integrating evidence from neuroimaging and neuropsychological studies of Biobehavior Review, 32(3), 525-549.

Miller, G.A., \& Chapman, J.P. (2001). Misunderstanding analysis of covariance. Journal of Abnormal Psychology, 110(1), 40-48. 
Moffitt, T.E., Caspi, A., \& Rutter, M. (2005). Strategy for investigating interactions between measured genes and measured environments. Archives of General Psychiatry, 62(5), 473-481.

Monzani, B., Rijsdijk, F., Harris, J., \& Mataix-Cols, D. (2014). The structure of genetic and environmental risk factors for dimensional representations of DSM-5 obsessivecompulsive spectrum disorders. JAMA Psychiatry, 71(2), 182-189.

Morein-Zamir, S., Fineberg, N. A., Robbins, T. W., \& Sahakian, B. J. (2010). Inhibition of thoughts and actions in obsessive-compulsive disorder: extending the endophenotype. Psychological Medicine, 40, 263-272.

Morein-Zamir, S., Papmeyer, M., Pertusa, A., Chamberlain, S. R., Fineberg, N. A., Sahakian, B., et al. (2014). The profile of executive function in OCD hoarders and hoarding disorder. Psychiatry Research, 215(3), 659-667.

Moritz, S., Birkner, C., Kloss, M., Jacobsen, D., Fricke, S., Bothern, A., et al. (2001). Impact of comorbid depressive symptoms on neuropsychological performance in obsessivecompulsive disorder. Journal of Abnormal Psychology, 110, 653-657.

Moritz, S., Birkner, C., Kloss, M., Jahn, H., Hand, I., Haasen C., et al. (2002).Executive functioning in obsessive-compulsive disorder, unipolar depression, and schizophrenia. Archives of Clinical Neuropsychology, 17, 477-483.

Moritz, S., \& Jaeger, A. (2018). Decreased memory confidence in obsessive-compulsive disorder for scenarios high and low on responsibility: is low still too high? European Archives of Psychiatry and Clinical Neurosciences, 268, 291-299.

Muroff, J., Steketee, G., Frost, R.O., \& Tolin, D.F. (2014). Cognitive behavior therapy for 
hoarding disorder: follow-up findings and predictors of outcome. Depression and Anxiety, 31(12), 964-971.

Murphy, D.L., Timpano, K.R., Wheaton, M.G., Greenberg, B.D., \& Miguel, E.C. (2010). Obsessive-compulsive disorder and its related disorders: a reappraisal of obsessivecompulsive spectrum concepts. Dialogues in Clinical Neuroscience, 12(2), 131-148.

Myers, S. G., Fisher, P. L., \& Wells, A. (2008). Belief domains of the Obsessive Beliefs Questionnaire-44 (OBQ-44) and their specific relationship with obsessive-compulsive symptoms. Journal of Anxiety Disorders, 22, 475-484.

Nabeyama, M., Nakagawa, A., Yoshiura, T., Nakao, T., Nakatani, E., Togao, O., et al. (2008). Functional MRI study of brain activation alterations in patients with obsessivecompulsive disorder after symptom improvement. Psychiatry Research. 163, 236-247.

Nakao, T., Nakagawa, A., Yoshiura, T., Nakatani, E., Nabeyama, M., Sanematsu, H., et al. (2009). Duration effect of obsessive-compulsive disorder on cognitive function: a functional MRI study. Depression and. Anxiety, 26, 814-823.

Nedeljkovic, M., Kyrios, M., Moulding, R., \& Doron, G. (2011). Neuropsychological changes following cognitive-behavioural treatment of obsessive-compulsive disorder (OCD). International Journal of Cognitive Therapy, 4(1), 8-20.

Nedeljkovic, M., Kyrios, M., Moulding, R., Doron, G., Wainwright, K., Pantelis, C., et al. (2009). Differences in neuropsychological performance between subtypes of obsessivecompulsive disorder. Australian and New Zealand Journal of Psychiatry, 43(3), 216-226.

Nee, D. E., Wager, T. D., \& Jonides, J. (2007). Interference resolution: insights from a metaanalysis of neuroimaging tasks. Cognitive Affective Behavior Neuroscience, 7, 1-17.

Nestadt, G., Samuels, J., Riddle, M., Bienvenu, O. J., Liang, K. Y., LaBuda, M., et al. (2000). A 
family study of obsessive-compulsive disorder. Archives of General Psychiatry, 57, 358363.

Neziroglu, F., Rabinowitz, D., \& Breytman, A. (2008). Skin picking phenomenology and severity comparison. Primary Care Companion Journal of Clinical Psychiatry, 10, 306312.

Nigg, J. T. (2000). On inhibition/disinhibition in developmental psychopathology: views from cognitive and personality psychology and a working inhibition taxonomy. Psychology Bulletin, 126, 220-222.

Nielen, M. M. \& den Boer, J. A. (2003). Neuropsychological performance of OCD patients before and after treatment with fluoxetine: evidence for persistent cognitive deficits. Psychological Medicine, 33, 917-928.

Nielen, M. M., Veltman, D. J., De Jong, R., Mulder, G., \& den Boer, J. A. (2002). Decision making performance in obsessive- compulsive disorder. Journal of Affective Disorders, 69, 257-260.

Nielsen, H., Fruensgaard, K., \& Hjortshø, A. (1980). Controlled neuropsychological investigation of patients with neurotic excoriations. Psychotherapy Psychosomatics Journal, 34, 52.

Obsessive-Compulsive Cognitions Working Group (1997). Cognitive assessment of obsessivecompulsive disorder. Behavior Research and Therapy, 35, 667-681.

Obsessive-Compulsive Working Group. (2003). Psychometric validation of the obsessive belief questionnaire and the interpretation of intrusions inventory: Part I. Behavior Research and Therapy, 41(8), 863-878.

Obsessive-Compulsive Working Group (2005). Psychometric validation of the obsessive belief 
questionnaire and the interpretation of intrusions inventory: Part 2. Factor analyses and testing of a brief version. Behavior Research and Therapy, 43(11), 1527-1542.

O’Connor, K.P., Borgeat, F., Stip, E., Brault, M., Loiselle, J., \& Robillard, S. (2002). Evaluation of a cognitive-behavioral program for the management of chronic tic and habit disorders. Behavior Research and Therapy, 39, 667-681.

Odlaug, B. L., Chamberlain, S. R., \& Grant, J. E. (2010). Motor inhibition and cognitive flexibility in pathological skin picking. Progress in Neuropsychopharmacology and Biological Psychiatry, 34(1), 208-211.

Odlaug, B. L., Chamberlain, S. R., Schreiber, L. R. N., \& Grant, J. E. (2013). Where on the obsessive impulsive-compulsive spectrum does hair-pulling disorder belong? International Journal of Psychiatry in Clinical Practice, 17(4), 279-285.

Odlaug, B. I. \& Grant, J. E. (2008a). Trichotillomania and pathologic skin picking: clinical comparison with an examination of comorbidity. Annals of Clinical Psychiatry, 20, 5763.

Odlaug, B. I. \& Grant, J. E. (2008b). Clinical characteristics and medical complications of pathologic skin-pickers. Psychosomatics, 41, 210-215.

Odlaug, B.L., Hampshire, A., Chamberlain, S.R., \& Grant, J.E. (2016). Abnormal brain activation in excoriation (skin-picking) disorder: evidence from an executive planning fMRI study. British Journal of Psychiatry, 34(1), 208-211.

Oglesby, M.E., Medley, A.N., Norr, A.M., Capron, D.W., Korte, K.J., \& Schmidt, N.B. (2013). Intolerance of uncertainty as a vulnerability factor for hoarding behaviors. Journal of Affective Disorders, 145(2), 227-231.

Okasha, A., Rafaat, M., Mahallawy, N., El Nahas, G., El Dawla, A. S., Sayed, E., et al. (2000). Cognitive dysfunction in obsessive-compulsive disorder. Acta Psychiatrica Scandina- 
vica, 101, 281-285.

Olesen, J., Gustavsson, A., Svensson, M., Wittchen, H. U., \& Jonsson, B. (2012). The economic cost of brain disorders in Europe. European Journal of Neurology, 19,155-162.

Olley, A., Malhi, G., \& Sachdev, P. (2007). Memory and executive functioning in OCD: A selected review. Journal of Affective Disorders, 104, 15-23.

Ong, C., Pang, A., Sagayadevan, V., Chong, S. A., \& Subramaniam, M. (2015). Functioning and quality of life in hoarding: a systematic review. Journal of Anxiety Disorders, 32, 17-30.

Osterrieth, P.A. (1944). Filetest de copie d'une figure complex: contribution a l'etude de la perception et de la memoire. Archives de Psychologie, 30, 286-356.

O’Sullivan, R. L., \& Christenson, G. A. (1999). Pharmacotherapy of trichotillomania. In D.J. Stein, G.A. Christenson, \& E. Hollander (Eds.), Trichotillomania (pp. 93-124). Washington, DC: American Psychiatric Press.

O’Sullivan, R. L., Phillips, K. A., Keuthen, N. J., \& Wilhelm, S. (1999). Near-fatal skin picking from delusional body dysmorphic disorder responsive to fluvoxamine. Psychosomatics, $40,79-81$.

O’Sullivan, R. L., Rauch, S. L., Breiter, H. C., Grachev, I. D., Baer, L., Kennedy, D. N., et al. (1997). Reduced basal ganglia volumes in trichotillomania measured via morphometric magnetic resonance imaging. Biological Psychiatry, 42(1), 39-45.

Owen, A. M., Downes, J. J., Sahakian, B. J., Polkey, C. E., \& Robbins, T. W. (1990). Planning and spatial working memory following frontal lobe lesions in man. Neuropsychologia, 28, 1021-1034.

Pallanti, S., Grassi, G., Sarrecchia, E.D., Cantisani, A., \& Pellegrini, M. (2011). Obsessivecompulsive disorder comorbidity : clinical assessment and therapeutic implications. 
Frontiers in Psychiatry, 2, 70.

Pallanti, S., Hollander, E., Bienstock, C., Koran, L., Leckman, J., Marazziti, D., et al. (2002). International treatment refractory OCD consortium. Treatment non-response in OCD: methodological issues and operational definitions. International Journal of Neuropsychopharmacology, 5(2):181-91.

Patton, J. H., Stanford, M. S., \& Barratt, E. S. (1995). Factor structure of the Barratt impulsiveness scale. Journal of Clinical Psychology, 51(6), 768-774.

Paul, M.A., Gray, G.W., Love, R.J., \& Lange M. (2007). SSRI effects on psychomotor performance: assessment of citalopram and escitalopram on normal subjects. Aviation Space and Environmental Medicine, 78(7), 693-697.

Pauls, D.L. (2010). The genetics of obsessive-compulsive disorder: a review. Dialogues in Clinical Neuroscience, 12(2), 149-163.

Pauls, D. L., Alsobrook, J. P., Goodman, W., Rasmussen, S., \& Leckman, J. F. (1995). A family study of obsessive compulsive disorder. American Journal of Psychiatry, 152, 76-84.

Pedersen, N.L., Plomin, R., McClearn, G.E., \& Friberg, L. (1988). Neuroticism, extraversion, and related traits in adult twins reared apart and reared together. Journal of Personality and Social Psychology, 55(6), 950-957.

Penades, R., Catalan, R., Rubia, K., Andres, S., Salamero, M., \& Gasto, C. (2007). Impaired response inhibition in obsessive compulsive disorder. European Psychiatry 22, 404-410.

Perez-Vigil, A., De La Cruz, L.F., Brander, G., Isomura, K., Jangmo, A., Feldman, I.,...MataixCols, D. (2018). Association of obsessive-compulsive disorder with objective indicators of educational attainment: A nationwide register-based sibling control study. JAMA Psychiatry, 75(1), 47-55. 
Pertusa, A., Fullana, M.A., \& Singh, S. (2008). Compulsive hoarding: OCD symptom, distinct clinical syndrome, or both? American Journal of Psychiatry, 165(10), 1289-1298.

Perugi, G., Akiskal, H., Giannotti, D., Frare, F., Di Valo, S., \& Cassano, G. (1997). Gender-related differences in body dysmorphic disorder (dysmorphophobia). Journal of Nervous and Mental Disease, 185(9), 578-582.

Phillips, K.A. (2000). Understanding body dysmorphic disorder: an essential guide. New York: Oxford University Press.

Phillips, K. A., Albertini, R.S., \& Rasmussen, S. A. (2002). A randomized placebo-controlled trial of fluoxetine in body dysmorphic disorder. Archives of General Psychiatry, 59(4), 381388.

Phillips, K. A., \& Diaz, S. F. (1997). Gender differences in body dysmorphic disorder. Journal of Nervous and Mental Disease, 185(9), 570-577.

Phillips, K. A., McElroy, S. L., Keck, P. E., Pope, H. G., \& Hudson, J. I. (1993). Body dysmorphic disorder: 30 cases of imagined ugliness. American Journal of Psychiatry, 150, 302-308.

Phillips, K. A., \& Menard, W. (2006). Suicidality in body dysmorphic disorder: a prospective study. American Journal of Psychiatry, 163(7), 1280-1282.

Phillips, K.A., Menard, W., Fay, C., \& Weisberg, R. (2005). Demographic characteristics, phenomenology, comorbidity, and family history in 200 individuals with body dysmorphic disorder. Psychosomatics, 46(4), 317-325.

Phillips, K. A., \& Najar, F. (2003). An open-label study of citalopram in body dysmorphic disorder. Journal of Clinical Psychiatry, 64(6), 715-720.

Pinto, A., Dargani, N., Wheaton, M.G., Cervoni, C., Rees, C.S., \& Egan, S.J. (2017). Perfectionism in obsessive-compulsive disorder and related disorders: what should treating clinicians 
know? Journal of Obsessive-Compulsive and Related Disorders, 12, 102-108.

Pittenger, C. (2015). Glutamatergic agents for OCD and related disorders. Current Treatment Options in Psychiatry, 2(3), 271-283.

Pittenger, C., Bloch, M., \& Williams, K. (2012). Glutamate abnormalities in obsessive compulsive disorder: neurobiology, pathophysiology, and treatment. Pharmacology \& Therapeutics, 132(3), 314-332.

Preston, G. A., \& Weinberger, D. R. (2005). Intermediate phenotype in schizophrenia: a selective review. Dialogues of Clinical Neuroscience, 7, 165-179.

Purcell, R., Maruff, P., Kyrios, M., \& Pantelis, C. (1998a). Cognitive deficits in obsessive compulsive disorder on tests of frontalstriatal function. Biological Psychiatry, 43, 348357.

Purcell, R., Maruff, P., Kyrios, M., \& Pantelis, C. (1998b). Neuropsychological deficits in obsessive-compulsive disorder: a comparison with unipolar depression, panic disorder, and normal controls. Archives of General Psychiatry, 55, 415, 423.

Rachman, S. (1997). A cognitive theory of obsessions. Behaviour Research and Therapy, 35, 793-802.

Radua, J., \& Mataix-Cols, D. (2009). Voxel-wise meta-analysis of grey matter changes in obsessive-compulsive disorder. British Journal of Psychiatry, 195, 393-402.

Rao, N. P., Reddy, Y. C. J., Kumar, K. J., Kandavel, T., \& Chandrashekar, C. R. (2008). Are neuropsychological deficits trait markers in OCD? Progress in NeuroPsychopharmacology \& Biological Psychiatry, 32, 1574-1579.

Rasmussen, J. L. (2012). Acquisition and impulsivity in compulsive hoarding. Unpublished doctoral dissertation, Boston University, Boston. 
Rasmussen, J. L., Brown, T. A., Steketee, G. S., \& Barlow, D. H. (2013). Impulsivity in hoarding. Journal of Obsessive-Compulsive and Related Disorders, 2, 183-191.

Rauch, S. L., Shin, L. M., \& Wright, C. I. (2003). Neuroimaging studies of amygdala function in anxiety disorders. Annals of the New York Academy of Science, 985, 389-410.

Ravizza, I., Barzega, G., Bellino, S., Bogetto, F., \& Maina, G. (1995). Predictors of drug treatment response in obsessive-compulsive disorder. The Journal of Clinical Psychiatry, 56, 368373.

Rector, N. A., Cassin, S. E., Richter, M. A., \& Burroughs, E. (2009). Obsessive beliefs in firstdegree relatives of patients with OCD: a test of the cognitive vulnerability model. Journal of Anxiety Disorders, 23, 145-149.

Rehm, I.C., Nedeljkovis, M., Thomas, A., \& Moulding, R. (2015). The role of cognitions and beliefs in trichotillomania: a qualitative study using interpretative phenomenological analysis. Behaviour Change, 32(4), 209-230.

Reuther, E.T., Davis, T.E., Rudy, B.M., Jenkins, W.S., Whiting, S.E., \& May, A.C. (2013). Intolerance of uncertainty as a mediator of the relationship between perfectionism and obsessive-compulsive symptom severity. Depression and Anxiety, 30(8), 773-777.

Rhéaume, J., Freeston, M. H., Dugas, M. J., Letarte, H., \& Ladouceur, R. (1995). Perfectionism, responsibility, and obsessive-compulsive symptoms. Behavior Research and Therapy, 33, 785-794.

Rhee, S.H., Hewitt, J.K., Lessem, J.M., Stallings, M.C., Corley, R.P., \& Neale, M.C. (2004). The validity of the Neale and Kendler model-fitting approach in examining the etiology of comorbidity. Behavioral Genetics, 34(3), 251-265.

Richter, M.M, \& Ramos, T.R. (2018). Obsessive-compulsive disorder, Behavioral Neurology 
and Psychiatry, 24(3), 828-844.

Ridley, R. M. (1994). The psychology of perseverative and stereotyped behavior. Progress in Neurobiology, 44, 221-231.

Rief, W., Buhlmann, U., Wilhelm, S., Borkenhagen, A., \& Brahler, E. (2006). The prevalence of body dysmorphic disorder: a population based survey. Psychological Medicine, 36(6), 877885.

Robbins, T. W., Gillan, C. M., Smith, D. G., de Wit, S., \& Ershe, K. D. (2012). Neurocognitive endophenotypes of impulsivity and compulsivity: towards dimensional psychiatry. Trends in Cognitive Science, 16, 81-91.

Rotge, J.Y., Guehl, D., Dilharreguy, B., Tignol, J., Bioulac, B., Allard, M.,...Aouizerate, B. (2009). Meta-analysis of brain volume changes in obsessive-compulsive disorder. Biological Psychiatry, 65, 75-83.

Rotge, J.Y., Langbour, N., Guehl, D., Bioulac, B., Jaafari, N., Allard, M.,...Burbaud, P. (2010). Gray matter alterations in obsessive-compulsive disorder: an anatomic likelihood estimation meta-analysis. Neuropsychopharmacology, 35, 686-691.

Ruscio, A. M., Stein, D. J., Chiu, W. T., \& Kessler, R. C. (2010). The epidemiology of obsessive-compulsive disorder in the National Comorbidity Survey Replication. Molecular Psychiatry, 15(1), 53-63.

Sachdev, P. S., \& Malhi, G. S. (2005). Obsessive-compulsive behaviour: a disorder of decisionmaking. Australian and New Zealand Journal of Psychiatry, 39, 757-763.

Salkovskis, P.M. (1996). The cognitive approach to anxiety: threat beliefs, safety-seeking behavior, and the special case of health anxiety and obsessions. In P.M. Salkovskis (Ed,), Frontiers of cognitive therapy (pp 48-74). New York: Guilford. 
Salkovskis, P., Shafran, R., Rachman, S., \& Freeston, M. (1999). Multiple pathways to inflated responsibility beliefs in obsessional problems. Behaviour Research and Therapy, 37, 10551072).

Samuels, J. F., Bienvenu, O. J., Grados, M. A., Cullen, B., Riddle, M. A., \& Liang, K. Y. (2008). Prevalence and correlates of hoarding behaviour in a community-based sample. Behavior Research and Therapy, 46, 836-844.

Samuels, J., Nestadt, G., Bienvenu, O. J., Costa, P. T., Riddle, M. A., Liang, K., et al. (2000). Personality disorders and normal personality dimensions in obsessive-compulsive disorder. British Journal of Psychiatry, 177, 457-462.

Sanz, M., Molina, V., \& Calcedo, A. (2001). The Wisconsin Card Sorting Test and the assessment of frontal function in obsessive-compulsive patients: an event-related potential study. Cognitive Neuropsychiatry, 6, 109-129.

Sassaroli, S., Lauro, L.J., Ruggiero, G.M., Mauri, M.C., Vinai, P., \& Frost, R. (2008). Perfectionism in depression, obsessive-compulsive disorder and eating disorders. Behaviour Research and Therapy, 46(6), 757-765.

Savage, C. R. (1997). Neuropsychology of subcortical dementias. Pediatric Clinics of North America, 20, 911-931.

Savage, C. R., Baer, L., Keuthen, N. J., Brown, H. D., Rauch, S. L., \& Jenike, M. A. (1999). Organizational strategies mediate nonverbal memory impairment in obsessivecompulsive disorder. Biological Psychiatry, 45, 905-916.

Savage, C.R., Deckersbach, T., Wilhelm, S., Rauch, S.L., Baer, L., \& Reid, T. (2000). Strategic processing and episodic memory impairment in obsessive-compulsive disorder. Neuropsychology, 14, 141-151. 
Saxena, S. (2008). Neurobiology and treatment of compulsive hoarding. CNS Spectrums, 13, 2936.

Saxena, S., Ayers, C. R., Maidment, K. M., Vapnik, T., Wetherell, J. L., \& Bystritsky, A. (2011). Quality of life and functional impairment in compulsive hoarding. Journal of Psychiatric Research, 45, 475-480.

Saxena, S., Bota, R. G., \& Brody, A. L. (2001). Brain-behavior relationships in obsessivecompulsive disorder. Seminars in Clinical Neuropsychiatry, 6, 82-101.

Schachar, R., Logan, G. D., Robaey, P., Chen, S., Ickowicz, A., \& Barr, C. (2007). Restraint and cancellation: multiple inhibition deficits in attention deficit hyperactivity disorder. Journal of Abnormal Child Psychology, 35, 229-238.

Scheffé, H. (1959). The Analysis of Variance. New York: Wiley.

Schieber, K., Kollei, I., de Zwann, M., Muller, A., \& Martin, A. (2013). Personality traits as vulnerability factors in body dysmorphic disorder. Psychiatry Research, 210, 242-246.

Schlosser, R. G., Wagner, G., Schachtzabel, C., Peikert, G., Koch, K., Reichenbach, J. R., et al. (2010). Fronto-cingulate effective connectivity in obsessive compulsive disorder: a study with MRI and dynamic causal modeling. Human Brain Mapping, 31, 1834-1850.

Schneibel, R., Brakemeier. E.L., Wilbertz, G., Dykierek, P., Zobel, I., \& Schramm, E. (2012). Sensitivity to detect change and the correlation of clinical factors with the Hamilton Depression Rating Scale and the Beck Depression Inventory in depressed inpatients. Psychiatry Research, 198, 62-67.

Sebastian, A., Pohl, M. F., Kloppel, S., Feige, B., Lange, T., Stahl, C., et al. (2013). Disentangling common and specific neural sub-processes of response inhibition. Neuroimage, 64, 601-615. 
Seroczynski, A.D., Bergeman, C.S., \& Coccaro, E.F. (1999). Etiology of impulsivity/ aggression relationship : genes or environment? Psychiatry Research, 86, 41-57.

Serretti, A., Calati, R., Goracci, A., Di Simplicio, M., Castrogiovanni, P., \& De Ronchi, D. (2010). Antidepressants in healthy subjects: what are the psychotropic/psychological effects? European Neuropsychopharmacology, 20(7), 433-453.

Sexton, K.A., \& Dugas, M.J. (2009). Defining distinct negative beliefs about uncertainty: validating the factor structure of the intolerance of uncertainty scale. Psychological Assessment, 21(2), 176-186.

Sheehan, D. V. (1983). The Anxiety Disease. New York: Charles Scribner and Sons.

Sher, K. J., Frost, R. O., \& Otto, R. (1983). Cognitive deficits in compulsive checkers: an exploratory study. Behavior Research and Therapy, 21, 357-363.

Shetti, C. N., Reddy, Y. C., Kandavel, T., Kashyap, K., Singisetti, S., Hiremath A.S., et al. (2005). Clinical predictors of drug nonresponse in obsessive-compulsive disorder. The Journal of Clinical Psychiatry, 66, 1517-1523.

Simeon, D., Stein, D. J., Gross, S., Islam, N., Schmeidler, J., \& Hollander, E. (1997). A doubleblind trial of fluoxetine in pathologic skin picking. Journal of Clinical Psychiatry, 58, 341-347.

Simpson, H.B., Rosen, W., Huppert, J.D., Lin, S.H., Foa, E.B., \& Liebowitz, M.R. (2006). Are there reliable neuropsychological deficits in obsessive-compulsive disorder? Journal of Psychiatric Research, 40, 247-257.

Skapinakis, P., Caldwell, D.M., Hollingworth, W., Bryden, P., Fineberg, N.A., Salkovskis, P., et al. (2016). Pharmacological and psychotherapeutic interventions for management of obsessive-compulsive disorder in adults: a systematic review and network meta-analysis. 
The Lancet Psychiatry, 3(8), 730-739.

Sklar, P. (2002). Linkage analysis in psychiatric disorders: the emerging picture. Annual Review of Genomics and Human Genetics, 25, 1-50.

Slade, P.D., Coppel, D.B., \& Townes, B.D. (2009). Neurocognitive correlates of positive and negative perfectionism. International Journal of Neuroscience, 119(10), 1741-1754.

Slama, F., Bellivier, F., Henry, C., Rousseva, A., Etain, B., Rouillon, F., et al. (2004). Bipolar patients with suicidal behavior: toward the identification of a clinical subgroup. Journal of Clinical Psychiatry, 65, 1035-1039.

Snorrason, I., Belleau, E.L., \& Woods, D.W. (2012). How related are hair pulling disorder (trichotillomania) and skin picking disorder? A review of evidence for comorbidity, similarities and shared etiology. Clinical Psychology Review, 32(7), 618-629.

Snorrason, I., Smári, J., \& Ólafsson, R. P. (2011). Motor inhibition, reflection impulsivity, and trait impulsivity in pathological skin picking. Behavior Therapy, 42, 521-532.

Sorensen, C. B., Kirkeby, L., \& Thomsen, P. H. (2004). Quality of life with OCD. A selfreported survey among members of the Danish OCD Association. Nordic Journal of Psychiatry, 58, 231-236.

Sorensen, T.H., Olsen, K.R., \& Vedsted, P. (2009). Association between general practice referral rates and patients' socioeconomic status and access to specialized health care: a population-based nationwide study. Health Policy, 92(2-3), 180-186.

Stairs, A.M., Smith, G.T., Zapolski, T.C., Combs, J.L., \& Settles, R.E. (2012). Clarifying the construct of perfectionism. Assessment, 19(2), 146-166. 
Stanford, M.S., Mathias, C.W., Dougherty, D.M., Lake, S.L., Anderson, N.E., \& Patton, J.H. (2009). Fifty years of the Barratt Impulsiveness Scale: an update and review. Personality and Individual Differences, 47, 385-395.

Stanley, M. A., Hannay, H. J., \& Breckenridge, J. K. (1997). The neuropsychology of trichotillomania. Journal of Anxiety Disorders, 11, 473-488.

Stanton, K., \& Watson, D. (2014). Positive and negative affective dysfunction in psychopathology. Social and Personality Psychology Compass, 8(9), 555-567.

Starcke, K., Tuschen-Caffier, B., Markowitsch, H. J., \& Brand, M. (2010). Dissociation of decisions in ambiguous and risky situations in obsessive-compulsive disorder. Psychiatry Research, 175, 114-120.

Stein, D.J., Fineberg, N.A., Bienvenu, O.J., Denys, D., Lochner, C., Nestadt, G., et al. (2010). Should OCD be classified as an anxiety disorder in DSM-V? Depression and Anxiety, 27(6), 495-506.

Stein, D. J., \& Hollander, E. (1992). Low-dose pimozide augmentation of serotonin reuptake blockers in treatment of trichotillomania. Journal of Clinical Psychiatry, 53, 123-126.

Stein, D.J., Hollander, E., Simeon, D., \& Cohen, L. (1994). Impulsivity scores in patients with obsessive-compulsive disorder. Journal of Nervous and Mental Disease, 182(4), 240241.

Stein, M. B., Forde, D. R., Anderson, G., \& Walker, J. R. (1997). Obsessive-compulsive disorder in the community: an epidemiologic survey with clinical reappraisal. American Journal of Psychiatry, 154(8), 1120-1126.

Steketee, G., \& Frost, R. (2003). Compulsive hoarding: current status of the research. Clinical Psychology Review, 23, 905-927. 
Steketee, G., Frost, R. O., \& Kim, H. J. (2001). Hoarding by elderly people. Health \& Social Work, 26, 176-184.

Steketee, G., Frost, R. O., \& Kyrios, M. (2003). Cognitive aspects of compulsive hoarding. Cognitive Therapy and Research, 27, 463-479.

Steketee, G., Frost, R. O., Tolin, D. F., Rasmussen, J., \& Brown, T. A. (2010). Waitlistcontrolled trial of cognitive behaviour therapy for hoarding disorder. Depression and Anxiety, 27(5), 476-484.

Steketee, G., Frost, R. O., Wincze, J., Greene, K., \& Douglass, H. (2000). Group and individual treatment of compulsive hoarding: a pilot study. Behavioral \& Cognitive Psychotherapies, 28, 259-268.

Stern, E. R., Welsh, R. C., Gonzalez, R., Fitzgerald, K. D., Abelson, J. L., \& Taylor, S. F. (2013). Subjective uncertainty and limbic hyperactivation in obsessive-compulsive disorder. Human Brain Mapping, 34, 1956-1970.

Sternberger, R. M. T., Thomas, A. M., Mansueto, C.S., \& Carter, J. G. (2000). Personal toll of trichotillomania: behavioral and interpersonal sequelae. Journal of Anxiety Disorders, 14, 97-104.

Stewart, S.E., Rosario, M.C., Baer, L., Carter, A.S., Brown, T.A., Scharf, J.M., et al. (2008). Four-factor structure of obsessive-compulsive disorder symptoms in child, adolescents, and adults. Journal of the American Academy of Child and Adolescent Psychiatry, 47(7), 763-772.

Stewart, S.E., Yu, D., Scharf, J.M., Neale, B.M., Fagerness, J.A., Mathews, C.A.,...Pauls, D. (2013). Genome-wide association study of obsessive-compulsive disorder. Molecular Psychiatry, 18(7), 788-798.

Stober, J. (1998). The Frost Multidimensional Perfectionism scale: more perfect with four 
(instead of six) dimensions. Personality and Individual Differences, 24, 481-491.

Storch, E.A., Abramowitz, J., \& Goodman, W.K. (2008). Where does obsessive-compulsive disorder belong in DSM-V? Depression and Anxiety, 25(4), 336-347.

Strauss, E., Sherman, E.M., \& Spreen, O, (2006). A compendium of neuropsychological tests: Administration, norms and commentary (3rd ed.). New York: Oxford University Press.

Stuss, D. T., \& Benson, D. F. (1986). The frontal lobes. New York: Raven Press.

Summerfeldt, L.J., Hood, K., Antony, M.M., Richter, M.A., \& Swinson, R.P., (2004). Impulsivity in obsessive-compulsive disorder: comparisons with other anxiety disorders and within tic-related subgroups. Personality and Individual Differences, 36(3), 539-553.

Summerfeldt, L.J., Richter, M.A., Antony, M.M., \& Swinson, R.P. (1999). Symptom structure in obsessive-compulsive disorder: a confirmatory factor-analytic study. Behaviour Research and Therapy 37, 297-311.

Sumner, J. M., Noack, C. G., Filoteo, J. V., Maddox, W. T., \& Saxena, S. (2016). Neurocognitive performance in unmedicated patients with hoarding disorder. Neuropsychology, 30(2), 157-168.

Swedo, S. E., Leonard, H. L., \& Rapoport, J. L. (1989). A double-blind comparison of clomipramine and desipramine in the treatment of trichotillomania (hair pulling). New England Journal of Medicine, 321, 497-501.

Swedo, S. E., Rapoport, J. L., \& Leonard, H. L. (1991): Regional cerebral glucose metabolism of women with trichotillomania. Archives of General Psychiatry, 48, 828-833.

Taillefer, S.E., Liu, J.J.W., Ornstein, T.J., \& Vickers, K. (2016). Indecisiveness as a predictor of quality of life in individuals with obsessive and compulsive traits. Journal of ObsessiveCompulsive and Related Disorders, 10, 91-98. 
Tambs, K., Czajkowsky, N., Neale, M.C., Reichborn-Kiennerud, T., Aggen, S.H., Harris, J.R., et al. (2009). Structure of genetic and environmental risk factors for dimensional representations of DSM-IV anxiety disorders. British Journal of Psychiatry, 195(4), 301307.

Taylor, S. (2011). Etiology of obsessions and compulsions: a meta-analysis and narrative review of twin studies. Clinical Psychology Review, 31, 1361-1372.

Taylor, S. (2012). Endophenotypes of obsessive-compulsive disorder: current status and future directions. Journal of Obsessive-Compulsive and Related Disorders, 1, 258-262.

Taylor, S. (2013). Molecular genetics of obsessive-compulsive disorder: a comprehensive metaanalysis of genetic association studies. Molecular Psychiatry, 18(7), 799-805.

Taylor, S., Abramowitz, J. S., McKay, D., \& Cutter, C. (2012). Cognitive approaches to understanding obsessive-compulsive and related disorders. In G. Steketee (Ed.), Oxford handbook of obsessive compulsive and spectrum disorders (pp. 233-250). New York: Oxford University Press.

Taylor, S., Coles, M. E., Abramowitz, J. S., Wu, K. D., Olatunji, B. O., Timpano, K. R., et al. (2010). How are dysfunctional beliefs related to obsessive-compulsive symptoms? Journal of Cognitive Psychotherapy: An International Quarterly, 24(3), 165-176.

Taylor, S., \& Jang, K. L. (2011). Biopsychosocial etiology of obsessions and compulsions: an integrated behavioral-genetic and cognitive-behavioral analysis. Journal of Abnormal Psychology, 120, 174-186.

Taylor, S., Jang, K.L., \& Asmundson, G.J. (2010). Etiology of obsessions and compulsions: a behavioral-genetic analysis. Journal of Abnormal Psychology, 119, 672-682.

Teng, E. J., Woods, D. W., \& Twohig, M. P. (2006). Habit reversal as a treatment for chronic 
skin picking: a pilot investigation. Behavior Modification, 30, 411-422.

Testa, R., Pantellis, C., \& Fontanelle, L.F. (2011). Hoarding behaviors in children with learning disabilities. Journal of Child Neurology, 12, 574-579.

Timpano, K. R., Rasmussen, J., Exner, C., Rief, W., Schmidt, N. B., \& Wilhelm, S. (2013). Hoarding and the multi-faceted construct of impulsivity: a cross-cultural investigation. Journal of Psychiatric Research, 47, 363-370.

Tolin, D.F., Abramowitz, J.S., Brigidi, B.D., \& Foa, E.B. (2003). Intolerance of uncertainty in obsessive-compulsive disorder. Journal of Anxiety Disorders, 17(2), 233-242.

Tolin, D.F., Brady, R.E., \& Hannan, S.E. (2008). Obsessional beliefs and symptoms of obsessive-compulsive disorder in a clinical sample. Journal of Psychopathology and Behavioral Assessment, 30, 31-42.

Tolin, D.F., Kiehl, K.A., Worhunsky, P., Book, G.A., \& Maltby, N. (2009). An exploratory study of the neural mechanisms of decision making in compulsive hoarding. Psychological Medicine, 39(2), 325-336.

Tolin, D.F., Meunier, S.A., Frost, R.O., \& Steketee, G. (2011). Compulsive hoarding among patients seeking treatment for anxiety disorders. Journal of Anxiety Disorders, 25, 43-48.

Tolin, D.F., Stevens, M.C., Villavicencio, A.L., Norberg, M.M., Calhoun, V.D., Frost, R.O., et al. (2012). Neural mechanisms of decision making in hoarding disorder. Archives of General Psychiatry, 69(8), 832-841.

Tolin, D.F., \& Villavicencio, A. (2011). An exploration of economic reasoning in hoarding disorder patients. Behavior Research and Therapy, 49(12), 914-919.

Tolin, D.F., Villavicencio, A., Umback, A., \& Kurtz, M.M. (2011). Neuropsychological functioning in hoarding disorder. Psychiatry Research, 189(3), 413-418. 
Tolin, D.F., Witt, S.T., \& Stevens, M.C. (2014). Hoarding disorder and obsessive-compulsive disorder show different patterns of neural activity during response inhibition. Psychiatry Research, 221(2), 142-148.

Tolin, D. F., Woods, C. M., \& Abramowitz, J. S. (2003). Relationship between obsessive beliefs and obsessive-compulsive symptoms. Cognitive Therapy and Research, 27, 657-669.

Torres, A. R., Prince, M. J., Bebbington, P. E., Bhugra, D., Brugha, T. S., Farrell, M., et al. (2006). Obsessive-compulsive disorder: prevalence, comorbidity, impact, and helpseeking in the British National Psychiatric Morbidity Survey of 2000. The American Journal of Psychiatry, 163, 1978-1985.

Valderas, J.M., Starfield, B., Sibbald, B., Salisbury, C., \& Roland, M. (2009). Defining comorbidity: implications for understanding health and health services. Annals of Family Medicine, 7(4), 357-363.

Van Ameringen, M., Mancini, M., \& Oakman, J. M. (1999). The potential role of haloperidol in the treatment of trichotillomania. Journal of Affective Disorders, 56, 219-226.

Van Ameringen, M., Mancini, M., \& Patterson, B. (2006). A randomized placebo controlled trial of olanzapine in trichotillomania. European Neuropsychopharmacology, 16, S452.

Van Ameringen, M., Patterson, B., \& Simpson, W. (2014). DSM-5 Obessive-compulsive and related disorders: clinical implications of new criteria. Depression and Anxiety, 31, 487493.

Van den Hout, M., \& Kindt, M. (2003).Repeated checking causes memory distrust. Behavior Research and Therapy, 41(3),301-316.

Van de Huevel, O. A., Van de Werf, Y., Verhoef, K. M. J., De Wit, S., \& Berendse, S. (2010). 
Fronto-striatal abnormalities underlying behaviors in the compulsive-impulsive spectrum. Journal of the Neurological Sciences, 289, 55-59.

Van Eersel, G., Bouwmeester, S., Polak, M.G., \& Verkoeijen, P. (2017). The misuse of ANCOVA in neuroimagine studies. Open Science Framework, 7, 1-16.

Van Grootheest, D.S., Bartels, M., Cath, D.C., Beekman, A.T., Hudziak, J.J., \& Boomsma, D.I. (2007). Genetic and environmental contributions underlying stability in childhood obsessive-compulsive behavior. Biologial Psychiatry, 61, 308-315.

Van Grootheest, D.S., Bartels, M., Van Beijsterveldt, C.E., Cath, D.C., Beekman, A.T., Hudziak, J.J., et al. (2008). Genetic and environmental contributions to self-report obsessivecompulsive symptoms in Dutch adolescents at ages 12, 14, and 16. Journal of the American Academy of Child and Adolescent Psychiatry, 47, 1182-1188.

Van Grootheest, D.S., Cath, D.C., Beekman, A.T., \& Boomsma, D.I. (2007). Genetic and environmental influences on obsessive-compulsive symptoms in adults: a populationbased twin-family study. Psychological Medicine, 37(11), 1635-1644.

Van Minnen, A., Hoogduin, K. A., Keijsers, G. P., Hellenbrand, I., \& Hendriks, G. J. (2003). Treatment of trichotillomania with behavioral therapy or fluoxetine: a randomized waiting-list controlled study. Archives of General Psychiatry, 60, 517-522.

Veale, D. (2004). Advances in a cognitive behavioural model of body dysmorphic disorder. Body Image, 1, 113-125.

Veale, D., Boocock, A., Gournay, K., \& Dryden, W. (1996). Body dysmorphic disorder: a survey of fifty cases. British Journal of Psychiatry, 169(2), 196-201.

Veale, D., Gournay, K., Dryden, W., Boocock, A., \& Shah, F. (1996). Body dysmorphic 
disorder: a cognitive behavioural model and pilot randomised controlled trail. Behavior Research and Therapy, 34(9), 717-729.

Veale, D., Miles, S., Smallcombe, N., Chezai, H., Goldacre, B., \& Hodsoll, J. (2014). Atypical antipsychotic augmentation in SSRI treatment refractory obsessive-compulsive disorder: a systematic review and meta-analysis. BMC Psychiatry, 14, 317.

Ventura, J., Liberman, R.P., Green, M.F., Shaner, A., \& Mintz, J. (1998). Training and quality assurance with the structured clinical interview for DSM-IV. Psychiatry Research, 79(2), 163-173.

Viswanath, B., Reddy, J., Kumar, K. J., Kandavel, T., \& Chandrashekar, C. R. (2009). Cognitive endophenotypes in OCD: a study of unaffected siblings of probands with familial OCD. Progress in Neuro-Psychopharmacology \& Biological Psychiatry, 33, 610-615.

Wager, T. D., Sylvester, C. Y., Lacey, S. C., Nee, D. E., Franklin, M., \& Jonides, J. (2005). Common and unique components of response inhibition revealed by fMRI. Neuroimage, 27, 323-340.

Watkins, L. H., Sahakian, B. J., Robertson, M. M., Veale, D. M., Rogers, R. D., Pickard, K. M., et al. (2005). Executive function in Tourette's syndrome and obsessive-compulsive disorder. Psychological Medicine, 35, 571-582.

Watson, D., Clark, L.A., \& Carey, G. (1988). Positive and negative affectivity and their relation to anxiety and depressive disorders. Journal of Abnormal Psychology, 97(3), 346-353.

Weber, R.C., Riccio, C.A., \& Cohen, M.J. (2013) Does Rey Complex Figure copy performance measure executive function in children? Applied Neuropsychology of the Child, 2, 6-12.

Wechsler, D. (1997). The Wechsler Adult Intelligence Scale ( $3^{\text {rd }}$ ed.). San Antonio, TX: The Psychological Corporation. 
Weidt, S., Klaghofer, R., \& Kuenburg, A. (2015). Internet-based self-help for trichotillomania: a randomized controlled study comparing decoupling and progressive muscle relaxation. Psychotherapy and Psychosomatics, 84(6), 359-367.

Weissman, M. M., Bland, R. C., Canino, G. J., Greenwald, S., Hwu, H. G., Lee, C., et al. (1994). The cross national epidemiology of obsessive compulsive disorder. The Cross National Collaborative Group. Journal of Clinical Psychiatry, 55, 5-10.

Wheaton, M.G., Abramowitz, J.S., Jacoby, R.J., Zwerling, J., \& Rodiguez, C.I. (2016). An investigation of the role of intolerance of uncertainty in hoarding symptoms. Journal of Affective Disorders, 193, 208-214.

Whiteside, S.P., \& Lynam, D.R. (2001). The five factor model and impulsivity: using a structural model of personality to understand impulsivity. Personality and Individual Differences, 30(4), 669-689.

Wilhelm, S. (2006). Feeling good about the way you look: A program for overcoming body image problems. New York: Guilford Press.

Wilhelm, S., Berman, N. C., Keshaviah, A., Schwartz, R. A., \& Steketee, G. (2015). Mechanism of change in cognitive therapy for obsessive compulsive disorder. Role of maladaptive beliefs and schema. Behavior Research and Therapy, 65, 5-10.

Wilhelm, S., Otto, M. W., Lohr, B., \& Deckersbach, T. (1999). Cognitive behavior group therapy for body dysmorphic disorder. Behavior Research and Therapy, 37, 71-75.

Wilhelm, S., Philips, K. A., Fama, J. M., Greenberg, J. L., \& Steketee, G. (2011). Modular cognitive behavioral therapy for body dysmorphic disorder. Behavior Therapy, 42, 624633. 
Williams, J., Hadjistavropoulos, T., \& Sharpe, D. (2006). A meta-analysis of psychological treatments for body dysmorphic disorder. Behavior Research and Therapy, 44, 99-111.

Wincze, J.P., Steketee, G., \& Frost, R.O. (2007). Categorization in compulsive hoarding. Behavior Research and Therapy, 45, 63-72.

Woods, D. W., Flessner, C. A., Franklin, M. E., Keuthen, N. J., Goodwin, R. D., Stein, D. J., et al. (2006). The Trichotillomania Impact Project (TIP): exploring phenomenology, functional impairment, and treatment utilization. Journal of Clinical Psychiatry, 67, 1877-1888.

Woody, S.R., Kellman-McFarlane, K., \& Welsted, A. (2014). Review of cognitive performance in hoarding disorder. Clinical Psychological Review, 34(4), 324-336.

Woody, S. R., Steketee, G., \& Chambless, D. L. (1995). Reliability and validity of the YaleBrown Obsessive-Compulsive Scale. Behavior Research and Therapy, 33, 597-605.

Woody, S. R., Whitall, M. L., \& McLean, P. D. (2011). Mechanisms of symptom reduction in the treatment of obsessions. Journal of Consulting and Clinical Psychology, 79, 653-664.

Woolley, J., Heyman, I., Brammer, M., Frampton, I., McGuire, P. K., \& Rubia, K. (2008). Brain activation in paediatric obsessive compulsive disorder during tasks of inhibitory control. British Journal of Psychiatry, 192, 25-31.

Wootton, B.M., Diefenbach, G.J., Bragdon, L.B., Steketee, G., Frost, R.O., \& Tolin, D.F. (2015). A contemporary psychometric evaluation of the obsessive compulsive inventoryrevised (OCI-R). Psychological Assessment, 27(3), 874-882.

Wright, L., Lipszyc, J., Dupuis, A., Thayapararajah, S.W., \& Schachar, R. (2014). Response inhibition and psychopathology: a meta-analysis of go/no-go task performance. Journal of Abnormal Psychology, 123(2), 429-439. 
Yook, K., Kim, K., Suh, S.Y., \& Lee, K.S. (2010). Intolerance of uncertainty, worry, and rumination in major depressive disorder and generalized anxiety disorder. Journal of Anxiety Disorders, 24(6), 623-628.

Zanarini, M.C., Skodol, A.E., Bender, D., Dolan, R., Sanislow, C., Schaefer, E., et al. (2000). The collaborative longitudinal personality disorders study: reliability of axis I and II diagnoses. Journal of Personality Disorders, 14(4), 291-299.

Zhang, Z., Fan, Q., Zhu, Y., Tan, L., Chen, Y., Gao, R., et al. (2017). Intrinsic functional connectivity alteration of dorsal and rostral anterior cingulate cortex in obsessivecompulsive disorder: a resting fMRI study. Neuroscience Letters, 654, 86-92.

Zhong, C.B., \& Liljenquist, K. (2006). Washing away your sins: threatened morality and physical cleansing. Science, 313, 1451-1452.

Zielinski, C. M., Taylor, M. A., \& Juzwin, K. R. (1991). Neuropsychological deficits in obsessive-compulsive disorder. Neuropsychiatry, Neuropsychology and Behavioral Neurology, 4, 110-126.

Zohar, J., \& the Cape Town Consensus Group (2006). Current controversies in the diagnosis and treatment of obsessive compulsive disorder- the Cape Town consensus statement. Biological Psychiatry, 59, 231.

Zohar, J., \& Insel, T. R. (1987). Drug treatment of obsessive-compulsive disorder. Journal of Affective Disorders, 13, 193-202. 
